\title{
Untapped Potential of Marine-Associated Cladosporium Species: An Overview on Secondary Metabolites, Biotechnological Relevance, and Biological Activities
}

\author{
Gamal A. Mohamed ${ }^{1, *}$ and Sabrin R. M. Ibrahim ${ }^{2,3} \mathbb{D}$ \\ 1 Department of Natural Products and Alternative Medicine, Faculty of Pharmacy, King Abdulaziz University, \\ Jeddah 21589, Saudi Arabia \\ 2 Preparatory Year Program, Batterjee Medical College, Jeddah 21442, Saudi Arabia; \\ sabrin.ibrahim@bmc.edu.sa \\ 3 Department of Pharmacognosy, Faculty of Pharmacy, Assiut University, Assiut 71526, Egypt \\ * Correspondence: gahussein@kau.edu.sa; Tel.: +966-597-636-182
}

Citation: Mohamed, G.A.; Ibrahim, S.R.M. Untapped Potential of Marine-Associated Cladosporium Species: An Overview on Secondary Metabolites, Biotechnological Relevance, and Biological Activities. Mar. Drugs 2021, 19, 645. https:// doi.org/10.3390/md19110645

Academic Editor: Ipek Kurtboke

Received: 21 October 2021

Accepted: 16 November 2021

Published: 18 November 2021

Publisher's Note: MDPI stays neutral with regard to jurisdictional claims in published maps and institutional affiliations.

Copyright: (c) 2021 by the authors. Licensee MDPI, Basel, Switzerland. This article is an open access article distributed under the terms and conditions of the Creative Commons Attribution (CC BY) license (https:/ / creativecommons.org/licenses/by/ $4.0 /)$.

\begin{abstract}
The marine environment is an underexplored treasure that hosts huge biodiversity of microorganisms. Marine-derived fungi are a rich source of novel metabolites with unique structural features, bioactivities, and biotechnological applications. Marine-associated Cladosporium species have attracted considerable interest because of their ability to produce a wide array of metabolites, including alkaloids, macrolides, diketopiperazines, pyrones, tetralones, sterols, phenolics, terpenes, lactones, and tetramic acid derivatives that possess versatile bioactivities. Moreover, they produce diverse enzymes with biotechnological and industrial relevance. This review gives an overview on the Cladosporium species derived from marine habitats, including their metabolites and bioactivities, as well as the industrial and biotechnological potential of these species. In the current review, 286 compounds have been listed based on the reported data from 1998 until July 2021. Moreover, more than 175 references have been cited.
\end{abstract}

Keywords: bioactivity; biotechnology; Cladosporium; Cladosporiaceae; marine fungi; metabolite

\section{Introduction}

The marine environment covers approximately 70\% of the Earth's surface and represents an enormous pool of biodiversity resources [1-3]. Marine microorganisms possess the potential for several biotechnological and industrial applications and play an important ecological role $[4,5]$. The last decades have witnessed numerous studies in the natural metabolites derived from marine creatures or their associated microorganisms [6-8]. Marine-derived fungi consist of a wide range of parasites, saprotrophs, symbionts, epiphytes, and endophytes $[9,10]$. They can be obtained from various marine samples such as algae, seagrasses, corals, sponges, ascidians, crustaceans, bivalves, fishes, and inorganic matter [11,12]. Jones et al. reported 530 marine taxa in 321 genera, which included 12 Basidiomycota (nine genera), 94 asexual morphs (61 genera), and 424 Ascomycota (251 genera) [13]. In 2011, the number of marine fungi was estimated to be 10,000 to 12,500 species based on substrates and geographical locations [14]. Currently, 1901 species have been listed on the marine fungi website, in 769 genera, 88 orders, 226 families, 22 classes, and seven phyla [15]. They are acknowledged as a rich source of novel metabolites with unique structural features, bioactivities, and biotechnological applications that attracted the attention of many biologists and chemists [16]. Cladosporium (Cladosporiaceae) is one of the largest genera of dematiaceous hyphomycetes [17]. Cladosporium species are frequent airborne molds, which can be isolated from almost every environment and geographic location, because their small conidia are easily dispersed [18-21]. C. herbarum, C. cladosporioides, and C. sphaerospermum are its three major species [22]. It comprises many important plant pathogens causing stem rots and leaf spots such as C. fulvum is the causal agent of tomato leaf mold [23,24]. Some 
species are also known as common contaminants in clinical laboratories and cause allergic lung diseases [25-28]. Some species have been reported as endophytes and possessed a positive influence, for example, C. sphaerospermum isolated from Glycine max roots which can promote its growth [29]. Several species were linked to allergic rhinitis and respiratory arrest in asthmatic patients, and some are described as a cause of opportunistic phaeohyphomycosis, including subcutaneous and deep infections in humans and animals [30,31]. Some species are fungicolous that possess a potential for biological control in agriculture and forestry [32,33]. Moreover, many Cladosporium species have the potential to be used in various industrial processes [34,35]. Marine-associated Cladosporium species have attracted considerable interest because of their ability to produce a wide array of metabolites, including macrolides, pyrones, phenolics, alkaloids, diketopiperazines, terpenes, sterols, quinones, lactones, and tetramic acid derivatives. These metabolites possess versatile bioactivities such as anticancer, antimicrobial, antiviral, insecticidal, antifouling, anti-malarial, anti-hyperlipidemic, and $\alpha$-glucosidase and protein tyrosine phosphatase inhibiton [36-42] . It has been shown that these species have significant impacts on biotechnology, ecosystems, and food production. They are a wealthy source of enzymes such as pectinases, agarases, carrageenases, xylanases, laccases, peroxidases, tannases, invertases, cellulases, and reductases that have wide biotechnological influences in developing eco-friendly technologies in the pulp and paper industry, food and feed industries, biomasses and contaminants bioremediation and biodegradation, and generation chemicals and liquid fuels $[11,12,43-50]$. The main goal of this review is the focus on the reported research in Cladosporium species derived from a marine habitat, including the structures and bioactivities of the reported metabolites, as well as the industrial and biotechnological potential of these species (Tables 1 and 2). This work covers the studies that have appeared in literature from 1998 until July 2021. The structures and bioactivities of reported metabolites from Cladosporium species have been highlighted. Furthermore, the biotechnological and industrial potential of Cladosporium species has been summarized. We hope that this work can provide knowledge that can help for the dereplication and bioactivities evaluation of these marine-associated Cladosporium species. The present data were collected through the search on the various databases, including Web of Knowledge, ScienceDirect, SCOPUS, Taylor \& Francis, Wiley Online Library, PubMed, JACS, Springer, and Google Scholar.

\section{Importance of Marine Associated Cladosporium Species}

Recently, cold-active microbial enzymes have attracted a great attention, and they are preferred to the thermophilic and mesophilic enzymes due to the reduction in the energy expenditure and costs of processing accompanied by industrial heating steps [51]. Many marine-associated Cladosporium species display noticeable enzyme production capacity. Many of these enzymes are exclusively produced at low temperature and high salt concentrations. Therefore, they play a substantial ecological role in lignin-cellulosic materials decomposition in the marine environment. Besides, these enzymes can be utilized in various biotechnological applications and allow the performance of industrial processes even in harsh conditions. In this review, the biotechnological and industrial relevance of Cladosporium species has been highlighted.

The polycyclic aromatic hydrocarbons (PAHs) are volatile pollutants that can cause various environmental pollutions such as oceanic and freshwater contamination, which can take place during storage, use, or transportation of crude oil and its products. PAHs inhalation or ingestion through contaminated food and airborne contaminants leads to serious health disorders such as endocrine disruption, cancer, and reproductive and birth problems [52]. Therefore, introducing marine-adapted microorganisms to increase the PAH-biodegradation rate is an important approach to reduce PAHs concentration in the contaminated regions. Investigation of the PAH biodegradation potential of various marine-derived fungi revealed that Cladosporium sp. CBMAI 1237 had a great potential for bioremediation and biodegradation of PAHs (e.g., anthracene, anthrone, anthraquinone, acenaphthene, phenanthrene, fluorene, pyrene fluoranthene, and nitropyrene) even in a non-marine environment [44]. 
Table 1. Secondary metabolites reported from marine associated Cladosporium species.

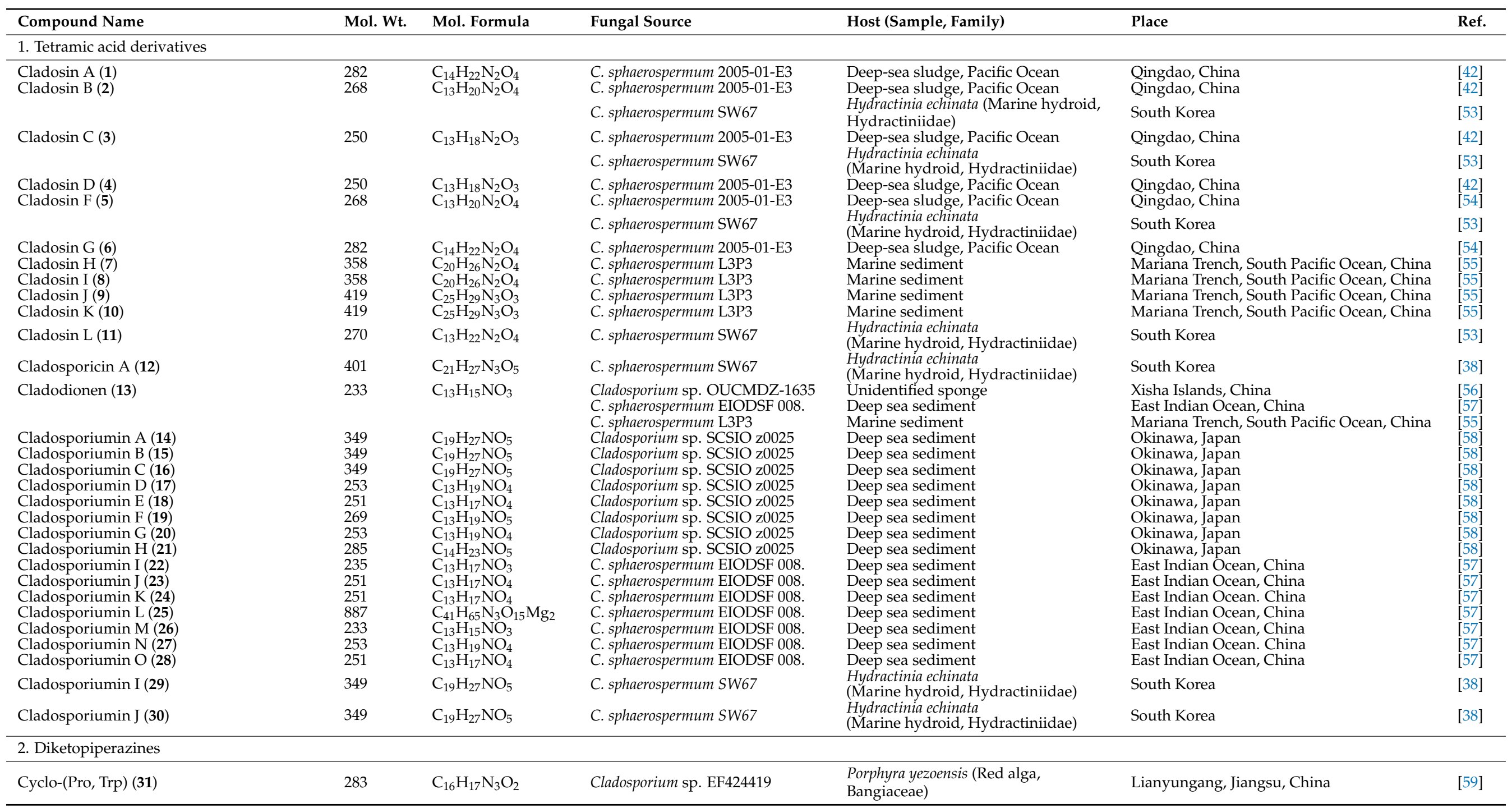


Table 1. Cont

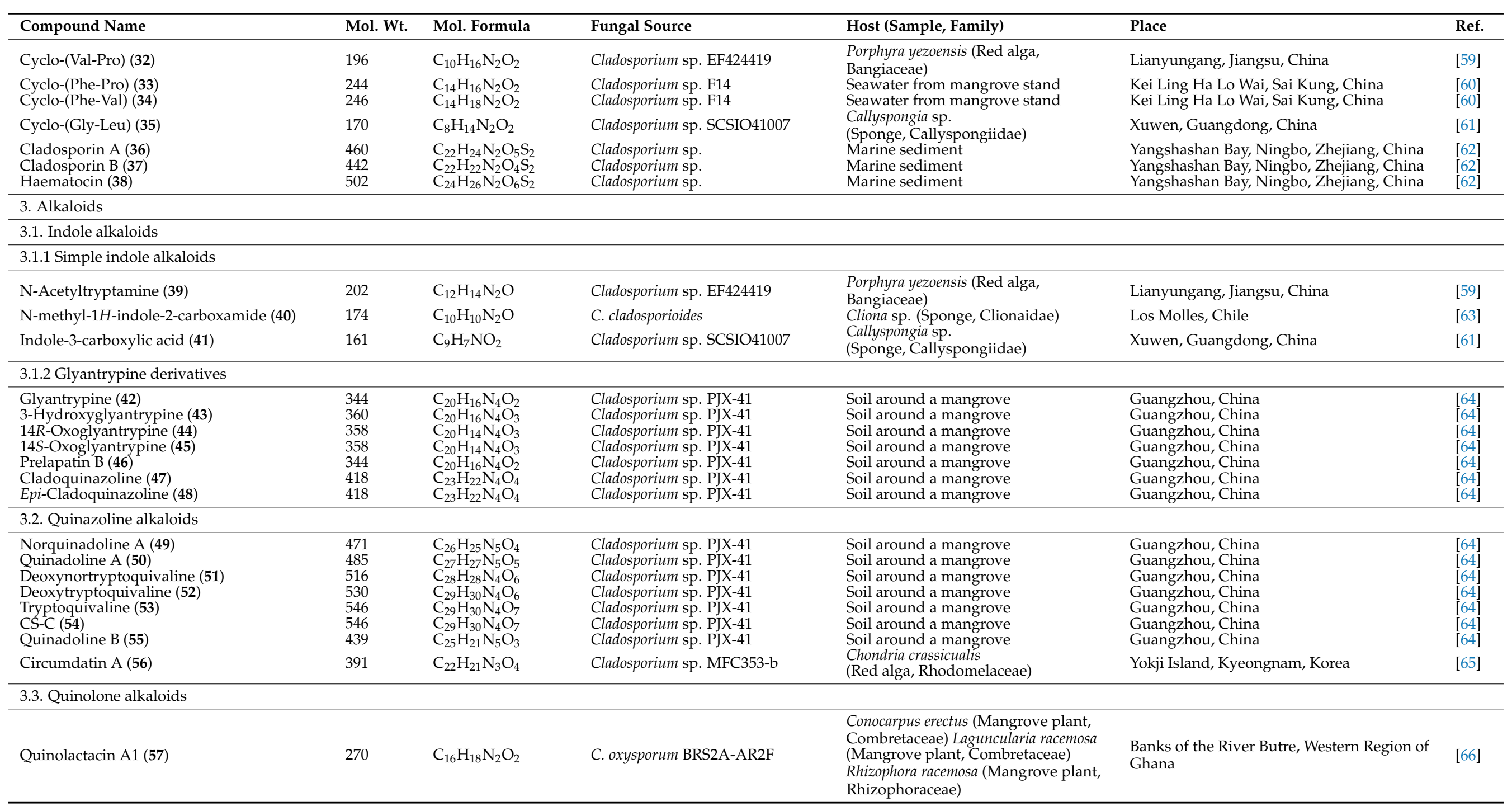


Table 1. Cont.

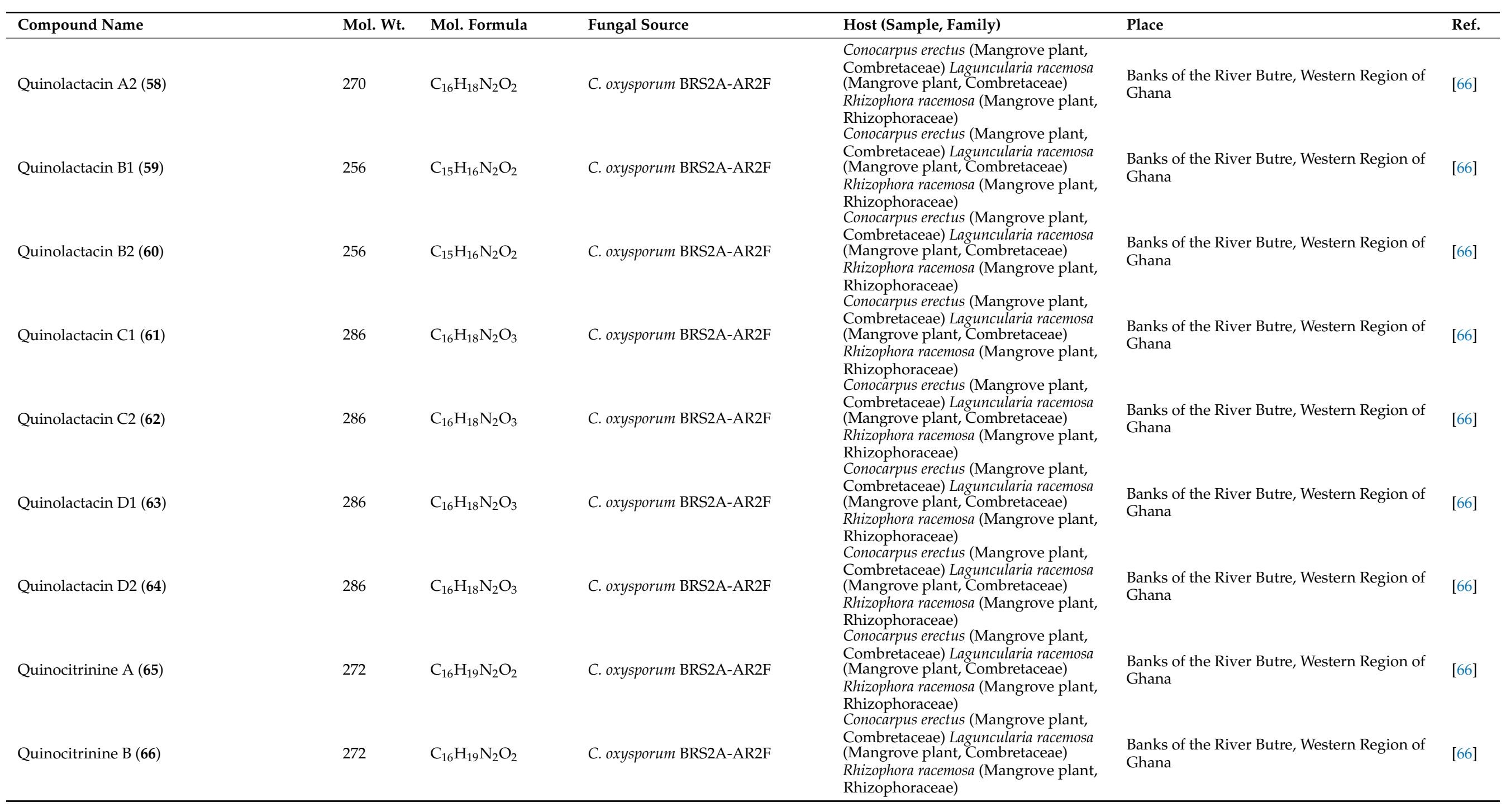


Table 1. Cont.

\begin{tabular}{|c|c|c|c|c|c|c|}
\hline Compound Name & Mol. Wt. & Mol. Formula & Fungal Source & Host (Sample, Family) & Place & Ref. \\
\hline Quinolactacide (67) & 236 & $\mathrm{C}_{14} \mathrm{H}_{8} \mathrm{~N}_{2} \mathrm{O}_{2}$ & C. oxysporum BRS2A-AR2F & $\begin{array}{l}\text { Conocarpus erectus (Mangrove plant, } \\
\text { Combretaceae) Laguncularia racemosa } \\
\text { (Mangrove plant, Combretaceae) } \\
\text { Rhizophora racemosa (Mangrove plant, } \\
\text { Rhizophoraceae) }\end{array}$ & $\begin{array}{l}\text { Banks of the River Butre, Western Region of } \\
\text { Ghana }\end{array}$ & [66] \\
\hline \multicolumn{7}{|l|}{ 3.4. Citrinadin derivatives } \\
\hline Citrinadin A (68) & 624 & $\mathrm{C}_{35} \mathrm{H}_{52} \mathrm{~N}_{4} \mathrm{O}_{6}$ & C. oxysporum BRS2A-AR2F & $\begin{array}{l}\text { Conocarpus erectus (Mangrove plant, } \\
\text { Combretaceae) Laguncularia racemosa } \\
\text { (Mangrove plant, Combretaceae) } \\
\text { Rhizophora racemosa (Mangrove plant, } \\
\text { Rhizophoraceae) }\end{array}$ & $\begin{array}{l}\text { Banks of the River Butre, Western Region of } \\
\text { Ghana }\end{array}$ & [66] \\
\hline Citrinadin B (69) & 481 & $\mathrm{C}_{28} \mathrm{H}_{39} \mathrm{~N}_{3} \mathrm{O}_{4}$ & C. oxysporum BRS2A-AR2F & $\begin{array}{l}\text { Conocarpus erectus (Mangrove plant, } \\
\text { Combretaceae) Laguncularia racemosa } \\
\text { (Mangrove plant, Combretaceae) } \\
\text { Rhizophora racemosa (Mangrove plant, } \\
\text { Rhizophoraceae) }\end{array}$ & $\begin{array}{l}\text { Banks of the River Butre, Western Region of } \\
\text { Ghana }\end{array}$ & [66] \\
\hline Butrecitrinadin (70) & 682 & $\mathrm{C}_{38} \mathrm{H}_{57} \mathrm{~N}_{4} \mathrm{O}_{7}$ & C. oxysporum BRS2A-AR2F & $\begin{array}{l}\text { Conocarpus erectus (Mangrove plant, } \\
\text { Combretaceae) Laguncularia racemosa } \\
\text { (Mangrove plant, Combretaceae) } \\
\text { Rhizophora racemosa (Mangrove plant, } \\
\text { Rhizophoraceae) }\end{array}$ & $\begin{array}{l}\text { Banks of the River Butre, Western Region of } \\
\text { Ghana }\end{array}$ & [66] \\
\hline PF1270 A (71) & 566 & $\mathrm{C}_{32} \mathrm{H}_{43} \mathrm{~N}_{3} \mathrm{O}_{6}$ & C. oxysporum BRS2A-AR2F & $\begin{array}{l}\text { Conocarpus erectus (Mangrove plant, } \\
\text { Combretaceae) Laguncularia racemosa } \\
\text { (Mangrove plant, Combretaceae) } \\
\text { Rhizophora racemosa (Mangrove plant, } \\
\text { Rhizophoraceae) }\end{array}$ & $\begin{array}{l}\text { Banks of the River Butre, Western Region of } \\
\text { Ghana }\end{array}$ & [66] \\
\hline PF1270 B (72) & 552 & $\mathrm{C}_{31} \mathrm{H}_{41} \mathrm{~N}_{3} \mathrm{O}_{6}$ & C. oxysporum BRS2A-AR2F & $\begin{array}{l}\text { Conocarpus erectus (Mangrove plant, } \\
\text { Combretaceae) Laguncularia racemosa } \\
\text { (Mangrove plant, Combretaceae) } \\
\text { Rhizophora racemosa (Mangrove plant, } \\
\text { Rhizophoraceae) }\end{array}$ & $\begin{array}{l}\text { Banks of the River Butre, Western Region of } \\
\text { Ghana }\end{array}$ & [66] \\
\hline PF1270 C (73) & 538 & $\mathrm{C}_{30} \mathrm{H}_{39} \mathrm{~N}_{3} \mathrm{O}_{6}$ & C. oxysporum BRS2A-AR2F & $\begin{array}{l}\text { Conocarpus erectus (Mangrove plant, } \\
\text { Combretaceae) Laguncularia racemosa } \\
\text { (Mangrove plant, Combretaceae) } \\
\text { Rhizophora racemosa (Mangrove plant, } \\
\text { Rhizophoraceae) }\end{array}$ & $\begin{array}{l}\text { Banks of the River Butre, Western Region of } \\
\text { Ghana }\end{array}$ & [66] \\
\hline \multicolumn{7}{|l|}{ 3.5. Pyrrolidine derivatives } \\
\hline Cladosporitin A (74) & 505 & $\mathrm{C}_{32} \mathrm{H}_{43} \mathrm{NO}_{4}$ & Cladosporium sp. HNWSW-1 & \multirow{4}{*}{$\begin{array}{l}\text { Ceriops tagal } \\
\text { (Mangrove plant, Rhizophoraceae) } \\
\text { Ceriops tagal } \\
\text { (Mangrove plant, Rhizophoraceae) } \\
\text { Ceriops tagal } \\
\text { (Mangrove plant, Rhizophoraceae) } \\
\text { Unidentified marine sponge }\end{array}$} & Dong Zhai Gang, Hainan, China & [67] \\
\hline Cladosporitin B (75) & 505 & $\mathrm{C}_{32} \mathrm{H}_{43} \mathrm{NO}_{4}$ & Cladosporium sp. HNWSW-1 & & Dong Zhai Gang, Hainan, China & [67] \\
\hline Talaroconvolutin A (76) & 487 & $\mathrm{C}_{32} \mathrm{H}_{41} \mathrm{NO}_{3}$ & Cladosporium sp. HNWSW-1 & & Dong Zhai Gang, Hainan, China & [67] \\
\hline Cladosporamide A (77) & 273 & $\mathrm{C}_{14} \mathrm{H}_{11} \mathrm{NO}_{5}$ & Cladosporium sp. TPU1507 & & Manado, Indonesia & [68] \\
\hline
\end{tabular}


Table 1. Cont

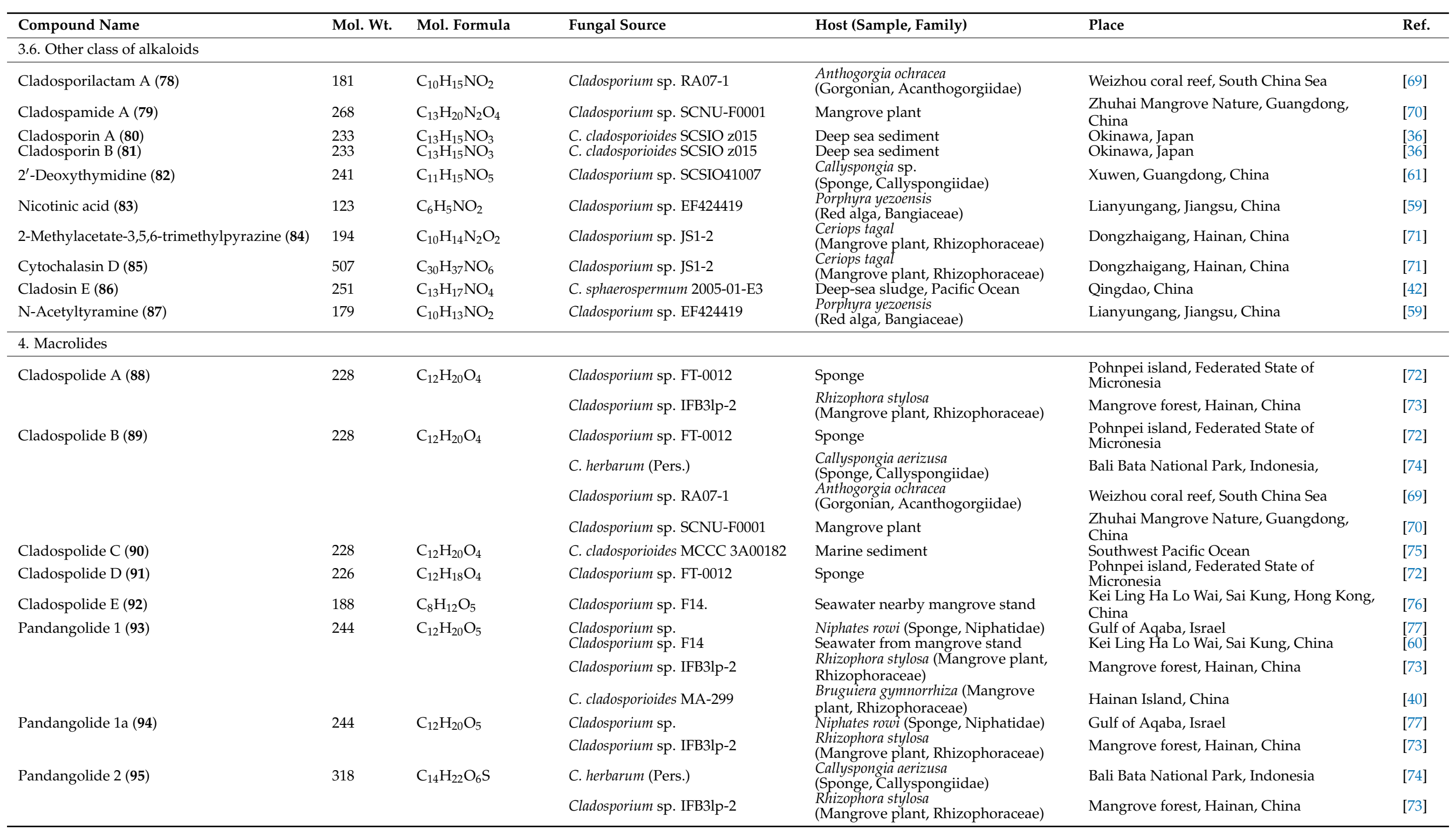


Table 1. Cont.

\begin{tabular}{|c|c|c|c|c|c|c|}
\hline Compound Name & Mol. Wt. & Mol. Formula & Fungal Source & Host (Sample, Family) & Place & Ref \\
\hline \multirow[t]{3}{*}{ Pandangolide 3 (96) } & 362 & $\mathrm{C}_{16} \mathrm{H}_{26} \mathrm{O}_{7} \mathrm{~S}$ & C. herbarum (Pers.) & $\begin{array}{l}\text { Callyspongia aerizusa } \\
\text { (Sponge, Callyspongiidae) }\end{array}$ & Bali Bata National Park, Indonesia, & {$[74]$} \\
\hline & & & Cladosporium sp. IFB3lp-2 & $\begin{array}{l}\text { Rhizophora stylosa } \\
\text { (Mangrove plant, Rhizophoraceae) }\end{array}$ & Mangrove forest, Hainan, China & {$[73]$} \\
\hline & & & C. oxysporum HDN13-314 & $\begin{array}{l}\text { Avicennia marina } \\
\text { (Mangrove plant, Acanthaceae) }\end{array}$ & Hainan, China & {$[78]$} \\
\hline Pandangolide 4 (97) & 486 & $\mathrm{C}_{24} \mathrm{H}_{38} \mathrm{O}_{8} \mathrm{~S}$ & C. herbarum (Pers.) & $\begin{array}{l}\text { Callyspongia aerizusa } \\
\text { (Sponge, Callyspongiidae) }\end{array}$ & Bali Bata National Park, Indonesia & {$[74]$} \\
\hline $5 R$-Hydroxyrecifeiolide (98) & 212 & $\mathrm{C}_{12} \mathrm{H}_{20} \mathrm{O}_{3}$ & C. cladosporioides MA-299 & $\begin{array}{l}\text { Bruguiera gymnorrhiza } \\
\text { (Mangrove plant, Rhizophoraceae) }\end{array}$ & Hainan Island, China & {$[40]$} \\
\hline 5S-Hydroxyrecifeiolide (99) & 212 & $\mathrm{C}_{12} \mathrm{H}_{20} \mathrm{O}_{3}$ & C. cladosporioides MA-299 & $\begin{array}{l}\text { Bruguiera gymnorrhiza } \\
\text { (Mangrove plant, Rhizophoraceae) }\end{array}$ & Hainan Island, China & {$[40]$} \\
\hline $\begin{array}{l}\text { Methyl 2-(((4R,6R,12R)-6-hydroxy-12- } \\
\text { methyl-2,5-dioxooxacyclodo } \\
\text { decan-4-yl)thio)acetate (100) }\end{array}$ & 332 & $\mathrm{C}_{15} \mathrm{H}_{24} \mathrm{O}_{6} \mathrm{~S}$ & Cladosporium sp. IFB3lp-2 & $\begin{array}{l}\text { Rhizophora stylosa } \\
\text { (Mangrove plant, Rhizophoraceae) }\end{array}$ & Mangrove forest, Hainan, China & {$[73]$} \\
\hline \multirow[t]{2}{*}{ Thiocladospolide A (101) } & 346 & $\mathrm{C}_{16} \mathrm{H}_{26} \mathrm{O}_{6} \mathrm{~S}$ & C. cladosporioides MA-299 & $\begin{array}{l}\text { Bruguiera gymnorrhiza } \\
\text { (Mangrove plant, Rhizophoraceae) }\end{array}$ & Hainan Island, China & {$[39]$} \\
\hline & & & C. oxysporum HDN13-314 & $\begin{array}{l}\text { Avicennia marina } \\
\text { (Mangrove plant, Acanthaceae) }\end{array}$ & Hainan, China & {$[78]$} \\
\hline Thiocladospolide B (102) & 360 & $\mathrm{C}_{16} \mathrm{H}_{24} \mathrm{O}_{7} \mathrm{~S}$ & C. cladosporioides MA-299 & $\begin{array}{l}\text { Bruguiera gymnorrhiza } \\
\text { (Mangrove plant, Rhizophoraceae) }\end{array}$ & Hainan Island, China & [39] \\
\hline Thiocladospolide C (103) & 330 & $\mathrm{C}_{15} \mathrm{H}_{22} \mathrm{O}_{6} \mathrm{~S}$ & C. cladosporioides MA-299 & $\begin{array}{l}\text { Bruguiera gymnorrhiza } \\
\text { (Mangrove plant, Rhizophoraceae) }\end{array}$ & Hainan Island, China & [39] \\
\hline Thiocladospolide E (105) & 306 & $\mathrm{C}_{14} \mathrm{H}_{26} \mathrm{O}_{5} \mathrm{~S}$ & Cladosporium sp. SCNU-F0001 & Mangrove plant & $\begin{array}{l}\text { Zhuhai Mangrove Nature, Guangdong, } \\
\text { China }\end{array}$ & {$[70]$} \\
\hline Thiocladospolide F (106) & 332 & $\mathrm{C}_{16} \mathrm{H}_{28} \mathrm{O}_{5} \mathrm{~S}$ & C. cladosporioides MA-299 & $\begin{array}{l}\text { Bruguiera gymnorrhiza } \\
\text { (Mangrove plant, Rhizophoraceae) }\end{array}$ & Hainan Island, China & [79] \\
\hline Thiocladospolide F (107) & 386 & $\mathrm{C}_{24} \mathrm{H}_{38} \mathrm{O}_{8} \mathrm{~S}$ & C. oxysporum HDN13-314 & $\begin{array}{l}\text { Avicennia marina } \\
\text { (Mangrove plant, Acanthaceae) }\end{array}$ & Hainan, China & [78] \\
\hline Thiocladospolide G (108) & 348 & $\mathrm{C}_{16} \mathrm{H}_{28} \mathrm{O}_{6} \mathrm{~S}$ & C. cladosporioides MA-299 & $\begin{array}{l}\text { Bruguiera gymnorrhiza } \\
\text { (Mangrove plant, Rhizophoraceae) }\end{array}$ & Hainan Island, China & {$[79]$} \\
\hline Thiocladospolide G (109) & 348 & $\mathrm{C}_{15} \mathrm{H}_{24} \mathrm{O}_{7} \mathrm{~S}$ & C. oxysporum HDN13-314 & $\begin{array}{l}\text { Avicennia marina } \\
\text { (Mangrove plant, Acanthaceae) }\end{array}$ & Hainan, China & [78] \\
\hline Thiocladospolide H (110) & 332 & $\mathrm{C}_{15} \mathrm{H}_{24} \mathrm{O}_{6} \mathrm{~S}$ & C. oxysporum HDN13-314 & $\begin{array}{l}\text { Avicennia marina } \\
\text { (Mangrove plant, Acanthaceae) }\end{array}$ & Hainan, China & {$[78]$} \\
\hline Thiocladospolide I (111) & 560 & $\mathrm{C}_{27} \mathrm{H}_{44} \mathrm{O}_{10} \mathrm{~S}$ & C. oxysporum HDN13-314 & $\begin{array}{l}\text { Avicennia marina } \\
\text { (Mangrove plant, Acanthaceae) }\end{array}$ & Hainan, China & [78] \\
\hline Thiocladospolide J (112) & 558 & $\mathrm{C}_{27} \mathrm{H}_{42} \mathrm{O}_{10} \mathrm{~S}$ & C. oxysporum HDN13-314 & $\begin{array}{l}\text { Avicennia marina } \\
\text { (Mangrove plant, Acanthaceae) }\end{array}$ & Hainan, China & {$[78]$} \\
\hline Sporiolide A (113) & 348 & $\mathrm{C}_{19} \mathrm{H}_{24} \mathrm{O}_{6}$ & Cladosporium sp. L037 & $\begin{array}{l}\text { Actinotrichia fragilis } \\
\text { (Red alga, Galaxauraceae) }\end{array}$ & Seragaki Beach, Okinawa Island, Japan & {$[80]$} \\
\hline Sporiolide B (114) & 258 & $\mathrm{C}_{13} \mathrm{H}_{22} \mathrm{O}_{5}$ & Cladosporium sp. L037 & $\begin{array}{l}\text { Actinotrichia fragilis } \\
\text { (Red alga, Galaxauraceae) }\end{array}$ & Seragaki Beach, Okinawa Island, Japan & {$[80]$} \\
\hline $\begin{array}{l}(6 R, 12 S)-6-H y d r o x y-12-m e t h y l-1- \\
\text { oxacyclododecane-2,5-dione } \\
(\mathbf{1 1 5})\end{array}$ & 228 & $\mathrm{C}_{12} \mathrm{H}_{20} \mathrm{O}_{4}$ & Cladosporium sp. F14 & Seawater from the mangrove stand & Kei Ling Ha Lo Wai, Sai Kung, China & {$[60]$} \\
\hline
\end{tabular}


Table 1. Cont.

\begin{tabular}{|c|c|c|c|c|c|c|}
\hline Compound Name & Mol. Wt. & Mol. Formula & Fungal Source & Host (Sample, Family) & Place & Ref. \\
\hline $\begin{array}{l}\text { (3R,6S)-6-Hydroxy-12-methyl-2,5- } \\
\text { dioxooxacyclododecan-3-yl } \\
\text { (E)-4,11-dihydroxydodec-2-enoate (116) }\end{array}$ & 456 & $\mathrm{C}_{24} \mathrm{H}_{40} \mathrm{O}_{8}$ & Cladosporium sp. IFB3lp-2 & $\begin{array}{l}\text { Rhizophora stylosa } \\
\text { (Mangrove plant, Rhizophoraceae) }\end{array}$ & Mangrove forest, Hainan, China & [73] \\
\hline Dendrodolide A (117) & 256 & $\mathrm{C}_{13} \mathrm{H}_{20} \mathrm{O}_{5}$ & Cladosporium sp. RA07-1 & \multirow{4}{*}{$\begin{array}{l}\text { Anthogorgia ochracea } \\
\text { (Gorgonian, Acanthogorgiidae) } \\
\text { Anthogorgia ochracea } \\
\text { (Gorgonian, Acanthogorgiidae) } \\
\text { Anthogorgia ochracea } \\
\text { (Gorgonian, Acanthogorgiidae) } \\
\text { Anthogorgia ochracea } \\
\text { (Gorgonian, Acanthogorgiidae) } \\
\text { Bruguiera gymnorrhiza } \\
\text { (Mangrove plant, Rhizophoraceae) }\end{array}$} & Weizhou coral reef, South China Sea & [69] \\
\hline Dendrodolide C (118) & 242 & $\mathrm{C}_{12} \mathrm{H}_{18} \mathrm{O}_{5}$ & Cladosporium sp. RA07-1 & & Weizhou coral reef, South China Sea & [69] \\
\hline Dendrodolide L (119) & 228 & $\mathrm{C}_{12} \mathrm{H}_{20} \mathrm{O}_{4}$ & Cladosporium sp. RA07-1 & & Weizhou coral reef, South China Sea & [69] \\
\hline Dendrodolide M (120) & 256 & $\mathrm{C}_{13} \mathrm{H}_{20} \mathrm{O}_{5}$ & Cladosporium sp. RA07-1 & & Weizhou coral reef, South China Sea & [69] \\
\hline \multicolumn{7}{|l|}{ 5. Butenolides and butanolides } \\
\hline Cladospolide F (122) & 230 & $\mathrm{C}_{12} \mathrm{H}_{22} \mathrm{O}_{4}$ & Cladosporium sp. TZP29 & \multirow{11}{*}{$\begin{array}{l}\text { Unidentified soft coral } \\
\text { Bruguiera gymnorrhiza } \\
\text { (Mangrove plant, Rhizophoraceae) } \\
\text { Bruguiera gymnorrhiza } \\
\text { (Mangrove plant, Rhizophoraceae) } \\
\text { Unidentified soft coral } \\
\text { Callyspongia aerizusa } \\
\text { (Sponge, Callyspongiidae) } \\
\text { Niphates rowi (Sponge, Niphatidae) } \\
\text { Seawater from the mangrove stand } \\
\text { Rhizophora stylosa } \\
\text { (Mangrove plant, Rhizophoraceae) } \\
\text { Anthogorgia ochracea } \\
\text { (Gorgonian, Acanthogorgiidae) } \\
\text { Unidentified soft coral } \\
\text { Bruguiera gymnorrhiza } \\
\text { (Mangrove plant, Rhizophoraceae) } \\
\text { Avicennia marina } \\
\text { (Mangrove plant, Acanthaceae) } \\
\text { Bruguiera gymnorrhiza } \\
\text { (Mangrove plant, Rhizophoraceae) }\end{array}$} & Guangzhou, China & [41] \\
\hline Ent-cladospolide F (123) & 230 & $\mathrm{C}_{14} \mathrm{H}_{24} \mathrm{O}_{5}$ & C. cladosporioides MA-299 & & Hainan Island, China & [40] \\
\hline Cladospolide G (124) & 272 & $\mathrm{C}_{14} \mathrm{H}_{24} \mathrm{O}_{5}$ & C. cladosporioides MA-299 & & Hainan Island, China & [40] \\
\hline 11-Hydroxy- $\gamma$-dodecalactone (125) & 214 & $\mathrm{C}_{12} \mathrm{H}_{22} \mathrm{O}_{3}$ & Cladosporium sp. TZP29 & & Guangzhou, China & [41] \\
\hline Iso-Cladospolide B (126) & 228 & $\mathrm{C}_{12} \mathrm{H}_{20} \mathrm{O}_{4}$ & C. herbarum (Pers.) & & Bali Bata National Park, Indonesia, & [74] \\
\hline & & & $\begin{array}{l}\text { Cladosporium sp. } \\
\text { Cladosporium sp. F14 }\end{array}$ & & $\begin{array}{l}\text { Gulf of Aqaba, Israel } \\
\text { Kei Ling Ha Lo Wai, Sai Kung, China }\end{array}$ & {$[77]$} \\
\hline & & & Cladosporium sp. RA07-1 & & Weizhou coral reef, South China Sea & [70] \\
\hline & & & Cladosporium sp. TZP29 & & Guangzhou, China & [41] \\
\hline & & & C. cladosporioides MA-299 & & Hainan Island, China & [40] \\
\hline & & & C. oxysporum HDN13-314 & & Hainan, China & {$[78]$} \\
\hline Cladospolide H (127) & 210 & $\mathrm{C}_{12} \mathrm{H}_{18} \mathrm{O}_{3}$ & C. cladosporioides MA-299 & & Hainan Island, China & [40] \\
\hline \multicolumn{7}{|l|}{ 6. Seco-acids } \\
\hline Cladospolide A II (128) & & & Cladosporium sp. IFB3lp-2 & \multirow{5}{*}{$\begin{array}{l}\text { Rhizophora stylosa } \\
\text { (Mangrove plant, Rhizophoraceae) } \\
\text { Unidentified soft coral } \\
\text { Unidentified soft coral } \\
\text { Seawater from the Mangrove stand } \\
\text { Unidentified soft coral } \\
\text { Bruguiera gymnorrhiza } \\
\text { (Mangrove plant, Rhizophoraceae) } \\
\text { Avicennia marina } \\
\text { (Mangrove plant, Acanthaceae) } \\
\text { Rhizophora stylosa } \\
\text { (Mangrove plant, Rhizophoraceae) }\end{array}$} & Mangrove forest, Hainan, China & [73] \\
\hline $\begin{array}{l}\text { Cladospolide E (129) } \\
\text { Seco-Patulolide A (130) } \\
\text { Seco-Patulolide C (131) }\end{array}$ & $\begin{array}{l}228 \\
228 \\
230\end{array}$ & $\begin{array}{l}\mathrm{C}_{12} \mathrm{H}_{20} \mathrm{O}_{4} \\
\mathrm{C}_{12} \mathrm{H}_{20} \mathrm{O}_{4} \\
\mathrm{C}_{12} \mathrm{H}_{22} \mathrm{O}_{4}\end{array}$ & $\begin{array}{l}\text { Cladosporium sp. TZP29 } \\
\text { Cladosporium sp. TZP29 } \\
\text { Cladosporium sp. F14 } \\
\text { Cladosporium sp. TZP29 }\end{array}$ & & $\begin{array}{l}\text { Guangzhou, China } \\
\text { Guangzhou, China } \\
\text { Kei Ling Ha Lo Wai, Sai Kung, China } \\
\text { Guangzhou, China }\end{array}$ & $\begin{array}{l}{[41]} \\
{[41]} \\
{[60]} \\
{[41]}\end{array}$ \\
\hline & & & C. cladosporioides MA-299 & & Hainan Island, China & [39] \\
\hline Seco-Secopatulolide C (132) & 230 & $\mathrm{C}_{12} \mathrm{H}_{22} \mathrm{O}_{4}$ & C. oxysporum HDN13-314 & & Hainan, China & {$[78]$} \\
\hline Cladosporester A (133) & 244 & $\mathrm{C}_{13} \mathrm{H}_{24} \mathrm{O}_{4}$ & C. cladosporioides OUCMDZ-187 & & Shankou, Guangxi, China & [81] \\
\hline
\end{tabular}


Table 1. Cont.

\begin{tabular}{|c|c|c|c|c|c|c|}
\hline Compound Name & Mol. Wt. & Mol. Formula & Fungal Source & Host (Sample, Family) & Place & Ref. \\
\hline Cladosporester B (134) & 244 & $\mathrm{C}_{13} \mathrm{H}_{24} \mathrm{O}_{4}$ & C. cladosporioides OUCMDZ-187 & \multirow{7}{*}{$\begin{array}{l}\text { Rhizophora stylosa } \\
\text { (Mangrove plant, Rhizophoraceae) } \\
\text { Rhizophora stylosa } \\
\text { (Mangrove plant, Rhizophoraceae) } \\
\text { Rhizophora stylosa } \\
\text { (Mangrove plant, Rhizophoraceae) } \\
\text { Rhizophora stylosa } \\
\text { (Mangrove plant, Rhizophoraceae) } \\
\text { Rhizophora stylosa } \\
\text { (Mangrove plant, Rhizophoraceae) } \\
\text { Rhizophora stylosa } \\
\text { (Mangrove plant, Rhizophoraceae) } \\
\text { Rhizophora stylosa } \\
\text { (Mangrove plant, Rhizophoraceae) } \\
\text { Rhizophora stylosa } \\
\text { (Mangrove plant, Rhizophoraceae) }\end{array}$} & Shankou, Guangxi, China & [81] \\
\hline Cladosporacid A (135) & 230 & $\mathrm{C}_{12} \mathrm{H}_{22} \mathrm{O}_{4}$ & C. cladosporioides OUCMDZ-187 & & Shankou, Guangxi, China & [81] \\
\hline Cladosporacid D (137) & 228 & $\mathrm{C}_{12} \mathrm{H}_{20} \mathrm{O}_{4}$ & C. cladosporioides OUCMDZ-187 & & Shankou, Guangxi, China & [81] \\
\hline Cladosporester C (138) & 288 & $\mathrm{C}_{14} \mathrm{H}_{24} \mathrm{O}_{6}$ & C. cladosporioides OUCMDZ-187 & & Shankou, Guangxi, China & [81] \\
\hline Cladosporacid C (139) & 230 & $\mathrm{C}_{12} \mathrm{H}_{22} \mathrm{O}_{4}$ & C. cladosporioides OUCMDZ-187 & & Shankou, Guangxi, China & [81] \\
\hline Cladosporacid E (140) & 200 & $\mathrm{C}_{10} \mathrm{H}_{16} \mathrm{O}_{4}$ & C. cladosporioides OUCMDZ-187 & & Shankou, Guangxi, China & [81] \\
\hline 11-Hydroxy-4,5-dioxododecanoic acid (141) & 244 & $\mathrm{C}_{10} \mathrm{H}_{16} \mathrm{O}_{4}$ & Cladosporium sp. IFB3lp-2 & & Mangrove forest, Hainan, China & [73] \\
\hline \multicolumn{7}{|l|}{ 7. Tetralones (napthalenones) } \\
\hline Cladosporol/Cladosporol A (142) & 352 & $\mathrm{C}_{20} \mathrm{H}_{16} \mathrm{O}_{6}$ & Cladosporium sp. KcFL6' & \multirow{18}{*}{$\begin{array}{l}\text { Kandelia candel } \\
\text { (Mangrove plant, Rhizophoraceae) } \\
\text { Kandelia candel } \\
\text { (Mangrove plant, Rhizophoraceae) } \\
\text { Marine sediment } \\
\text { Laurencia okamurai } \\
\text { (Red alga, Rhodomelaceae) } \\
\text { Ceriops tagal } \\
\text { (Mangrove plant, Rhizophoraceae) } \\
\text { Marine sediment } \\
\text { Kandelia candel } \\
\text { (Mangrove plant, Rhizophoraceae) } \\
\text { Marine sediment } \\
\text { Ceriops tagal } \\
\text { (Mangrove plant, Rhizophoraceae) } \\
\text { Marine sediment } \\
\text { Laurencia okamurai } \\
\text { (Red alga, Rhodomelaceae) } \\
\text { Marine sediment } \\
\text { Laurencia okamurai } \\
\text { (Red alga, Rhodomelaceae) } \\
\text { Laurencia okamurai } \\
\text { (Red alga, Rhodomelaceae) } \\
\text { Laurencia okamurai } \\
\text { (Rhodomelaceae) } \\
\text { Blood cockle } \\
\text { (Bivalve mollusk, Cardiidae) } \\
\text { Laurencia okamurai } \\
\text { (Red alga, Rhodomelaceae) } \\
\text { Kandelia candel } \\
\text { (Mangrove plant, Rhizophoraceae) }\end{array}$} & Daya Bay, Shenzhen city, Guangdong, China & [82] \\
\hline Cladosporol C (143) & 338 & $\mathrm{C}_{20} \mathrm{H}_{18} \mathrm{O}_{5}$ & Cladosporium sp. KcFL6' & & Daya Bay, Shenzhen city, Guangdong, China & [82] \\
\hline & & & C. cladosporioides HDN14-342 & & Indian Ocean, Qingdao, China & [83] \\
\hline & & & C. cladosporioides EN-399 & & Qingdao, China & [84] \\
\hline & & & C. cladosporioides MCCC 3A00182 & & Southwest Pacific Ocean & [75] \\
\hline Cladosporol D (144) & 354 & $\mathrm{C}_{20} \mathrm{H}_{18} \mathrm{O}_{6}$ & Cladosporium sp. KcFL6' & & Daya Bay, Shenzhen city, Guangdong, China & [82] \\
\hline Cladosporol E (145) & 370 & $\mathrm{C}_{20} \mathrm{H}_{18} \mathrm{O}_{7}$ & C. cladosporioides HDN14-342 & & Indian Ocean, Qingdao, China & [83] \\
\hline & & & Cladosporium sp. JS1-2 & & Dongzhaigang, Hainan, China & [71] \\
\hline Cladosporol F (146) & 352 & $\mathrm{C}_{21} \mathrm{H}_{20} \mathrm{O}_{5}$ & C. cladosporioides HDN14-342 & & Indian Ocean, Qingdao, China & [83] \\
\hline & & & C. cladosporioides EN-399 & & Qingdao, China & [84] \\
\hline Cladosporol G (147) & 388 & $\mathrm{C}_{20} \mathrm{H}_{17} \mathrm{ClO}_{6}$ & C. cladosporioides HDN14-342 & & Indian Ocean, Qingdao, China & [83] \\
\hline Cladosporol G (148) & 352 & $\mathrm{C}_{21} \mathrm{H}_{20} \mathrm{O}_{5}$ & C. cladosporioides EN-399 & & Qingdao, China & [84] \\
\hline Cladosporol H (149) & 336 & $\mathrm{C}_{20} \mathrm{H}_{16} \mathrm{O}_{5}$ & C. cladosporioides EN-399 & & Qingdao, China & [84] \\
\hline \multirow{3}{*}{ Cladosporol I = Cladosperanol A (150) } & 338 & $\mathrm{C}_{20} \mathrm{H}_{18} \mathrm{O}_{5}$ & C. cladosporioides EN-399 & & Qingdao, China & [84] \\
\hline & & & Cladosporium sp. KFD33 & & Haikou Bay, China & [85] \\
\hline & 338 & $\mathrm{C}_{20} \mathrm{H}_{18} \mathrm{O}_{5}$ & C. perangustum FS62 & & China & [86] \\
\hline Cladosporol J (151) & 338 & $\mathrm{C}_{20} \mathrm{H}_{18} \mathrm{O}_{5}$ & C. cladosporioides EN-399 & & Qingdao, China & [84] \\
\hline Cladosporone A (152) & 352 & $\mathrm{C}_{20} \mathrm{H}_{16} \mathrm{O}_{6}$ & Cladosporium sp. KcFL6' & & Daya Bay, Shenzhen city, Guangdong, China & [82] \\
\hline
\end{tabular}


Table 1. Cont.

\begin{tabular}{|c|c|c|c|c|c|c|}
\hline Compound Name & Mol. Wt. & Mol. Formula & Fungal Source & Host (Sample, Family) & Place & Ref \\
\hline Altertoxin XII (153) & 322 & $\mathrm{C}_{20} \mathrm{H}_{18} \mathrm{O}_{4}$ & Cladosporium sp. KFD33 & \multirow{3}{*}{$\begin{array}{l}\text { Blood cockle } \\
\text { (Bivalve mollusk, Cardiidae) } \\
\text { Marine sediment } \\
\text { Marine sediment } \\
\text { Marine sediment } \\
\text { Marine sediment } \\
\text { Ceriops tagal } \\
\text { (Mangrove plant, Rhizophoraceae) }\end{array}$} & Haikou Bay, China & {$[85]$} \\
\hline $\begin{array}{l}\text { Clindanone A (154) } \\
\text { Clindanone B (155) } \\
\text { Isosclerone = (-)-(4R)-Regiolone (156) }\end{array}$ & $\begin{array}{l}394 \\
394 \\
178 \\
178\end{array}$ & $\begin{array}{l}\mathrm{C}_{22} \mathrm{H}_{18} \mathrm{O}_{7} \\
\mathrm{C}_{22} \mathrm{H}_{18} \mathrm{O}_{7} \\
\mathrm{C}_{10} \mathrm{H}_{10} \mathrm{O}_{3} \\
\mathrm{C}_{10} \mathrm{H}_{10} \mathrm{O}_{3}\end{array}$ & $\begin{array}{l}\text { C. cladosporioides HDN14-342 } \\
\text { C. cladosporioides HDN14-342 } \\
\text { C. perangustm FS62 } \\
\text { C. cladosporioides HDN14-342 }\end{array}$ & & \multirow{2}{*}{$\begin{array}{l}\text { Indian Ocean, Qingdao, China } \\
\text { Indian Ocean, Qingdao, China } \\
\text { South China Sea, china } \\
\text { Indian Ocean, Qingdao, China } \\
\text { South China Sea, Dongzhaigang, Hainan, } \\
\text { China }\end{array}$} & $\begin{array}{l}{[83]} \\
{[83]} \\
{[87]} \\
{[83]}\end{array}$ \\
\hline Isosclerone $=(-)-(4 R)-$ Regiolone $(\mathbf{1 5 6})$ & 178 & $\mathrm{C}_{10} \mathrm{H}_{10} \mathrm{O}_{3}$ & Cladosporium sp. JJM22 & & & {$[88]$} \\
\hline $\begin{array}{l}\text { (-)-trans-(3R,4R)-3,4,8-Trihydroxy-6,7- } \\
\text { dimethyl-3,4- dihydronaphthalen-1 }(2 H) \text {-one } \\
\text { (157) }\end{array}$ & 222 & $\mathrm{C}_{12} \mathrm{H}_{14} \mathrm{O}_{4}$ & Cladosporium sp. JJM22 & $\begin{array}{l}\text { Ceriops tagal } \\
\text { (Mangrove plant, Rhizophoraceae) }\end{array}$ & $\begin{array}{l}\text { South China Sea, Dongzhaigang, Hainan, } \\
\text { China }\end{array}$ & {$[88]$} \\
\hline $\begin{array}{l}(3 R, 4 R)-3,4 \text {-Dihydro-3,4,8-trihydroxy-1(2H)- } \\
\text { napthalenone } \\
\text { (159) }\end{array}$ & 194 & $\mathrm{C}_{10} \mathrm{H}_{10} \mathrm{O}_{4}$ & Cladosporium sp. JJM22 & $\begin{array}{l}\text { Ceriops tagal } \\
\text { (Mangrove plant, Rhizophoraceae) }\end{array}$ & $\begin{array}{l}\text { South China Sea, Dongzhaigang, Hainan, } \\
\text { China }\end{array}$ & [88] \\
\hline Aladothalen (160) & 194 & $\mathrm{C}_{10} \mathrm{H}_{10} \mathrm{O}_{4}$ & $\begin{array}{l}\text { Cladosporium sp. HDN17-58 } \\
\text { Cladosporium sp. HDN17-58 }\end{array}$ & $\begin{array}{l}\text { Deep-sea sediment } \\
\text { Deep-sea sediment }\end{array}$ & $\begin{array}{l}\text { Western Pacific Ocean, China } \\
\text { Western Pacific Ocean, China }\end{array}$ & $\begin{array}{l}{[89]} \\
{[89]}\end{array}$ \\
\hline \multicolumn{7}{|l|}{ 8. Perylenequinones } \\
\hline Altertoxin VIII (161) & 304 & $\mathrm{C}_{20} \mathrm{H}_{16} \mathrm{O}_{3}$ & Cladosporium sp. KFD33 & \multirow{3}{*}{$\begin{array}{l}\text { Blood cockle } \\
\text { (Bivalve mollusk, Cardiidae) } \\
\text { Blood cockle } \\
\text { (Bivalve mollusk, Cardiidae) } \\
\text { Blood cockle } \\
\text { (Bivalve mollusk, Cardiidae) } \\
\text { Blood cockle } \\
\text { (Bivalve mollusk, Cardiidae) }\end{array}$} & Haikou Bay, Hainan, China & [85] \\
\hline Altertoxin IX (162) & 290 & $\mathrm{C}_{20} \mathrm{H}_{18} \mathrm{O}_{2}$ & Cladosporium sp. KFD33 & & Haikou Bay, China & [85] \\
\hline Altertoxin XI (164) & 304 & $\mathrm{C}_{21} \mathrm{H}_{20} \mathrm{O}_{2}$ & Cladosporium sp. KFD33 & & Haikou Bay, China & [85] \\
\hline \multicolumn{7}{|l|}{ 9. Naphthalene derivatives } \\
\hline 8-Methoxynaphthalen-1-ol (165) & 174 & $\mathrm{C}_{11} \mathrm{H}_{10} \mathrm{O}_{2}$ & Cladosporium sp. JJM22 & \multirow{8}{*}{$\begin{array}{l}\text { Ceriops tagal } \\
\text { (Mangrove plant, Rhizophoraceae) } \\
\text { Ceriops tagal } \\
\text { (Mangrove plant, Rhizophoraceae) } \\
\text { Ceriops tagal } \\
\text { (Mangrove plant, Rhizophoraceae) } \\
\text { Ceriops tagal } \\
\text { (Mangrove plant, Rhizophoraceae) } \\
\text { Ceriops tagal } \\
\text { (Mangrove plant, Rhizophoraceae) } \\
\text { Ceriops tagal } \\
\text { (Mangrove plant, Rhizophoraceae) } \\
\text { Ceriops tagal } \\
\text { (Mangrove plant, Rhizophoraceae) } \\
\text { Ceriops tagal } \\
\text { (Mangrove plant, Rhizophoraceae) }\end{array}$} & South China Sea, China & {$[90]$} \\
\hline \multirow[t]{3}{*}{ 1,8-Dimethoxynaphthalene (166) } & 188 & $\mathrm{C}_{12} \mathrm{H}_{12} \mathrm{O}_{2}$ & Cladosporium sp. JJM22 & & $\begin{array}{l}\text { South China Sea, Dongzhaigang, Hainan, } \\
\text { China }\end{array}$ & {$[88]$} \\
\hline & & & Cladosporium sp. JJM22 & & South China Sea, China & {$[90]$} \\
\hline & & & Cladosporium sp. JJM22 & & South China Sea, China & {$[91]$} \\
\hline 4-Methoxynaphthalene-1,5-diol (167) & 190 & $\mathrm{C}_{11} \mathrm{H}_{10} \mathrm{O}_{3}$ & Cladosporium sp. JJM22 & & South China Sea, China & {$[91]$} \\
\hline 8-Methoxynaphthalene-1,7-diol (168) & 190 & $\mathrm{C}_{11} \mathrm{H}_{10} \mathrm{O}_{3}$ & Cladosporium sp. JJM22 & & South China Sea, China & {$[91]$} \\
\hline Cladonaphchrom A (169) & 350 & $\mathrm{C}_{22} \mathrm{H}_{22} \mathrm{O}_{4}$ & Cladosporium sp. JJM22 & & South China Sea, China & {$[90]$} \\
\hline Cladonaphchrom B (170) & 350 & $\mathrm{C}_{22} \mathrm{H}_{22} \mathrm{O}_{4}$ & Cladosporium sp. JJM22 & & South China Sea, China & {$[90]$} \\
\hline
\end{tabular}


Table 1. Cont

\begin{tabular}{|c|c|c|c|c|c|c|}
\hline Compound Name & Mol. Wt. & Mol. Formula & Fungal Source & Host (Sample, Family) & Place & Ref \\
\hline \multicolumn{7}{|l|}{ 10. Xanthones } \\
\hline $\begin{array}{l}\text { 8-Hydroxy-6-methylxanthone-1-carboxylic } \\
\text { acid (171) }\end{array}$ & 270 & $\mathrm{C}_{15} \mathrm{H}_{10} \mathrm{O}_{5}$ & C. halotolerans GXIMD 02502 & $\begin{array}{l}\text { Porites lutea } \\
\text { (Stony coral, Poritidae) }\end{array}$ & $\begin{array}{l}\text { Weizhou Islands coral reef, Guangxi Zhuang } \\
\text { autonomous region, China }\end{array}$ & [92] \\
\hline $\begin{array}{l}\text { Methyl 8-hydroxy-6-methyl-9- } \\
\text { oxo-9H-xanthene-1-carboxylate (172) }\end{array}$ & 284 & $\mathrm{C}_{16} \mathrm{H}_{12} \mathrm{O}_{5}$ & C. halotolerans GXIMD 02502 & $\begin{array}{l}\text { Porites lutea } \\
\text { (Stony coral, Poritidae) }\end{array}$ & $\begin{array}{l}\text { Weizhou Islands coral reef, Guangxi Zhuang } \\
\text { autonomous region, China }\end{array}$ & [92] \\
\hline $\begin{array}{l}\text { Methyl 8-hydroxy-6- (hydroxymethyl)-9-oxo- } \\
\text { 9H-xanthene-1-carboxylate } \\
\text { (173) }\end{array}$ & 300 & $\mathrm{C}_{16} \mathrm{H}_{12} \mathrm{O}_{6}$ & C. halotolerans GXIMD 02502 & $\begin{array}{l}\text { Porites lutea } \\
\text { (Stony coral, Poritidae) }\end{array}$ & $\begin{array}{l}\text { Weizhou Islands coral reef, Guangxi Zhuang } \\
\text { autonomous region, China }\end{array}$ & [92] \\
\hline Vertixanthone (174) & 270 & $\mathrm{C}_{15} \mathrm{H}_{10} \mathrm{O}_{5}$ & C. halotolerans GXIMD 02502 & $\begin{array}{l}\text { Porites lutea } \\
\text { (Stony coral, Poritidae) }\end{array}$ & $\begin{array}{l}\text { Weizhou Islands coral reef, Guangxi Zhuang } \\
\text { autonomous region, China }\end{array}$ & [92] \\
\hline $\begin{array}{l}\text { 3,8-Dihydroxy-6-methyl-9-oxo-9H- } \\
\text { xanthene-1-Carboxylate } \\
\text { (176) }\end{array}$ & 300 & $\mathrm{C}_{16} \mathrm{H}_{12} \mathrm{O}_{6}$ & C. halotolerans GXIMD 02502 & $\begin{array}{l}\text { Porites lutea } \\
\text { (Stony coral, Poritidae) }\end{array}$ & $\begin{array}{l}\text { Weizhou Islands coral reef, Guangxi Zhuang } \\
\text { autonomous region, China }\end{array}$ & [92] \\
\hline Conioxanthone A (177) & 316 & $\mathrm{C}_{16} \mathrm{H}_{12} \mathrm{O}_{7}$ & C. halotolerans GXIMD 02502 & $\begin{array}{l}\text { Porites lutea } \\
\text { (Stony coral, Poritidae) }\end{array}$ & $\begin{array}{l}\text { Weizhou Islands coral reef, Guangxi Zhuang } \\
\text { autonomous region, China }\end{array}$ & [92] \\
\hline \multicolumn{7}{|l|}{ 11. Tropolones } \\
\hline Malettinin A (178) & 288 & $\mathrm{C}_{16} \mathrm{H}_{16} \mathrm{O}_{5}$ & Cladosporium sp. KF501 & Water sample & German Wadden Sea & [93] \\
\hline Malettinin B (179) & 292 & $\mathrm{C}_{16} \mathrm{H}_{20} \mathrm{O}_{5}$ & Cladosporium sp. KF501 & Water sample & German Wadden Sea & [93] \\
\hline \multicolumn{7}{|l|}{ 12. Binaphthopyrones } \\
\hline Cladosporinone (182) & 650 & $\mathrm{C}_{33} \mathrm{H}_{30} \mathrm{O}_{14}$ & C. cladosporioides & \multirow{4}{*}{$\begin{array}{l}\text { Sediment of a hypersaline lake El } \\
\text { Hamra } \\
\text { Sediment of a hypersaline lake El } \\
\text { Hamra } \\
\text { Sediment of a hypersaline lake El } \\
\text { Hamra } \\
\text { Sediment of a hypersaline lake El } \\
\text { Hamra }\end{array}$} & Wadi el Natrun, Egypt & [94] \\
\hline Viriditoxin (183) & 662 & $\mathrm{C}_{34} \mathrm{H}_{30} \mathrm{O}_{14}$ & C. cladosporioides & & Wadi el Natrun, Egypt & [94] \\
\hline Viriditoxin derivative 1 (184) & 646 & $\mathrm{C}_{34} \mathrm{H}_{30} \mathrm{O}_{13}$ & C. cladosporioides & & Wadi el Natrun, Egypt & [94] \\
\hline Viriditoxin derivative 2 (185) & 646 & $\mathrm{C}_{34} \mathrm{H}_{30} \mathrm{O}_{13}$ & C. cladosporioides & & Wadi el Natrun, Egypt & [94] \\
\hline \multicolumn{7}{|l|}{ 13. Benzopyranes, benzopyrones, and pyrones } \\
\hline $\begin{array}{l}\text { (2S)-5-Hydroxy-2-methyl-chroman-4-one } \\
\text { (186) }\end{array}$ & 178 & $\mathrm{C}_{10} \mathrm{H}_{10} \mathrm{O}_{3}$ & Cladosporium sp. JJM22 & \multirow{3}{*}{$\begin{array}{l}\text { Ceriops tagal } \\
\text { (Mangrove plant, Rhizophoraceae) } \\
\text { Ceriops tagal } \\
\text { (Mangrove plant, Rhizophoraceae) } \\
\text { Excoecaria agallocha } \\
\text { (Mangrove plant, Euphorbiaceae) }\end{array}$} & $\begin{array}{l}\text { South China Sea, Dongzhaigang, Hainan, } \\
\text { China }\end{array}$ & [88] \\
\hline (R)-5-Hydroxy-2-methylchroman-4-one (187) & 178 & $\mathrm{C}_{10} \mathrm{H}_{10} \mathrm{O}_{3}$ & Cladosporium sp. JJM22 & & South China Sea, China & [90] \\
\hline \multirow{3}{*}{$\begin{array}{l}\text { (2R)-7-O- } \alpha \text {-D-Ribofuranosyl-5-hydroxy-2- } \\
\text { methyl chroman-4-one } \\
\text { (188) }\end{array}$} & \multirow{3}{*}{326} & \multirow{3}{*}{$\mathrm{C}_{15} \mathrm{H}_{18} \mathrm{O}_{8}$} & Cladosporium sp. OUCMDZ-302 & & Wenchang, Hainan, China & [95] \\
\hline & & & Cladosporium sp. OUCMDZ-302 & $\begin{array}{l}\text { Excoecaria agallocha } \\
\text { (Mangrove plant, Euphorbiaceae) }\end{array}$ & Wenchang, Hainan, China & [95] \\
\hline & & & Cladosporium sp. JJM22 & $\begin{array}{l}\text { Ceriops tagal } \\
\text { (Mangrove plant, Rhizophoraceae) }\end{array}$ & South China Sea, China & [91] \\
\hline
\end{tabular}


Table 1. Cont.

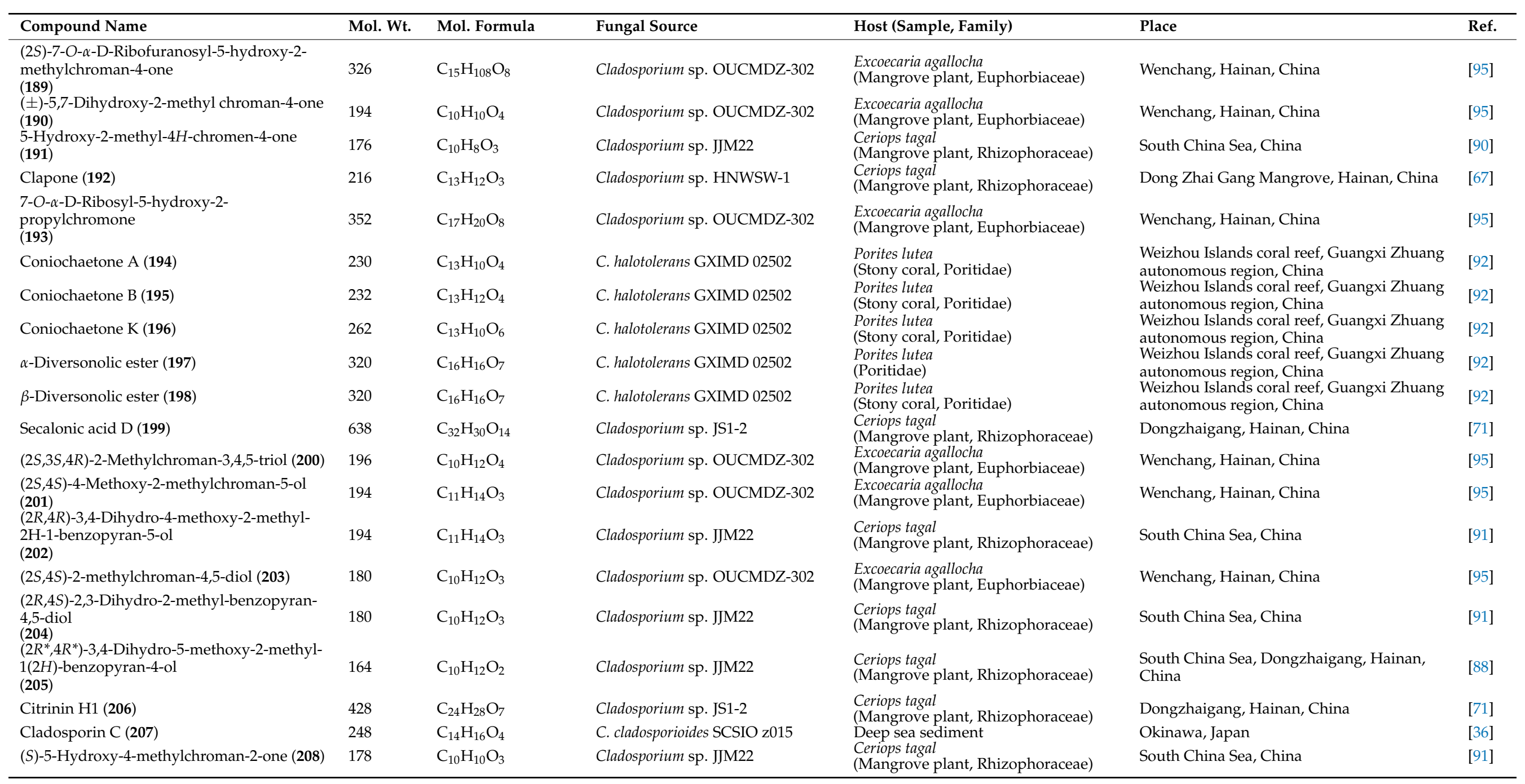


Table 1. Cont.

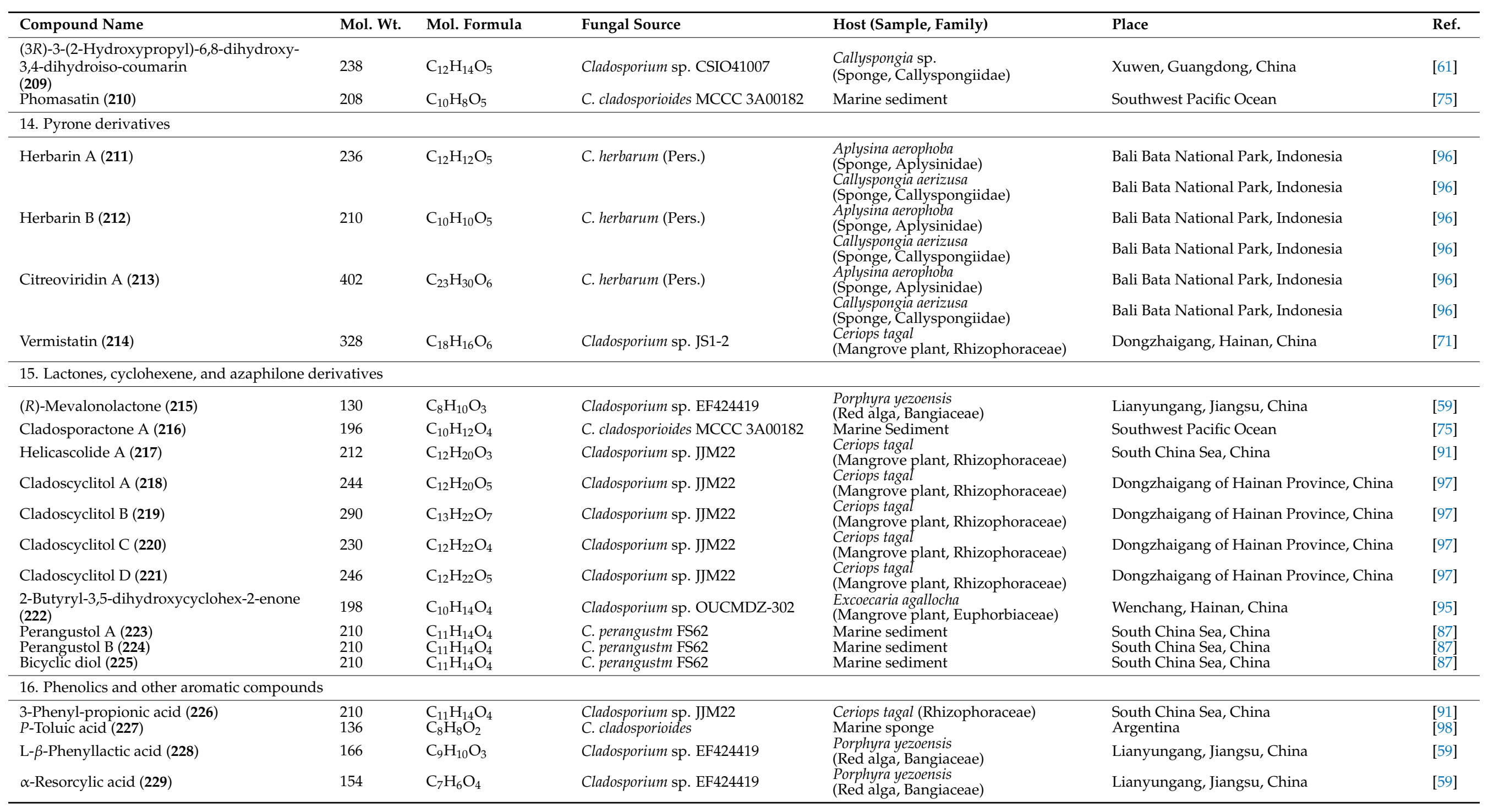


Table 1. Cont.

\begin{tabular}{|c|c|c|c|c|c|c|}
\hline Compound Name & Mol. Wt. & Mol. Formula & Fungal Source & Host (Sample, Family) & Place & Ref. \\
\hline Phenylacetic acid (230) & 136 & $\mathrm{C}_{8} \mathrm{H}_{8} \mathrm{O}_{2}$ & Cladosporium sp. EF424419 & $\begin{array}{l}\text { Porphyra yezoensis } \\
\text { (Red alga, Bangiaceae) }\end{array}$ & Lianyungang, Jiangsu, China & [59] \\
\hline P-Hydroxyphenylacetic acid (231) & 152 & $\mathrm{C}_{8} \mathrm{H}_{8} \mathrm{O}_{3}$ & Cladosporium sp. EF424419 & $\begin{array}{l}\text { Porphyra yezoensis } \\
\text { (Red alga, Bangiaceae) }\end{array}$ & Lianyungang, Jiangsu, China & [59] \\
\hline $\begin{array}{l}\text { Cinnamic acid (3-Phenyl-2-propenoic acid) } \\
\text { (232) }\end{array}$ & 148 & $\mathrm{C}_{9} \mathrm{H}_{8} \mathrm{O}_{2}$ & Cladosporium sp. F14 & Seawater from the mangrove stand & Kei Ling Ha Lo Wai, Sai Kung, China & {$[60]$} \\
\hline $\begin{array}{l}\text { 3-(2,3-Dihydroxy phenoxy) butanoic acid } \\
\text { (233) }\end{array}$ & 212 & $\mathrm{C}_{10} \mathrm{H}_{12} \mathrm{O}_{5}$ & Cladosporium sp. OUCMDZ-302 & $\begin{array}{l}\text { Excoecaria agallocha } \\
\text { (Mangrove plant, Euphorbiaceae) }\end{array}$ & Wenchang, Hainan, China & [95] \\
\hline$P$-Hydroxy benzoic acid methyl ester (234) & 152 & $\mathrm{C}_{8} \mathrm{H}_{8} \mathrm{O}_{3}$ & Cladosporium sp. EF424419 & $\begin{array}{l}\text { Porphyra yezoensis } \\
\text { (Red alga, Bangiaceae) }\end{array}$ & Lianyungang, Jiangsu, China & [59] \\
\hline $\begin{array}{l}\text { Methyl (3S)-3-(2,3-dihydroxy } \\
\text { phenyloxy)butanoate (235) }\end{array}$ & 226 & $\mathrm{C}_{11} \mathrm{H}_{14} \mathrm{O}_{5}$ & Cladosporium sp. OUCMDZ-302 & $\begin{array}{l}\text { Excoecaria agallocha } \\
\text { (Mangrove plant, Euphorbiaceae) }\end{array}$ & Wenchang, Hainan, China & [95] \\
\hline$P$-Hydroxyphenylethyl alcohol (236) & 138 & $\mathrm{C}_{8} \mathrm{H}_{10} \mathrm{O}_{2}$ & Cladosporium sp. EF424419 & $\begin{array}{l}\text { Porphyra yezoensis } \\
\text { (Red alga, Bangiaceae) }\end{array}$ & Lianyungang, Jiangsu Province, China & [59] \\
\hline$P$-Hydroxybenzyl alcohol (237) & 142 & $\mathrm{C}_{7} \mathrm{H}_{8} \mathrm{O}_{2}$ & Cladosporium sp. EF424419 & $\begin{array}{l}\text { Porphyra yezoensis } \\
\text { (Red alga, Bangiaceae) }\end{array}$ & Lianyungang, Jiangsu Province, China & [59] \\
\hline 2-Phenylethanol (238) & 122 & $\mathrm{C}_{8} \mathrm{H}_{10} \mathrm{O}$ & Cladosporium sp. F14 & Seawater from the mangrove stand & Kei Ling Ha Lo Wai, Sai Kung, China & [60] \\
\hline $\begin{array}{l}\text { 4-O- } \alpha-D-\text { Ribofuranose-3-hydroxymethyl-2- } \\
\text { pentyl- phenol } \\
\text { (239) }\end{array}$ & 342 & $\mathrm{C}_{17} \mathrm{H}_{26} \mathrm{O}_{7}$ & Cladosporium sp. JJM22 & $\begin{array}{l}\text { Ceriops tagal } \\
\text { (Mangrove plant, Rhizophoraceae) }\end{array}$ & $\begin{array}{l}\text { South China Sea, Dongzhaigang, Hainan, } \\
\text { China }\end{array}$ & [88] \\
\hline $\begin{array}{l}\text { 4-O- } \alpha \text {-D-Ribofuranose-2-pentyl-3- } \\
\text { phemethylol } \\
\text { (240) }\end{array}$ & 326 & $\mathrm{C}_{17} \mathrm{H}_{26} \mathrm{O}_{6}$ & Cladosporium sp. JJM22 & $\begin{array}{l}\text { Ceriops tagal } \\
\text { (Mangrove plant, Rhizophoraceae) }\end{array}$ & Dongzhaigang of Hainan Province, China & [97] \\
\hline Clavatol (241) & 180 & $\mathrm{C}_{10} \mathrm{H}_{12} \mathrm{O}_{3}$ & Cladosporium sp.MFC353-b & $\begin{array}{l}\text { Chondria crassicualis } \\
\text { (Red alga, Rhodomelaceae) }\end{array}$ & Yokji Island, Kyeongnam, Korea & [65] \\
\hline $\begin{array}{l}\text { 1-(3,5-Dihydroxy-4-methylphenyl)propan-2- } \\
\text { one } \\
\text { (242) }\end{array}$ & 180 & $\mathrm{C}_{10} \mathrm{H}_{12} \mathrm{O}_{3}$ & C. perangustm FS62 & Marine sediment & South China Sea, china & [87] \\
\hline$\alpha$-Acetylorcinol (243) & 166 & $\mathrm{C}_{9} \mathrm{H}_{10} \mathrm{O}_{3}$ & C. perangustm FS62 & Marine sediment & South China Sea, china & [87] \\
\hline 1-(2,6-Dihydroxyphenyl) ethanone (244) & 152 & $\mathrm{C}_{8} \mathrm{H}_{8} \mathrm{O}_{3}$ & Cladosporium sp. OUCMDZ-302 & $\begin{array}{l}\text { Excoecaria agallocha } \\
\text { (Mangrove plant, Euphorbiaceae) }\end{array}$ & Wenchang, Hainan, China & [95] \\
\hline 1-(2,6-Hihydroxyphenyl)-1-butanone (245) & 180 & $\mathrm{C}_{10} \mathrm{H}_{12} \mathrm{O}_{3}$ & Cladosporium sp. OUCMDZ-302 & $\begin{array}{l}\text { Excoecaria agallocha } \\
\text { (Mangrove plant, Euphorbiaceae) }\end{array}$ & Wenchang, Hainan, China & [95] \\
\hline $\begin{array}{l}\text { (R)-3-Methoxyl-1-(2,6-dihydroxyphenyl)- } \\
\text { butan-1-one } \\
\text { (246) }\end{array}$ & 210 & $\mathrm{C}_{11} \mathrm{H}_{14} \mathrm{O}_{4}$ & Cladosporium sp. JJM22 & Ceriops tagal (Rhizophoraceae) & South China Sea, China & [91] \\
\hline $\begin{array}{l}\text { Cladosporin D (247) } \\
\text { (2S)-7, } 4^{\prime} \text {-dihydroxy-5-methoxy-8- }(\gamma, \gamma-\end{array}$ & 224 & $\mathrm{C}_{12} \mathrm{H}_{16} \mathrm{O}_{4}$ & C. cladosporioides SCSIO z015 & Deep sea sediment & Okinawa, Japan & [36] \\
\hline dimethylallyl)-flavanone & 354 & $\mathrm{C}_{21} \mathrm{H}_{22} \mathrm{O}_{5}$ & Cladosporium sp. TPU1507 & Unidentified marine sponge & Manado, Indonesia & [68] \\
\hline Bis(2-Ethylhexyl)phthalate (249) & 390 & $\mathrm{C}_{24} \mathrm{H}_{38} \mathrm{O}_{4}$ & Cladosporium sp. F14 & Seawater from the mangrove stand & Kei Ling Ha Lo Wai, Sai Kung, China & [60] \\
\hline Herbaric acid (250) & 196 & $\mathrm{C}_{9} \mathrm{H}_{8} \mathrm{O}_{5}$ & C. herbarum (Pers.) & $\begin{array}{l}\text { (allyspongia aerizusa } \\
\text { (Sponge, Callyspongiidae) }\end{array}$ & Bali Bata National Park, Indonesia & [96] \\
\hline Cladosacid (251) & 250 & $\mathrm{C}_{15} \mathrm{H}_{22} \mathrm{O}_{3}$ & Cladosporium sp. OUCMDZ-1635 & Unidentified sponge & Xisha Islands, China & [56] \\
\hline 1,1'-Dioxine-2,2'-dipropionic acid (252) & 228 & $\mathrm{C}_{10} \mathrm{H}_{12} \mathrm{O}_{6}$ & Cladosporium sp. JS1-2 & $\begin{array}{l}\text { Ceriops tagal } \\
\text { (Mangrove, plant, Rhizophoraceae) }\end{array}$ & Dongzhaigang, Hainan, China & [71] \\
\hline Sumiki's acid (253) & 142 & $\mathrm{C}_{6} \mathrm{H}_{6} \mathrm{O}_{4}$ & C. herbarum (Pers.) & $\begin{array}{l}\text { Callyspongia aerizusa } \\
\text { (Sponge, Callyspongiidae) }\end{array}$ & Bali Bata National Park, Indonesia & [73] \\
\hline
\end{tabular}


Table 1. Cont.

\begin{tabular}{|c|c|c|c|c|c|c|}
\hline Compound Name & Mol. Wt. & Mol. Formula & Fungal Source & Host (Sample, Family) & Place & Ref. \\
\hline Acetyl Sumiki's acid (254) & 184 & $\mathrm{C}_{8} \mathrm{H}_{8} \mathrm{O}_{5}$ & C. herbarum (Pers.) & $\begin{array}{l}\text { Callyspongia aerizusa } \\
\text { (Sponge, Callyspongiidae) }\end{array}$ & Bali Bata National Park, Indonesia & {$[74]$} \\
\hline \multicolumn{7}{|l|}{ 17. Sterols and terpenes } \\
\hline $\begin{array}{l}5 \alpha, 8 \alpha \text {-Epidioxy-24(R)-methyl-cholesta-6,22- } \\
\text { diene-3- } \beta \text {-ol } \\
(\mathbf{2 5 5})\end{array}$ & 428 & $\mathrm{C}_{28} \mathrm{H}_{44} \mathrm{O}_{3}$ & C. sphaerospermum Penz & $\begin{array}{l}\text { Ceramium condi } \\
\text { (Red alga, Ceramiaceae) }\end{array}$ & Ussuriysk Bay, Japan & [99] \\
\hline $\begin{array}{l}5 \alpha, 8 \alpha \text {-Epidioxy-ergosta-6,22E-dien-3 } 3 \text {-ol } \\
(\mathbf{2 5 6 )}\end{array}$ & 428 & $\mathrm{C}_{28} \mathrm{H}_{44} \mathrm{O}_{3}$ & $\begin{array}{l}\text { C.cladosporioides MCCC 3A00182 } \\
\text { Cladosporium sp. WZ-2008-0042 } \\
\text { C. cladosporioides MCCC 3A00182 }\end{array}$ & $\begin{array}{l}\text { Marine sediment } \\
\text { Dichotella gemmacea } \\
\text { (Gorgonian, Ellisellidae) } \\
\text { Marine Sediment }\end{array}$ & $\begin{array}{l}\text { Southwest Pacific Ocean } \\
\text { Weizhou Island coral reef, South China Sea } \\
\text { Southwest Pacific Ocean }\end{array}$ & $\begin{array}{l}{[75]} \\
{[100]} \\
{[75]}\end{array}$ \\
\hline $\begin{array}{l}5 \alpha, 8 \alpha \text {-Epidioxy-ergosta- } 6,9,22 E \text {-triene- } 3 \beta \text {-ol } \\
(\mathbf{2 5 8})\end{array}$ & 426 & $\mathrm{C}_{28} \mathrm{H}_{42} \mathrm{O}_{3}$ & Cladosporium sp. WZ-2008-0042 & $\begin{array}{l}\text { Dichotella gemmacea } \\
\text { (Gorgonian, Ellisellidae) }\end{array}$ & Weizhou Island coral reef, South China Sea & [100] \\
\hline $\begin{array}{l}3 \beta, 5 \alpha, 6 \beta \text { - } \\
\text { Trihydroxyergosta- } 7,22 \text {-diene }=\text { Cerevisterol } \\
\text { (259) }\end{array}$ & 430 & $\mathrm{C}_{28} \mathrm{H}_{46} \mathrm{O}_{3}$ & Cladosporium sp. SCSIO41007 & $\begin{array}{l}\text { Callyspongia sp. } \\
\text { (Sponge, Callyspongiidae) }\end{array}$ & Xuwen, Guangdong, China & {$[61]$} \\
\hline Ergosta-7,22E-diene- $3 \beta, 5 \alpha, 6 \beta$-triol (260) & 430 & $\mathrm{C}_{28} \mathrm{H}_{46} \mathrm{O}_{3}$ & Cladosporium sp. WZ-2008-0042 & $\begin{array}{l}\text { Dichotella gemmacea } \\
\text { (Gorgonian, Ellisellidae) }\end{array}$ & Weizhou Island coral reef, South China Sea & {$[100]$} \\
\hline 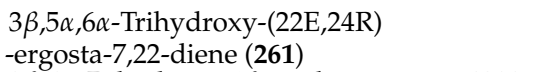 & 430 & $\mathrm{C}_{28} \mathrm{H}_{46} \mathrm{O}_{3}$ & C. cladosporioides MCCC 3A00182 & Marine Sediment & Southwest Pacific Ocean & {$[75]$} \\
\hline $\begin{array}{l}3 \beta, 5 \alpha \text {-Dihydroxy- } 6 \beta \text {-methoxyergosta-7,22- } \\
\text { diene } \\
\text { (262) }\end{array}$ & 444 & $\mathrm{C}_{29} \mathrm{H}_{48} \mathrm{O}_{3}$ & Cladosporium sp. WZ-2008-0042 & $\begin{array}{l}\text { Dichotella gemmacea } \\
\text { (Gorgonian, Ellisellidae) }\end{array}$ & Weizhou Island coral reef, South China Sea & [100] \\
\hline Ergosterol (263) & 396 & $\mathrm{C}_{28} \mathrm{H}_{44} \mathrm{O}$ & Cladosporium sp. WZ-2008-0042 & $\begin{array}{l}\text { Dichotella gemmacea } \\
\text { (Gorgonian, Ellisellidae) }\end{array}$ & Weizhou Island coral reef, South China Sea & [100] \\
\hline Cladosporisteroid A (264) & 460 & $\mathrm{C}_{28} \mathrm{H}_{44} \mathrm{O}_{5}$ & Cladosporium sp. SCSIO41007 & $\begin{array}{l}\text { Callyspongia sp. } \\
\text { (Sponge, Callyspongiidae) }\end{array}$ & Xuwen, Guangdong, China & {$[61]$} \\
\hline \multirow[t]{2}{*}{$\begin{array}{l}3 \beta, 5 \alpha, 9 \alpha \text {-Trihydroxy-( } 22 E, 24 R) \text {-ergosta-7,22- } \\
\text { diene-6-one } \\
(\mathbf{2 6 5})\end{array}$} & 444 & $\mathrm{C}_{28} \mathrm{H}_{44} \mathrm{O}_{4}$ & Cladosporium sp. SCSIO41007 & $\begin{array}{l}\text { Callyspongia sp. } \\
\text { (Sponge, Callyspongiidae) }\end{array}$ & Xuwen, Guangdong, China & {$[61]$} \\
\hline & & & C. cladosporioides MCCC 3A00182 & Marine Sediment & Southwest Pacific Ocean & {$[75]$} \\
\hline $\begin{array}{l}\text { 3 } \beta, 5 \alpha \text {-Dihydroxy-(22E,24R)-ergosta-7,22- } \\
\text { diene-6-one } \\
\text { (266) }\end{array}$ & 428 & $\mathrm{C}_{28} \mathrm{H}_{44} \mathrm{O}_{3}$ & C. cladosporioides MCCC 3A00182 & Marine Sediment & Southwest Pacific Ocean & {$[75]$} \\
\hline Stigma-5-en-3-O- $\beta$-glucopyranoside (267) & 576 & $\mathrm{C}_{35} \mathrm{H}_{60} \mathrm{O}_{6}$ & Cladosporium sp. WZ-2008-0042 & $\begin{array}{l}\text { Dichotella gemmacea } \\
\text { (Gorgonian, Ellisellidae) }\end{array}$ & Weizhou Island coral reef, South China Sea & [100] \\
\hline \multirow[t]{4}{*}{$\begin{array}{l}3 \alpha \text {-Hydroxy-pregna-7-ene-6,20-dione }= \\
\text { Cladosporisteroid B }(\mathbf{2 6 8})\end{array}$} & 330 & $\mathrm{C}_{21} \mathrm{H}_{30} \mathrm{O}_{3}$ & Cladosporium sp. WZ-2008-0042 & $\begin{array}{l}\text { Dichotella gemmacea } \\
\text { (Gorgonian, Ellisellidae) }\end{array}$ & Weizhou Island coral reef, South China Sea & [100] \\
\hline & & & Cladosporium sp. SCSIO41007 & $\begin{array}{l}\text { Callyspongia sp. } \\
\text { (Sponoe Callysponoiidae) }\end{array}$ & Xuwen, Guangdong, China & {$[61]$} \\
\hline & & & C. cladosporioides MCCC 3A00182 & Marine Sediment & Southwest Pacific Ocean & [75] \\
\hline & & & C. sphaerospermum SW67 & $\begin{array}{l}\text { Hydractinia echinata } \\
\text { (Hydroid, Hydractiniidae) }\end{array}$ & South Korea & [101] \\
\hline
\end{tabular}


Table 1. Cont.

\begin{tabular}{|c|c|c|c|c|c|c|}
\hline Compound Name & Mol. Wt. & Mol. Formula & Fungal Source & Host (Sample, Family) & Place & Ref. \\
\hline Cladosporisteroid C (269) & 374 & $\mathrm{C}_{23} \mathrm{H}_{34} \mathrm{O}_{4}$ & Cladosporium sp. SCSIO41007 & \multirow{2}{*}{$\begin{array}{l}\text { Callyspongia sp. } \\
\text { (Sponge, Callyspongiidae) } \\
\text { Callyspongia sp. } \\
\text { (Sponge, Callyspongiidae) }\end{array}$} & Xuwen, Guangdong, China & [61] \\
\hline Pregn-7-dien-3,6,20-trione (270) & 328 & $\mathrm{C}_{21} \mathrm{H}_{28} \mathrm{O}_{3}$ & Cladosporium sp. SCSIO41007 & & Xuwen, Guangdong, China & [61] \\
\hline \multicolumn{4}{|l|}{ 18. Alcohols and aldehydes } & \multicolumn{3}{|l|}{$70.43 \mu \mathrm{g} / \mathrm{mL}\left(\mathrm{EC}_{50}\right)$} \\
\hline Compound (271) & 434 & $\mathrm{C}_{30} \mathrm{H}_{58} \mathrm{O}$ & Cladosporium sp. & Marine sediment & San Antonio Oeste, Río Negro, Argentina & [102] \\
\hline Compound (272) & 458 & $\mathrm{C}_{32} \mathrm{H}_{58} \mathrm{O}$ & Cladosporium sp. & Marine sediment & San Antonio Oeste, Río Negro, Argentina & [102] \\
\hline Compound (273) & 458 & $\mathrm{C}_{32} \mathrm{H}_{58} \mathrm{O}$ & Cladosporium sp. & Marine sediment & San Antonio Oeste, Río Negro, Argentina & [102] \\
\hline Compound (274) & 458 & $\mathrm{C}_{32} \mathrm{H}_{58} \mathrm{O}$ & Cladosporium sp. & Marine sediment & San Antonio Oeste, Río Negro, Argentina & [102] \\
\hline Compound (275) & 460 & $\mathrm{C}_{32} \mathrm{H}_{60} \mathrm{O}$ & Cladosporium sp. & Marine sediment & San Antonio Oeste, Río Negro, Argentina & [102] \\
\hline Compound (277) & 462 & $\mathrm{C}_{32} \mathrm{H}_{62} \mathrm{O}$ & Cladosporium sp. & Marine sediment & San Antonio Oeste, Río Negro, Argentina & [102] \\
\hline Compound (278) & 462 & $\mathrm{C}_{32} \mathrm{H}_{62} \mathrm{O}$ & Cladosporium sp. & Marine sediment & San Antonio Oeste, Río Negro, Argentina & [102] \\
\hline Compound (279) & 482 & $\mathrm{C}_{34} \mathrm{H}_{58} \mathrm{O}$ & Cladosporium sp. & Marine sediment & San Antonio Oeste, Río Negro, Argentina & [102] \\
\hline Compound (280) & 484 & $\mathrm{C}_{34} \mathrm{H}_{60} \mathrm{O}$ & Cladosporium sp. & Marine sediment & San Antonio Oeste, Río Negro, Argentina & [102] \\
\hline Compound (281) & 484 & $\mathrm{C}_{34} \mathrm{H}_{60} \mathrm{O}$ & Cladosporium sp. & Marine sediment & San Antonio Oeste, Río Negro, Argentina & [102] \\
\hline Compound (282) & 484 & $\mathrm{C}_{34} \mathrm{H}_{60} \mathrm{O}$ & Cladosporium sp. & Marine sediment & San Antonio Oeste, Río Negro, Argentina & [102] \\
\hline Compound (283) & 484 & $\mathrm{C}_{34} \mathrm{H}_{60} \mathrm{O}$ & Cladosporium sp. & Marine sediment & San Antonio Oeste, Río Negro, Argentina & [102] \\
\hline Compound (284) & 486 & $\mathrm{C}_{34} \mathrm{H}_{62} \mathrm{O}$ & Cladosporium sp. & Marine sediment & San Antonio Oeste, Río Negro, Argentina & [102] \\
\hline$(2 S, 3 S, 4 E)$-Hepta-4,6-diene-2,3-diol (285) & 128 & $\mathrm{C}_{7} \mathrm{H}_{12} \mathrm{O}_{2}$ & Cladosporium sp. OUCMDZ-302 & \multirow{2}{*}{$\begin{array}{l}\text { Excoecaria agallocha } \\
\text { (Mangrove plant, Euphorbiaceae) } \\
\text { Excoecaria agallocha } \\
\text { (Mangrove plant, Euphorbiaceae) }\end{array}$} & Wenchang, Hainan, China & [95] \\
\hline (3E,8E,6S)-Undeca-3,8,10-trien-1,6-diol (286) & 182 & $\mathrm{C}_{11} \mathrm{H}_{18} \mathrm{O}_{2}$ & Cladosporium sp. OUCMDZ-302 & & Wenchang, Hainan, China & {$[95]$} \\
\hline
\end{tabular}

Table 2. Biological activity of secondary metabolites isolated from Cladosporium species.

\begin{tabular}{|c|c|c|c|c|c|}
\hline Compound Name & Biological Activity & Assay, Organism, or Cell Line & Biological Results & Positive Control & Ref. \\
\hline Cladosin C (3) & Antiviral & $\begin{array}{l}\text { Neuraminidase inhibition assay/Influenza A } \\
\text { H1N1 virus }\end{array}$ & $276.0 \mu \mathrm{M}\left(\mathrm{IC}_{50}\right)$ & Ribavirin $131.0 \mu \mathrm{M}\left(\mathrm{IC}_{50}\right)$ & [42] \\
\hline \multirow[t]{5}{*}{ Cladosin I (8) } & Cytotoxicity & MTT/K562 & $4.1 \mu \mathrm{M}\left(\mathrm{IC}_{50}\right)$ & Doxorubicin $0.3 \mu \mathrm{M}\left(\mathrm{IC}_{50}\right)$ & [55] \\
\hline & Cytotoxicity & SEB/HCT-116 & $11.0 \mu \mathrm{M}\left(\mathrm{IC}_{50}\right)$ & Doxorubicin $0.2 \mu \mathrm{M}\left(\mathrm{IC}_{50}\right)$ & [55] \\
\hline & Cytotoxicity & SRB/PC-3 & $13.0 \mu \mathrm{M}\left(\mathrm{IC}_{50}\right)$ & Doxorubicin $1.0 \mu \mathrm{M}\left(\mathrm{IC}_{50}\right)$ & [55] \\
\hline & Cytotoxicity & SRB/SH-SY5Y & $12.0 \mu \mathrm{M}\left(\mathrm{IC}_{50}\right)$ & Doxorubicin $0.1 \mu \mathrm{M}\left(\mathrm{IC}_{50}\right)$ & [55] \\
\hline & Cytotoxicity & SRB/MGC-803 & $19.0 \mu \mathrm{M}\left(\mathrm{IC}_{50}\right)$ & Doxorubicin $0.2 \mu \mathrm{M}\left(\mathrm{IC}_{50}\right)$ & [55] \\
\hline
\end{tabular}


Table 2. Cont

\begin{tabular}{|c|c|c|c|c|c|}
\hline Compound Name & Biological Activity & Assay, Organism, or Cell Line & Biological Results & Positive Control & Ref. \\
\hline \multirow[t]{4}{*}{ Cladosin K (10) } & Cytotoxicity & MTT/K562 & $5.9 \mu \mathrm{M}\left(\mathrm{IC}_{50}\right)$ & Doxorubicin $0.3 \mu \mathrm{M}\left(\mathrm{IC}_{50}\right)$ & {$[55]$} \\
\hline & Cytotoxicity & MTT/HL-60 & $7.5 \mu \mathrm{M}\left(\mathrm{IC}_{50}\right)$ & Doxorubicin $0.2 \mu \mathrm{M}\left(\mathrm{IC}_{50}\right)$ & {$[55]$} \\
\hline & Cytotoxicity & SEB/HCT-116 & $14.0 \mu \mathrm{M}\left(\mathrm{IC}_{50}\right)$ & Doxorubicin $0.2 \mu \mathrm{M}\left(\mathrm{IC}_{50}\right)$ & {$[55]$} \\
\hline & Cytotoxicity & SRB/PC-3 & $18.0 \mu \mathrm{M}\left(\mathrm{IC}_{50}\right)$ & Doxorubicin $1.0 \mu \mathrm{M}\left(\mathrm{IC}_{50}\right)$ & {$[55]$} \\
\hline \multirow[t]{3}{*}{ Cladosporicin A (12) } & Cytotoxicity & SRB/Bt549 & $70.88 \mu \mathrm{M}\left(\mathrm{IC}_{50}\right)$ & Etoposide $1.82 \mu \mathrm{M}\left(\mathrm{IC}_{50}\right)$ & {$[38]$} \\
\hline & Cytotoxicity & SRB/HCC70 & $74.48 \mu \mathrm{M}\left(\mathrm{IC}_{50}\right)$ & Etoposide $1.76 \mu \mathrm{M}\left(\mathrm{IC}_{50}\right)$ & {$[38]$} \\
\hline & Cytotoxicity & SRB/MDA-MB-231 & $75.54 \mu \mathrm{M}\left(\mathrm{IC}_{50}\right)$ & Etoposide $2.27 \mu \mathrm{M}\left(\mathrm{IC}_{50}\right)$ & {$[38]$} \\
\hline \multirow[t]{9}{*}{ Cladodionen (13) } & Cytotoxicity & MTT/K562 & $4.5 \mu \mathrm{M}\left(\mathrm{IC}_{50}\right)$ & Doxorubicin $0.3 \mu \mathrm{M}\left(\mathrm{IC}_{50}\right)$ & {$[55]$} \\
\hline & Cytotoxicity & MTT/HL-60 & $6.6 \mu \mathrm{M}\left(\mathrm{IC}_{50}\right)$ & Doxorubicin $0.2 \mu \mathrm{M}\left(\mathrm{IC}_{50}\right)$ & {$[55]$} \\
\hline & Cytotoxicity & SRB/HCT-116 & $12.0 \mu \mathrm{M}\left(\mathrm{IC}_{50}\right)$ & Doxorubicin $0.2 \mu \mathrm{M}\left(\mathrm{IC}_{50}\right)$ & {$[55]$} \\
\hline & Cytotoxicity & SRB/PC-3 & $11.0 \mu \mathrm{M}\left(\mathrm{IC}_{50}\right)$ & Doxorubicin $1.0 \mu \mathrm{M}\left(\mathrm{IC}_{50}\right)$ & {$[55]$} \\
\hline & Cytotoxicity & SRB/SH-SY5Y & $15.0 \mu \mathrm{M}\left(\mathrm{IC}_{50}\right)$ & Doxorubicin $0.1 \mu \mathrm{M}\left(\mathrm{IC}_{50}\right)$ & {$[55]$} \\
\hline & Cytotoxicity & SRB/MGC-803 & $22.0 \mu \mathrm{M}\left(\mathrm{IC}_{50}\right)$ & Doxorubicin $0.2 \mu \mathrm{M}\left(\mathrm{IC}_{50}\right)$ & {$[55]$} \\
\hline & Cytotoxicity & MTT/MCF-7 & $18.7 \mu \mathrm{M}\left(\mathrm{IC}_{50}\right)$ & Adriamycin $0.67 \mu \mathrm{M}\left(\mathrm{IC}_{50}\right)$ & {$[56]$} \\
\hline & Cytotoxicity & MTT/HeLa & $19.1 \mu \mathrm{M}\left(\mathrm{IC}_{50}\right)$ & Adriamycin $0.32 \mu \mathrm{M}\left(\mathrm{IC}_{50}\right)$ & {$[56]$} \\
\hline & Cytotoxicity & MTT/HL-60 & $9.0 \mu \mathrm{M}\left(\mathrm{IC}_{50}\right)$ & Adriamycin $0.02 \mu \mathrm{M}\left(\mathrm{IC}_{50}\right)$ & {$[56]$} \\
\hline \multirow[t]{4}{*}{ Cladosporiumin I (29) } & Cytotoxicity & SRB/Bt549 & $76.18 \mu \mathrm{M}\left(\mathrm{IC}_{50}\right)$ & Etoposide $1.82 \mu \mathrm{M}\left(\mathrm{IC}_{50}\right)$ & {$[38]$} \\
\hline & Cytotoxicity & SRB/HCC70 & $85.29 \mu \mathrm{M}\left(\mathrm{IC}_{50}\right)$ & Etoposide $1.76 \mu \mathrm{M}\left(\mathrm{IC}_{50}\right)$ & {$[38]$} \\
\hline & Cytotoxicity & SRB/MDA-MB-231 & $82.37 \mu \mathrm{M}\left(\mathrm{IC}_{50}\right)$ & Etoposide $2.27 \mu \mathrm{M}\left(\mathrm{IC}_{50}\right)$ & {$[38]$} \\
\hline & Cytotoxicity & SRB/MDA-MB-468 & $81.44 \mu \mathrm{M}\left(\mathrm{IC}_{50}\right)$ & Etoposide $2.08 \mu \mathrm{M}\left(\mathrm{IC}_{50}\right)$ & {$[38]$} \\
\hline \multirow[t]{4}{*}{ Cladosporiumin J (30) } & Cytotoxicity & SRB/Bt549 & $78.96 \mu \mathrm{M}\left(\mathrm{IC}_{50}\right)$ & Etoposide $1.82 \mu \mathrm{M}\left(\mathrm{IC}_{50}\right)$ & {$[38]$} \\
\hline & Cytotoxicity & SRB/HCC70 & $76.41 \mu \mathrm{M}\left(\mathrm{IC}_{50}\right)$ & Etoposide $1.76 \mu \mathrm{M}\left(\mathrm{IC}_{50}\right)$ & {$[38]$} \\
\hline & Cytotoxicity & SRB/MDA-MB-231 & $79.27 \mu \mathrm{M}\left(\mathrm{IC}_{50}\right)$ & Etoposide $2.27 \mu \mathrm{M}\left(\mathrm{IC}_{50}\right)$ & {$[38]$} \\
\hline & Cytotoxicity & SRB/MDA-MB-468 & $74.64 \mu \mathrm{M}\left(\mathrm{IC}_{50}\right)$ & Etoposide $2.08 \mu \mathrm{M}\left(\mathrm{IC}_{50}\right)$ & {$[38]$} \\
\hline \multirow{4}{*}{ Cyclo-(Val-Pro) (32) } & Insecticidal & Inhibitinon $50 \% /$ B. amphitrite & $37.82 \mu \mathrm{g} / \mathrm{mL}\left(\mathrm{EC}_{50}\right)$ & FSW with DMSO & {$[60]$} \\
\hline & & Lethality $50 \% /$ B. amphitrite & $>200 \mu \mathrm{g} / \mathrm{mL}\left(\mathrm{LC}_{50}\right)$ & FSW with DMSO & {$[60]$} \\
\hline & & Inhibitinon 50\%/B. neritina & $>200 \mu \mathrm{g} / \mathrm{mL}\left(\mathrm{EC}_{50}\right)$ & FSW with DMSO & {$[60]$} \\
\hline & & Lethality $50 \% / B$. neritina & $>200 \mu \mathrm{g} / \mathrm{mL}\left(\mathrm{LC}_{50}\right)$ & FSW with DMSO & {$[60]$} \\
\hline
\end{tabular}


Table 2. Cont

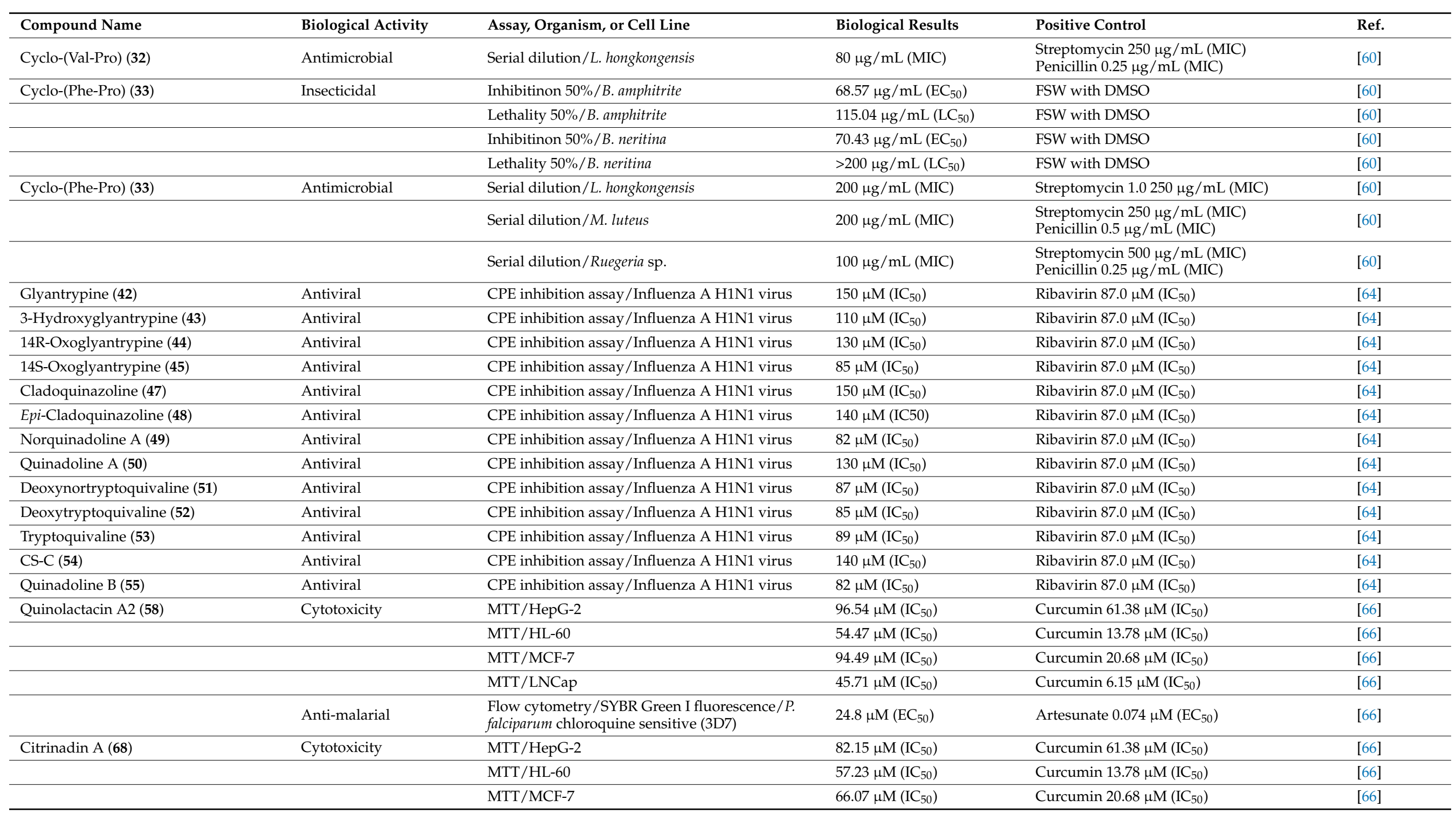


Table 2. Cont.

\begin{tabular}{|c|c|c|c|c|c|}
\hline Compound Name & Biological Activity & Assay, Organism, or Cell Line & Biological Results & Positive Control & Ref. \\
\hline & & MTT/LNCap & $41.42 \mu \mathrm{M}\left(\mathrm{IC}_{50}\right)$ & Curcumin $6.15 \mu \mathrm{M}\left(\mathrm{IC}_{50}\right)$ & [66] \\
\hline & Anti-malarial & $\begin{array}{l}\text { Flow cytometry/SYBR Green I } \\
\text { fluorescence/P. falciparum chloroquine sensitive } \\
\text { (3D7) }\end{array}$ & $>25.0 \mu \mathrm{M}\left(\mathrm{EC}_{50}\right)$ & Artesunate $0.074 \mu \mathrm{M}\left(\mathrm{EC}_{50}\right)$ & [66] \\
\hline \multirow[t]{5}{*}{ Butrecitrinadin (70) } & Cytotoxicity & MTT/HepG-2 & $78.57 \mu \mathrm{M}\left(\mathrm{IC}_{50}\right)$ & Curcumin $61.38 \mu \mathrm{M}\left(\mathrm{IC}_{50}\right)$ & [66] \\
\hline & & MTT/HL-60 & $60.31 \mu \mathrm{M}\left(\mathrm{IC}_{50}\right)$ & Curcumin $13.78 \mu \mathrm{M}\left(\mathrm{IC}_{50}\right)$ & [66] \\
\hline & & MTT/MCF-7 & $51.32 \mu \mathrm{M}\left(\mathrm{IC}_{50}\right)$ & Curcumin $20.68 \mu \mathrm{M}\left(\mathrm{IC}_{50}\right)$ & [66] \\
\hline & & MTT/LNCap & $32.94 \mu \mathrm{M}\left(\mathrm{IC}_{50}\right)$ & Curcumin $6.15 \mu \mathrm{M}\left(\mathrm{IC}_{50}\right)$ & [66] \\
\hline & Anti-malarial & $\begin{array}{l}\text { Flow cytometry/SYBR Green I fluorescence/ } P \text {. } \\
\text { falciparum chloroquine sensitive (3D7) }\end{array}$ & $>25.0 \mu \mathrm{M}\left(\mathrm{EC}_{50}\right)$ & Artesunate $0.074 \mu \mathrm{M}\left(\mathrm{EC}_{50}\right)$ & [66] \\
\hline \multirow[t]{3}{*}{ Cladosporitin B (74) } & Cytotoxicity & MTT/BEL-7042 & $29.4 \mu \mathrm{M}\left(\mathrm{IC}_{50}\right)$ & Adriamycin $11.9 \mu \mathrm{M}\left(\mathrm{IC}_{50}\right)$ & [67] \\
\hline & Cytotoxicity & MTT/K562 & $25.6 \mu \mathrm{M}\left(\mathrm{IC}_{50}\right)$ & Adriamycin $14.2 \mu \mathrm{M}\left(\mathrm{IC}_{50}\right)$ & [67] \\
\hline & Cytotoxicity & MTT/SGC-7901 & $41.7 \mu \mathrm{M}\left(\mathrm{IC}_{50}\right)$ & Adriamycin $6.66 \mu \mathrm{M}\left(\mathrm{IC}_{50}\right)$ & [67] \\
\hline \multirow[t]{2}{*}{ Talaroconvolutin A (76) } & Cytotoxicity & MTT/HeLa & $14.9 \mu \mathrm{M}\left(\mathrm{IC}_{50}\right)$ & Adriamycin $11.5 \mu \mathrm{M}\left(\mathrm{IC}_{50}\right)$ & [67] \\
\hline & Cytotoxicity & MTT/BEL-7042 & $26.7 \mu \mathrm{M}\left(\mathrm{IC}_{50}\right)$ & Adriamycin $11.9 \mu \mathrm{M}\left(\mathrm{IC}_{50}\right)$ & [67] \\
\hline Talaroconvolutin A (76) & $\alpha$-Glucosidase inhibitory & Glucose oxidase method & $78.2 \mu \mathrm{M}\left(\mathrm{IC}_{50}\right)$ & Acarbose $275.7 \mu \mathrm{M}\left(\mathrm{IC}_{50}\right)$ & [67] \\
\hline Cladosporamide A (77) & & TCPTP/Spectrophotometry & $54.0 \mu \mathrm{M}\left(\mathrm{IC}_{50}\right)$ & Oleanolic acid $0.8 \mu \mathrm{M}\left(\mathrm{IC}_{50}\right)$ & [68] \\
\hline \multirow[t]{4}{*}{ Cladosporilactam A (78) } & Cytotoxicity & MTT/HeLa & $0.76 \mu \mathrm{M}\left(\mathrm{IC}_{50}\right)$ & Adriamycin & [69] \\
\hline & & MTT/HT-29 & $2.48 \mu \mathrm{M}\left(\mathrm{IC}_{50}\right)$ & Adriamycin & [69] \\
\hline & & SRB/P388 & $1.35 \mu \mathrm{M}\left(\mathrm{IC}_{50}\right)$ & Adriamycin & [69] \\
\hline & & SRB/A549 & $3.11 \mu \mathrm{M}\left(\mathrm{IC}_{50}\right)$ & Adriamycin & [69] \\
\hline \multirow[t]{2}{*}{$\begin{array}{l}\text { 2-Methylacetate-3,5,6- } \\
\text { trimethylpyrazine } \\
\text { (84) }\end{array}$} & Insecticidal & $\mathrm{CM} /$ Helicoverpa armigera Hubner larvae & $100.0 \mu \mathrm{g} / \mathrm{mL}\left(\mathrm{IC}_{50}\right)$ & Azadirachtin $25.0 \mu \mathrm{g} / \mathrm{mL}\left(\mathrm{IC}_{50}\right)$ & [71] \\
\hline & Antimicrobial & Microplate assay/S. aureus & $12.5 \mu \mathrm{g} / \mathrm{mL}$ (MIC) & Ciprofloxacin $0.39 \mu \mathrm{g} / \mathrm{mL}$ (MIC) & [71] \\
\hline Cytochalasin D (85) & Antimicrobial & Microplate assay/S. aureus & $25.0 \mu \mathrm{g} / \mathrm{mL}(\mathrm{MIC})$ & Ciprofloxacin $0.39 \mu \mathrm{g} / \mathrm{mL}$ (MIC) & [71] \\
\hline \multirow[t]{2}{*}{ Pandangolide 3 (96) } & Antimicrobial & Microplate assay/C. glecosporioides & $2.0 \mu \mathrm{g} / \mathrm{mL}(\mathrm{MIC})$ & Amphotericin B 0.5 rg/mL (MIC) & [39] \\
\hline & & Microplate assay/B. sorokiniana & $8.0 \mu \mathrm{g} / \mathrm{mL}(\mathrm{MIC})$ & Amphotericin B 0.5 rg/mL (MIC) & [39] \\
\hline \multirow[t]{3}{*}{ Thiocladospolide A (101) } & Antimicrobial & Microplate assay/E. tarda & $1.0 \mu \mathrm{g} / \mathrm{mL}(\mathrm{MIC})$ & Chloramphenicol $0.5 \mu \mathrm{g} / \mathrm{mL}$ (MIC) & [39] \\
\hline & & Microplate assay/E. ictarda & $8.0 \mu \mathrm{g} / \mathrm{mL}(\mathrm{MIC})$ & Chloramphenicol $0.5 \mu \mathrm{g} / \mathrm{mL}$ (MIC) & [39] \\
\hline & & Microplate assay/C. glecosporioides & $2.0 \mu \mathrm{g} / \mathrm{mL}(\mathrm{MIC})$ & Amphotericin B $0.5 \mu \mathrm{g} / \mathrm{mL}$ (MIC) & [39] \\
\hline
\end{tabular}


Table 2. Cont

\begin{tabular}{|c|c|c|c|c|c|}
\hline Compound Name & Biological Activity & Assay, Organism, or Cell Line & Biological Results & Positive Control & Ref. \\
\hline \multirow[t]{3}{*}{ Thiocladospolide B (102) } & Antimicrobial & Microplate assay/C. glecosporioides & $2.0 \mu \mathrm{g} / \mathrm{mL}(\mathrm{MIC})$ & Amphotericin B 0.5 rg/mL (MIC) & [39] \\
\hline & & Microplate assay /P. piricola Nose & $32.0 \mu \mathrm{g} / \mathrm{mL}(\mathrm{MIC})$ & Amphotericin B 2.0 g/mL (MIC) & [39] \\
\hline & & Microplate assay/F. oxysporum f. sp. cucumerinum & $1.0 \mu \mathrm{g} / \mathrm{mL}(\mathrm{MIC})$ & Amphotericin B 0.5 g/mL (MIC) & [39] \\
\hline \multirow[t]{3}{*}{ Thiocladospolide C (103) } & Antimicrobial & Microplate assay/C. glecosporioides & $1.0 \mu \mathrm{g} / \mathrm{mL}(\mathrm{MIC})$ & Amphotericin B $0.5 \mu \mathrm{g} / \mathrm{mL}$ (MIC) & [39] \\
\hline & & Microplate assay /P. piricola Nose & $32.0 \mu \mathrm{g} / \mathrm{mL}(\mathrm{MIC})$ & Amphotericin B 2.0 g/mL (MIC) & [39] \\
\hline & & Microplate assay /F. oxysporum f. sp. cucumerinum & $32.0 \mu \mathrm{g} / \mathrm{mL}(\mathrm{MIC})$ & Amphotericin B $0.5 \mu \mathrm{g} / \mathrm{mL}$ (MIC) & [39] \\
\hline \multirow[t]{3}{*}{ Thiocladospolide D (104) } & Antimicrobial & Microplate assay / E. ictarda & $1.0 \mu \mathrm{g} / \mathrm{mL}(\mathrm{MIC})$ & Chloramphenicol $0.5 \mu \mathrm{g} / \mathrm{mL}$ (MIC) & [39] \\
\hline & & Microplate assay /P. piricola Nose & $32.0 \mu \mathrm{g} / \mathrm{mL}(\mathrm{MIC})$ & Amphotericin B $2.0 \mu \mathrm{g} / \mathrm{mL}$ (MIC) & [39] \\
\hline & & Microplate assay/F. oxysporum f. sp. cucumerinum & $1.0 \mu \mathrm{g} / \mathrm{mL}(\mathrm{MIC})$ & Amphotericin B $0.5 \mu \mathrm{g} / \mathrm{mL}$ (MIC) & [39] \\
\hline \multirow[t]{2}{*}{ Thiocladospolide F (106) } & Antimicrobial & Microplate assay/E. tarda & $2.0 \mu \mathrm{g} / \mathrm{mL}(\mathrm{MIC})$ & Chloramphenicol $0.5 \mu \mathrm{g} / \mathrm{mL}$ (MIC) & [79] \\
\hline & Antimicrobial & Microplate assay/H. maydis & $4.0 \mu \mathrm{g} / \mathrm{mL}(\mathrm{MIC})$ & Amphotericin B $0.5 \mu \mathrm{g} / \mathrm{mL}$ (MIC) & [79] \\
\hline Thiocladospolide G (108) & Antimicrobial & Microplate assay/E. tarda & $2.0 \mu \mathrm{g} / \mathrm{mL}(\mathrm{MIC})$ & Chloramphenicol $0.5 \mu \mathrm{g} / \mathrm{mL}$ (MIC) & [79] \\
\hline Thiocladospolide G (109) & Antimicrobial & Microplate assay/E. tarda & $4.0 \mu \mathrm{g} / \mathrm{mL}(\mathrm{MIC})$ & Chloramphenicol $1.0 \mu \mathrm{g} / \mathrm{mL}$ (MIC) & [78] \\
\hline Thiocladospolide H (110) & Antimicrobial & Microplate assay/E. ictarda & $8.0 \mu \mathrm{g} / \mathrm{mL}(\mathrm{MIC})$ & Chloramphenicol $1.0 \mu \mathrm{g} / \mathrm{mL}$ (MIC) & [78] \\
\hline Sporiolide A (113) & Cytotoxicity & MTT/L1210 & $0.13 \mu \mathrm{M}\left(\mathrm{IC}_{50}\right)$ & - & [80] \\
\hline \multirow[t]{8}{*}{ Dendrodolide A (117) } & Antimicrobial & Broth dilution assay/B. cereus & $12.5 \mu \mathrm{M}(\mathrm{MIC})$ & Ciprofloxacin $1.56 \mu \mathrm{M}(\mathrm{MIC})$ & [69] \\
\hline & & Broth dilution assay/T. halophilus & $3.13 \mu \mathrm{M}(\mathrm{MIC})$ & Ciprofloxacin $1.56 \mu \mathrm{M}(\mathrm{MIC})$ & [69] \\
\hline & & Broth dilution assay/S. epidermidis & $6.25 \mu \mathrm{M}(\mathrm{MIC})$ & Ciprofloxacin $0.78 \mu \mathrm{M}(\mathrm{MIC})$ & [69] \\
\hline & & Broth dilution assay/S. aureus & $6.25 \mu \mathrm{M}(\mathrm{MIC})$ & Ciprofloxacin $0.39 \mu \mathrm{M}(\mathrm{MIC})$ & [69] \\
\hline & & Broth dilution assay /E. coli & $12.5 \mu \mathrm{M}(\mathrm{MIC})$ & Ciprofloxacin $1.56 \mu \mathrm{M}(\mathrm{MIC})$ & [69] \\
\hline & & Broth dilution assay/P. putida & $12.5 \mu \mathrm{M}(\mathrm{MIC})$ & Ciprofloxacin $0.39 \mu \mathrm{M}(\mathrm{MIC})$ & [69] \\
\hline & & Broth dilution assay/N. brasiliensis & $6.25 \mu \mathrm{M}(\mathrm{MIC})$ & Ciprofloxacin $0.78 \mu \mathrm{M}(\mathrm{MIC})$ & [69] \\
\hline & & Broth dilution assay/V. parahaemolyticus & $12.5 \mu \mathrm{M}(\mathrm{MIC})$ & Ciprofloxacin $1.56 \mu \mathrm{M}(\mathrm{MIC})$ & [69] \\
\hline \multirow[t]{7}{*}{ Dendrodolide C (118) } & Antimicrobial & Broth dilution assay/B. cereus & $25.0 \mu \mathrm{M}(\mathrm{MIC})$ & Ciprofloxacin $1.56 \mu \mathrm{M}(\mathrm{MIC})$ & [69] \\
\hline & & Broth dilution assay /T. halophilus & $3.13 \mu \mathrm{M}(\mathrm{MIC})$ & Ciprofloxacin $1.56 \mu \mathrm{M}(\mathrm{MIC})$ & [69] \\
\hline & & Broth dilution assay/S. epidermidis & $25.0 \mu \mathrm{M}(\mathrm{MIC})$ & Ciprofloxacin $0.78 \mu \mathrm{M}$ (MIC) & [69] \\
\hline & & Broth dilution assay/S. aureus & $25.0 \mu \mathrm{M}(\mathrm{MIC})$ & Ciprofloxacin $0.39 \mu \mathrm{M}(\mathrm{MIC})$ & [69] \\
\hline & & Broth dilution assay/E. coli & $12.5 \mu \mathrm{M}(\mathrm{MIC})$ & Ciprofloxacin $1.56 \mu \mathrm{M}$ (MIC) & [69] \\
\hline & & Broth dilution assay/P. putida & $25.0 \mu \mathrm{M}(\mathrm{MIC})$ & Ciprofloxacin $0.39 \mu \mathrm{M}(\mathrm{MIC})$ & [69] \\
\hline & & Broth dilution assay/N. brasiliensis & $12.5 \mu \mathrm{M}(\mathrm{MIC})$ & Ciprofloxacin $0.78 \mu \mathrm{M}$ (MIC) & [69] \\
\hline
\end{tabular}


Table 2. Cont

\begin{tabular}{|c|c|c|c|c|c|}
\hline Compound Name & Biological Activity & Assay, Organism, or Cell Line & Biological Results & Positive Control & Ref. \\
\hline & & Broth dilution assay/V. parahaemolyticus & $25.0 \mu \mathrm{M}(\mathrm{MIC})$ & Ciprofloxacin $1.56 \mu \mathrm{M}$ (MIC) & [69] \\
\hline \multirow[t]{4}{*}{ Dendrodolide M (120) } & Antimicrobial & Broth dilution assay/B. cereus & $6.25 \mu \mathrm{M}(\mathrm{MIC})$ & Ciprofloxacin $1.56 \mu \mathrm{M}$ (MIC) & [69] \\
\hline & & Broth dilution assay/T. halophilus & $25.0 \mu \mathrm{M}(\mathrm{MIC})$ & Ciprofloxacin $1.56 \mu \mathrm{M}$ (MIC) & [69] \\
\hline & & Broth dilution assay/S. epidermidis & $25.0 \mu \mathrm{M}(\mathrm{MIC})$ & Ciprofloxacin $0.78 \mu \mathrm{M}$ (MIC) & [69] \\
\hline & & Broth dilution assay /S. aureus & $12.5 \mu \mathrm{M}(\mathrm{MIC})$ & Ciprofloxacin $0.39 \mu \mathrm{M}$ (MIC) & [69] \\
\hline \multirow[t]{4}{*}{ Dendrodolide C (118) } & Antimicrobial & Broth dilution assay/B. cereus & $25.0 \mu \mathrm{M}(\mathrm{MIC})$ & Ciprofloxacin $1.56 \mu \mathrm{M}(\mathrm{MIC})$ & [69] \\
\hline & & Broth dilution assay/E. coli & $25.0 \mu \mathrm{M}(\mathrm{MIC})$ & Ciprofloxacin $1.56 \mu \mathrm{M}(\mathrm{MIC})$ & [69] \\
\hline & & Broth dilution assay/N. brasiliensis & $25.0 \mu \mathrm{M}(\mathrm{MIC})$ & Ciprofloxacin $0.78 \mu \mathrm{M}(\mathrm{MIC})$ & [69] \\
\hline & & Broth dilution assay/V. parahaemolyticus & $25.0 \mu \mathrm{M}(\mathrm{MIC})$ & Ciprofloxacin $1.56 \mu \mathrm{M}$ (MIC) & [69] \\
\hline \multirow[t]{2}{*}{ Cladocladosin A (121) } & Antimicrobial & Microplate assay/E. tarda & $1.0 \mu \mathrm{g} / \mathrm{mL}(\mathrm{MIC})$ & Chloramphenicol $0.5 \mu \mathrm{g} / \mathrm{mL}$ (MIC) & [79] \\
\hline & Antimicrobial & Microplate assay/P. aeruginosa & $4.0 \mu \mathrm{g} / \mathrm{mL}(\mathrm{MIC})$ & Chloramphenicol $2.0 \mu \mathrm{g} / \mathrm{mL}$ (MIC) & [79] \\
\hline Ent-cladospolide F (123) & AchE inhibitory & $\begin{array}{l}\text { Modified Ellman's enzyme/Immunosorbent } \\
\text { assay }\end{array}$ & $40.26 \mu \mathrm{M}\left(\mathrm{IC}_{50}\right)$ & Tacrine $0.5 \mu \mathrm{M}\left(\mathrm{IC}_{50}\right)$ & [40] \\
\hline \multirow[t]{8}{*}{ Iso-cladospolide B (126) } & Antimicrobial & Broth dilution assay/B. cereus & $6.25 \mu \mathrm{M}(\mathrm{MIC})$ & Ciprofloxacin $1.56 \mu \mathrm{M}(\mathrm{MIC})$ & [69] \\
\hline & & Broth dilution assay/T. halophilus & $6.25 \mu \mathrm{M}(\mathrm{MIC})$ & Ciprofloxacin $1.56 \mu \mathrm{M}$ (MIC) & [69] \\
\hline & & Broth dilution assay/S. aureus & $25.0 \mu \mathrm{M}(\mathrm{MIC})$ & Ciprofloxacin $0.39 \mu \mathrm{M}(\mathrm{MIC})$ & [69] \\
\hline & & Broth dilution assay /E. coli & $25.0 \mu \mathrm{M}(\mathrm{MIC})$ & Ciprofloxacin $1.56 \mu \mathrm{M}(\mathrm{MIC})$ & [69] \\
\hline & & Broth dilution assay/P. putida & $6.25 \mu \mathrm{M}(\mathrm{MIC})$ & Ciprofloxacin $0.39 \mu \mathrm{M}$ (MIC) & [69] \\
\hline & & Broth dilution assay/N. brasiliensis & $12.5 \mu \mathrm{M}(\mathrm{MIC})$ & Ciprofloxacin $0.78 \mu \mathrm{M}(\mathrm{MIC})$ & [69] \\
\hline & & Broth dilution assay/V. parahaemolyticus & $25.0 \mu \mathrm{M}(\mathrm{MIC})$ & Ciprofloxacin $1.56 \mu \mathrm{M}$ (MIC) & [69] \\
\hline & & Microplate assay/C. mandshurica Miura & $8.0 \mu \mathrm{g} / \mathrm{mL}(\mathrm{MIC})$ & Nystatin $1.0 \mu \mathrm{g} / \mathrm{mL}$ (MIC) & [78] \\
\hline \multirow[t]{9}{*}{ Cladosporol C (143) } & Cytotoxicity & Trypan blue-cell viability assay/K562 & $>30.0 \mu \mathrm{M}\left(\mathrm{IC}_{50}\right)$ & Trichostatin A $0.24 \mu \mathrm{M}\left(\mathrm{IC}_{50}\right)$ & [82] \\
\hline & & Trypan blue-cell viability assay / A549 & $33.9 \mu \mathrm{M}\left(\mathrm{IC}_{50}\right)$ & Trichostatin A $0.05 \mu \mathrm{M}\left(\mathrm{IC}_{50}\right)$ & [82] \\
\hline & & Trypan blue-cell viability assay/Huh-7 & $>30.0 \mu \mathrm{M}\left(\mathrm{IC}_{50}\right)$ & Trichostatin A $0.08 \mu \mathrm{M}\left(\mathrm{IC}_{50}\right)$ & [82] \\
\hline & & Trypan blue-cell viability assay/H1975 & $45.6 \mu \mathrm{M}\left(\mathrm{IC}_{50}\right)$ & Trichostatin A $0.09 \mu \mathrm{M}\left(\mathrm{IC}_{50}\right)$ & [82] \\
\hline & & Trypan blue-cell viability assay/MCF-7 & $>30.0 \mu \mathrm{M}\left(\mathrm{IC}_{50}\right)$ & Trichostatin A $0.78 \mu \mathrm{M}\left(\mathrm{IC}_{50}\right)$ & [82] \\
\hline & & Trypan blue-cell viability assay/U937 & $>30.0 \mu \mathrm{M}\left(\mathrm{IC}_{50}\right)$ & Trichostatin A $0.06 \mu \mathrm{M}\left(\mathrm{IC}_{50}\right)$ & [82] \\
\hline & & Trypan blue-cell viability assay/BGC823 & $>30.0 \mu \mathrm{M}\left(\mathrm{IC}_{50}\right)$ & Trichostatin A $0.09 \mu \mathrm{M}\left(\mathrm{IC}_{50}\right)$ & [82] \\
\hline & & Trypan blue-cell viability assay/HL-60 & $72.5 \mu \mathrm{M}\left(\mathrm{IC}_{50}\right)$ & Trichostatin A $0.09 \mu \mathrm{M}\left(\mathrm{IC}_{50}\right)$ & [82] \\
\hline & & Trypan blue-cell viability assay / A549 & $>30.0 \mu \mathrm{M}\left(\mathrm{IC}_{50}\right)$ & Trichostatin A $0.11 \mu \mathrm{M}\left(\mathrm{IC}_{50}\right)$ & [82] \\
\hline
\end{tabular}


Table 2. Cont

\begin{tabular}{|c|c|c|c|c|c|}
\hline Compound Name & Biological Activity & Assay, Organism, or Cell Line & Biological Results & Positive Control & Ref. \\
\hline & & Trypan blue-cell viability assay/MOLT-4 & $14.4 \mu \mathrm{M}\left(\mathrm{IC}_{50}\right)$ & Trichostatin A $0.03 \mu \mathrm{M}\left(\mathrm{IC}_{50}\right)$ & [82] \\
\hline & & MTT/A549 & $14.0 \mu \mathrm{M}\left(\mathrm{IC}_{50}\right)$ & Cisplatin $1.3 \mu \mathrm{M}\left(\mathrm{IC}_{50}\right)$ & [84] \\
\hline & & MTT/HeLa & $4.0 \mu \mathrm{M}\left(\mathrm{IC}_{50}\right)$ & Paclitaxel $4.9 \mu \mathrm{M}\left(\mathrm{IC}_{50}\right)$ & [84] \\
\hline & Antimicrobial & Microplate assay/E. coli & $8.0 \mu \mathrm{g} / \mathrm{mL}(\mathrm{MIC})$ & Chloramphenicol $0.025 \mu \mathrm{g} / \mathrm{mL}$ (MIC) & [84] \\
\hline & & Microplate assay/M. luteus & $32.0 \mu \mathrm{g} / \mathrm{mL}(\mathrm{MIC})$ & Chloramphenicol $0.5 \mu \mathrm{g} / \mathrm{mL}$ (MIC) & [84] \\
\hline & & Microplate assay/V. harveyi & $16.0 \mu \mathrm{g} / \mathrm{mL}(\mathrm{MIC})$ & Chloramphenicol $2.0 \mu \mathrm{g} / \mathrm{mL}$ (MIC) & [84] \\
\hline & & Microplate assay/S. aureus & $6.25 \mu \mathrm{g} / \mathrm{mL}(\mathrm{MIC})$ & Ciprofloxacin $0.39 \mu \mathrm{g} / \mathrm{mL}$ (MIC) & [71] \\
\hline Cladosporol D (144) & Anti-inflammatory & $\begin{array}{l}\text { Spectrophotometry/Anti-COX-2/PGF } 2 \alpha \\
\text { inhibition }\end{array}$ & $60.2 \mu \mathrm{M}\left(\mathrm{IC}_{50}\right)$ & $\begin{array}{l}\text { Indomethacin } 18.3 \mu \mathrm{M}\left(\mathrm{IC}_{50}\right) \\
\mathrm{NS}-3981.0 \mu \mathrm{M}\left(\mathrm{IC}_{50}\right)\end{array}$ & [82] \\
\hline \multirow[t]{3}{*}{ Cladosporol E (145) } & Insecticidal & Measuring the corrected mortality $(\mathrm{CM})$ & $150.0 \mu \mathrm{g} / \mathrm{mL}\left(\mathrm{IC}_{50}\right)$ & Azadirachtin $25.0 \mu \mathrm{g} / \mathrm{mL}\left(\mathrm{IC}_{50}\right)$ & [71] \\
\hline & Antimicrobial & Microplate assay/S. aureus & $1.56 \mu \mathrm{g} / \mathrm{mL}(\mathrm{MIC})$ & Ciprofloxacin $0.39 \mu \mathrm{g} / \mathrm{mL}$ (MIC) & [71] \\
\hline & & Microplate assay/M. luteus & $12.5 \mu \mathrm{g} / \mathrm{mL}(\mathrm{MIC})$ & Ciprofloxacin $0.39 \mu \mathrm{g} / \mathrm{mL}$ (MIC) & [71] \\
\hline \multirow[t]{7}{*}{ Cladosporol F (146) } & Cytotoxicity & MTT/K562 & $23.0 \mu \mathrm{M}\left(\mathrm{IC}_{50}\right)$ & Doxorubicin $0.6 \mu \mathrm{M}\left(\mathrm{IC}_{50}\right)$ & [83] \\
\hline & & $\mathrm{SRB} / \mathrm{HeLa}$ & $13.8 \mu \mathrm{M}\left(\mathrm{IC}_{50}\right)$ & Doxorubicin $0.5 \mu \mathrm{M}\left(\mathrm{IC}_{50}\right)$ & [83] \\
\hline & & SRB/HCT-116 & $23.0 \mu \mathrm{M}\left(\mathrm{IC}_{50}\right)$ & Doxorubicin $0.2 \mu \mathrm{M}\left(\mathrm{IC}_{50}\right)$ & [83] \\
\hline & & MTT/HeLa & $10.0 \mu \mathrm{M}\left(\mathrm{IC}_{50}\right)$ & Paclitaxel $4.9 \mu \mathrm{M}\left(\mathrm{IC}_{50}\right)$ & [84] \\
\hline & Antimicrobial & Microplate assay/E. coli & $32.0 \mu \mathrm{g} / \mathrm{mL}(\mathrm{MIC})$ & Chloramphenicol $0.025 \mu \mathrm{g} / \mathrm{mL}$ (MIC) & [84] \\
\hline & & Microplate assay/M. luteus & $64.0 \mu \mathrm{g} / \mathrm{mL}(\mathrm{MIC})$ & chloramphenicol $0.5 \mu \mathrm{g} / \mathrm{mL}$ (MIC) & [84] \\
\hline & & Microplate assay/V. harveyi & $32.0 \mu \mathrm{g} / \mathrm{mL}$ (MIC) & Chloramphenicol $2.0 \mu \mathrm{g} / \mathrm{mL}$ (MIC) & [84] \\
\hline \multirow[t]{3}{*}{ Cladosporol G (147) } & Cytotoxicity & MTT/K562 & $8.8 \mu \mathrm{M}\left(\mathrm{IC}_{50}\right)$ & Doxorubicin $0.6 \mu \mathrm{M}\left(\mathrm{IC}_{50}\right)$ & [83] \\
\hline & & $\mathrm{SRB} / \mathrm{HeLa}$ & $3.9 \mu \mathrm{M}\left(\mathrm{IC}_{50}\right)$ & Doxorubicin $0.5 \mu \mathrm{M}$ (IC50) & [83] \\
\hline & & SRB/HCT-116 & $19.4 \mu \mathrm{M}\left(\mathrm{IC}_{50}\right)$ & Doxorubicin $0.2 \mu \mathrm{M}\left(\mathrm{IC}_{50}\right)$ & [83] \\
\hline \multirow[t]{8}{*}{ Cladosporol G (148) } & Cytotoxicity & MTT/A549 & $13.0 \mu \mathrm{M}\left(\mathrm{IC}_{50}\right)$ & Cisplatin $1.3 \mu \mathrm{M}\left(\mathrm{IC}_{50}\right)$ & [84] \\
\hline & & MTT/H446 & $11.0 \mu \mathrm{M}\left(\mathrm{IC}_{50}\right)$ & Adriamycin $4.0 \mu \mathrm{M}\left(\mathrm{IC}_{50}\right)$ & [84] \\
\hline & & MTT/Huh7 & $10.0 \mu \mathrm{M}\left(\mathrm{IC}_{50}\right)$ & Fluorouracil $6.2 \mu \mathrm{M}\left(\mathrm{IC}_{50}\right)$ & [84] \\
\hline & & MTT/L02 & $11.0 \mu \mathrm{M}\left(\mathrm{IC}_{50}\right)$ & Cisplatin $13.0 \mu \mathrm{M}\left(\mathrm{IC}_{50}\right)$ & [84] \\
\hline & & MTT/LM3 & $14.0 \mu \mathrm{M}\left(\mathrm{IC}_{50}\right)$ & Cisplatin $9.1 \mu \mathrm{M}\left(\mathrm{IC}_{50}\right)$ & [84] \\
\hline & & MTT/SW1990 & $15.0 \mu \mathrm{M}\left(\mathrm{IC}_{50}\right)$ & Gemcitabine $2.2 \mu \mathrm{M}\left(\mathrm{IC}_{50}\right)$ & [84] \\
\hline & Antimicrobial & Microplate assay/E. coli & $64.0 \mu \mathrm{g} / \mathrm{mL}(\mathrm{MIC})$ & Chloramphenicol $0.025 \mu \mathrm{g} / \mathrm{mL}$ (MIC) & [84] \\
\hline & & Microplate assay/M. luteus & $128.0 \mu \mathrm{g} / \mathrm{mL}(\mathrm{MIC})$ & Chloramphenicol $0.5 \mu \mathrm{g} / \mathrm{mL}$ (MIC) & [84] \\
\hline
\end{tabular}


Table 2. Cont

\begin{tabular}{|c|c|c|c|c|c|}
\hline Compound Name & Biological Activity & Assay, Organism, or Cell Line & Biological Results & Positive Control & Ref. \\
\hline & & Microplate assay/V. harveyi & $64.0 \mu \mathrm{g} / \mathrm{mL}(\mathrm{MIC})$ & 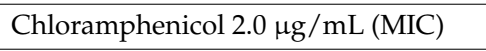 & [84] \\
\hline \multirow[t]{8}{*}{ Cladosporol H (149) } & Cytotoxicity & MTT/A549 & $5.0 \mu \mathrm{M}\left(\mathrm{IC}_{50}\right)$ & Cisplatin $1.3 \mu \mathrm{M}\left(\mathrm{IC}_{50}\right)$ & [84] \\
\hline & & MTT/H446 & $10.0 \mu \mathrm{M}\left(\mathrm{IC}_{50}\right)$ & Adriamycin $4.0 \mu \mathrm{M}\left(\mathrm{IC}_{50}\right)$ & [84] \\
\hline & & MTT/Huh7 & $1.0 \mu \mathrm{M}\left(\mathrm{IC}_{50}\right)$ & Fluorouracil $6.2 \mu \mathrm{M}\left(\mathrm{IC}_{50}\right)$ & [84] \\
\hline & & MTT/LM3 & $4.1 \mu \mathrm{M}\left(\mathrm{IC}_{50}\right)$ & Cisplatin $9.1 \mu \mathrm{M}\left(\mathrm{IC}_{50}\right)$ & [84] \\
\hline & & MTT/MCF-7 & $10.0 \mu \mathrm{M}\left(\mathrm{IC}_{50}\right)$ & Paclitaxel $1.8 \mu \mathrm{M}\left(\mathrm{IC}_{50}\right)$ & [84] \\
\hline & & MTT/SW1990 & $15.0 \mu \mathrm{M}\left(\mathrm{IC}_{50}\right)$ & Gemcitabine $2.2 \mu \mathrm{M}\left(\mathrm{IC}_{50}\right)$ & [84] \\
\hline & & Microplate assay/M. luteus & $64.0 \mu \mathrm{g} / \mathrm{mL}(\mathrm{MIC})$ & Chloramphenicol $0.5 \mu \mathrm{g} / \mathrm{mL}$ (MIC) & [84] \\
\hline & & Microplate assay/V. harveyi & $4.0 \mu \mathrm{g} / \mathrm{mL}(\mathrm{MIC})$ & Chloramphenicol $2.0 \mu \mathrm{g} / \mathrm{mL}$ (MIC) & [84] \\
\hline \multirow[t]{4}{*}{ Cladosporol I (150) } & Cytotoxicity & MTT/HeLa & $10.8 \mu \mathrm{M}\left(\mathrm{IC}_{50}\right)$ & Paclitaxel $4.9 \mu \mathrm{M}\left(\mathrm{IC}_{50}\right)$ & [84] \\
\hline & Antimicrobial & Microplate assay/E. coli & $64.0 \mu \mathrm{g} / \mathrm{mL}(\mathrm{MIC})$ & Chloramphenicol $0.025 \mu \mathrm{g} / \mathrm{mL}$ (MIC) & [84] \\
\hline & & Microplate assay/M. luteus & $64.0 \mu \mathrm{g} / \mathrm{mL}(\mathrm{MIC})$ & Chloramphenicol $0.5 \mu \mathrm{g} / \mathrm{mL}$ (MIC) & [84] \\
\hline & & Microplate assay/V. harveyi & $16.0 \mu \mathrm{g} / \mathrm{mL}(\mathrm{MIC})$ & Chloramphenicol $2.0 \mu \mathrm{g} / \mathrm{mL}$ (MIC) & [84] \\
\hline \multirow[t]{7}{*}{ Cladosporol J (151) } & & MTT/A549 & $15.0 \mu \mathrm{M}\left(\mathrm{IC}_{50}\right)$ & Cisplatin $1.3 \mu \mathrm{M}\left(\mathrm{IC}_{50}\right)$ & [84] \\
\hline & & MTT/H446 & $11.0 \mu \mathrm{M}\left(\mathrm{IC}_{50}\right)$ & Adriamycin $4.0 \mu \mathrm{M}\left(\mathrm{IC}_{50}\right)$ & [84] \\
\hline & & MTT/Huh7 & $20.0 \mu \mathrm{M}\left(\mathrm{IC}_{50}\right)$ & Fluorouracil $6.2 \mu \mathrm{M}\left(\mathrm{IC}_{50}\right)$ & [84] \\
\hline & & MTT/MCF-7 & $12.0 \mu \mathrm{M}\left(\mathrm{IC}_{50}\right)$ & Paclitaxel $1.8 \mu \mathrm{M}\left(\mathrm{IC}_{50}\right)$ & [84] \\
\hline & Antimicrobial & Microplate assay/E. coli & $16.0 \mu \mathrm{g} / \mathrm{mL}(\mathrm{MIC})$ & Chloramphenicol $0.025 \mu \mathrm{g} / \mathrm{mL}$ (MIC) & [84] \\
\hline & & Microplate assay/M. luteus & $64.0 \mu \mathrm{g} / \mathrm{mL}(\mathrm{MIC})$ & Chloramphenicol $0.5 \mu \mathrm{g} / \mathrm{mL}$ (MIC) & [84] \\
\hline & & Microplate assay/V. harveyi & $32.0 \mu \mathrm{g} / \mathrm{mL}(\mathrm{MIC})$ & 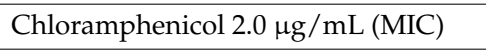 & [84] \\
\hline \multirow[t]{10}{*}{ Cladosporone A (152) } & Cytotoxicity & Trypan blue-cell viability assay/K562 & $14.3 \mu \mathrm{M}\left(\mathrm{IC}_{50}\right)$ & Trichostatin A $0.24 \mu \mathrm{M}\left(\mathrm{IC}_{50}\right)$ & [82] \\
\hline & & Trypan blue-cell viability assay / A549 & $15.7 \mu \mathrm{M}\left(\mathrm{IC}_{50}\right)$ & Trichostatin A $0.05 \mu \mathrm{M}\left(\mathrm{IC}_{50}\right)$ & [82] \\
\hline & & Trypan blue-cell viability assay/Huh-7 & $29.9 \mu \mathrm{M}\left(\mathrm{IC}_{50}\right)$ & Trichostatin A $0.08 \mu \mathrm{M}\left(\mathrm{IC}_{50}\right)$ & [82] \\
\hline & & Trypan blue-cell viability assay/H1975 & $40.6 \mu \mathrm{M}\left(\mathrm{IC}_{50}\right)$ & Trichostatin A $0.09 \mu \mathrm{M}\left(\mathrm{IC}_{50}\right)$ & [82] \\
\hline & & Trypan blue-cell viability assay/MCF-7 & $21.3 \mu \mathrm{M}\left(\mathrm{IC}_{50}\right)$ & Trichostatin A $0.78 \mu \mathrm{M}\left(\mathrm{IC}_{50}\right)$ & [82] \\
\hline & & Trypan blue-cell viability assay/U937 & $10.5 \mu \mathrm{M}\left(\mathrm{IC}_{50}\right)$ & Trichostatin A $0.06 \mu \mathrm{M}\left(\mathrm{IC}_{50}\right)$ & [82] \\
\hline & & Trypan blue-cell viability assay/BGC823 & $17.0 \mu \mathrm{M}\left(\mathrm{IC}_{50}\right)$ & Trichostatin A $0.09 \mu \mathrm{M}\left(\mathrm{IC}_{50}\right)$ & [82] \\
\hline & & Trypan blue-cell viability assay/HL-60 & $10.1 \mu \mathrm{M}\left(\mathrm{IC}_{50}\right)$ & Trichostatin A $0.09 \mu \mathrm{M}\left(\mathrm{IC}_{50}\right)$ & [82] \\
\hline & & Trypan blue-cell viability assay / A549 & $53.7 \mu \mathrm{M}\left(\mathrm{IC}_{50}\right)$ & Trichostatin A $0.11 \mu \mathrm{M}\left(\mathrm{IC}_{50}\right)$ & [82] \\
\hline & & Trypan blue-cell viability assay/MOLT-4 & $14.6 \mu \mathrm{M}\left(\mathrm{IC}_{50}\right)$ & Trichostatin A $0.03 \mu \mathrm{M}\left(\mathrm{IC}_{50}\right)$ & [82] \\
\hline
\end{tabular}


Table 2. Cont

\begin{tabular}{|c|c|c|c|c|c|}
\hline Compound Name & Biological Activity & Assay, Organism, or Cell Line & Biological Results & Positive Control & Ref. \\
\hline & Anti-inflammatory & $\begin{array}{l}\text { Spectrophotometry / Anti-COX-2/PGF } 2 \alpha \\
\text { inhibition }\end{array}$ & $49.1 \mu \mathrm{M}\left(\mathrm{IC}_{50}\right)$ & $\begin{array}{l}\text { Indomethacin } 18.3 \mu \mathrm{M}\left(\mathrm{IC}_{50}\right) \\
\text { NS-398 } 1.0 \mu \mathrm{M}\left(\mathrm{IC}_{50}\right)\end{array}$ & [82] \\
\hline \multirow[t]{2}{*}{ Aladothalen (160) } & Antimicrobial & Agar dilution method/B.cereus & $50.0 \mu \mathrm{M}(\mathrm{MIC})$ & Ciprofloxacin $<0.4 \mu \mathrm{M}$ (MIC) & [89] \\
\hline & & Agar dilution method/MRCNS & $25.0 \mu \mathrm{M}(\mathrm{MIC})$ & Ciprofloxacin $25.0 \mu \mathrm{M}$ (MIC) & [89] \\
\hline \multirow[t]{11}{*}{ Cladonaphchrom A (169) } & Antimicrobial & Microplate assay/S. albus & $1.25 \mu \mathrm{g} / \mathrm{mL}(\mathrm{MIC})$ & Ciprofloxacin $0.6 \mu \mathrm{g} / \mathrm{mL}$ (MIC) & [90] \\
\hline & & Microplate assay/E. coli & $2.5 \mu \mathrm{g} / \mathrm{mL}(\mathrm{MIC})$ & Ciprofloxacin $0.3 \mu \mathrm{g} / \mathrm{mL}$ (MIC) & [90] \\
\hline & & Microplate assay/B. subtilis & $10.0 \mu \mathrm{g} / \mathrm{mL}(\mathrm{MIC})$ & Ciprofloxacin $0.6 \mu \mathrm{g} / \mathrm{mL}$ (MIC) & [90] \\
\hline & & Microplate assay/M. tetragenus & $5.0 \mu \mathrm{g} / \mathrm{mL}(\mathrm{MIC})$ & Ciprofloxacin $0.3 \mu \mathrm{g} / \mathrm{mL}$ (MIC) & [90] \\
\hline & & Microplate assay/M. luteus & $10.0 \mu \mathrm{g} / \mathrm{mL}(\mathrm{MIC})$ & Ciprofloxacin $0.3 \mu \mathrm{g} / \mathrm{mL}$ (MIC) & [90] \\
\hline & & Microplate assay/A. brassicicola & $50.0 \mu \mathrm{g} / \mathrm{mL}(\mathrm{MIC})$ & Prochloraz $12.5 \mu \mathrm{g} / \mathrm{mL}$ (MIC) & [90] \\
\hline & & Microplate assay/P. parasitica var. nicotianae & $50.0 \mu \mathrm{g} / \mathrm{mL}(\mathrm{MIC})$ & Prochloraz $50.0 \mu \mathrm{g} / \mathrm{mL}$ (MIC) & [90] \\
\hline & & Microplate assay/C. capsici & $25.0 \mu \mathrm{g} / \mathrm{mL}(\mathrm{MIC})$ & Prochloraz 12.5 g/mL (MIC) & [90] \\
\hline & & Microplate assay/B. oryzae & $100.0 \mu \mathrm{g} / \mathrm{mL}$ (MIC) & Prochloraz $50.0 \mu \mathrm{g} / \mathrm{mL}$ (MIC) & [90] \\
\hline & & Microplate assay/D. medusaea & $50.0 \mu \mathrm{g} / \mathrm{mL}(\mathrm{MIC})$ & Prochloraz $50.0 \mu \mathrm{g} / \mathrm{mL}$ (MIC) & [90] \\
\hline & & Microplate assay/C. paradoxa & $50.0 \mu \mathrm{g} / \mathrm{mL}(\mathrm{MIC})$ & Prochloraz $25.0 \mu \mathrm{g} / \mathrm{mL}$ (MIC) & [90] \\
\hline \multirow{9}{*}{ Cladonaphchrom B (170) } & & Microplate assay/E. coli & $2.5 \mu \mathrm{g} / \mathrm{mL}$ (MIC) & Ciprofloxacin $0.3 \mu \mathrm{g} / \mathrm{mL}$ (MIC) & [90] \\
\hline & & Microplate assay/B. subtilis & $5.0 \mu \mathrm{g} / \mathrm{mL}(\mathrm{MIC})$ & Ciprofloxacin $0.6 \mu \mathrm{g} / \mathrm{mL}$ (MIC) & [90] \\
\hline & & Microplate assay/M. tetragenus & $5.0 \mu \mathrm{g} / \mathrm{mL}$ (MIC) & Ciprofloxacin $0.3 \mu \mathrm{g} / \mathrm{mL}$ (MIC) & [90] \\
\hline & & Microplate assay/M. luteus & $10.0 \mu \mathrm{g} / \mathrm{mL}(\mathrm{MIC})$ & Ciprofloxacin $0.3 \mu \mathrm{g} / \mathrm{mL}$ (MIC) & [90] \\
\hline & & Microplate assay/A. brassicicola & $25.0 \mu \mathrm{g} / \mathrm{mL}(\mathrm{MIC})$ & Prochloraz $12.5 \mu \mathrm{g} / \mathrm{mL}$ (MIC) & [90] \\
\hline & & Microplate assay/P. parasitica var. nicotianae & $50.0 \mu \mathrm{g} / \mathrm{mL}(\mathrm{MIC})$ & Prochloraz $50.0 \mu \mathrm{g} / \mathrm{mL}$ (MIC) & [90] \\
\hline & & Microplate assay/C. capsici & $25.0 \mu \mathrm{g} / \mathrm{mL}(\mathrm{MIC})$ & Prochloraz $12.5 \mu \mathrm{g} / \mathrm{mL}$ (MIC) & [90] \\
\hline & & Microplate assay/D. medusaea & $100.0 \mu \mathrm{g} / \mathrm{mL}$ (MIC) & Prochloraz $50.0 \mu \mathrm{g} / \mathrm{mL}$ (MIC) & [90] \\
\hline & & Microplate assay/C. paradoxa & $50.0 \mu \mathrm{g} / \mathrm{mL}(\mathrm{MIC})$ & Prochloraz $25.0 \mu \mathrm{g} / \mathrm{mL}$ (MIC) & [90] \\
\hline Malettinin A (178) & Antimicrobial & Microplate assay/T. rubrum & $33.1 \mu \mathrm{M}\left(\mathrm{IC}_{50}\right)$ & Clotrimazole $0.2 \mu \mathrm{M}\left(\mathrm{IC}_{50}\right)$ & [93] \\
\hline \multirow[t]{2}{*}{ Malettinin B (179) } & & Microplate assay/X. campestris & $28.3 \mu \mathrm{M}\left(\left(\mathrm{IC}_{50}\right)\right.$ & Chloramphenicol $2.1 \mu \mathrm{M}\left(\mathrm{IC}_{50}\right)$ & [93] \\
\hline & & Microplate assay/T. rubrum & $60.6 \mu \mathrm{M}\left(\mathrm{IC}_{50}\right)$ & Clotrimazole $0.2 \mu \mathrm{M}\left(\mathrm{IC}_{50}\right)$ & [93] \\
\hline \multirow[t]{2}{*}{ Malettinin C (180) } & Antimicrobial & Microplate assay/T. rubrum & $37.9 \mu \mathrm{M}\left(\mathrm{IC}_{50}\right)$ & Clotrimazole $0.2 \mu \mathrm{M}\left(\mathrm{IC}_{50}\right)$ & [93] \\
\hline & & Microplate assay/X. campestris & $83.2 \mu \mathrm{M}\left(\mathrm{IC}_{50}\right)$ & Chloramphenicol $2.1 \mu \mathrm{M}\left(\mathrm{IC}_{50}\right)$ & [93] \\
\hline Malettinin E (181) & Antimicrobial & Microplate assay/T. rubrum & $28.7 \mu \mathrm{M}\left(\mathrm{IC}_{50}\right)$ & Clotrimazole $0.2 \mu \mathrm{M}\left(\mathrm{IC}_{50}\right)$ & [93] \\
\hline
\end{tabular}


Table 2. Cont.

\begin{tabular}{|c|c|c|c|c|c|}
\hline Compound Name & Biological Activity & Assay, Organism, or Cell Line & Biological Results & Positive Control & Ref. \\
\hline & & Microplate assay/X. campestris & $30.7 \mu \mathrm{M}\left(\mathrm{IC}_{50}\right)$ & Chloramphenicol $2.1 \mu \mathrm{M}\left(\left(\mathrm{IC}_{50}\right)\right.$ & [93] \\
\hline Cladosporinone (182) & & Broth dilution assay/S. aureus & $64.0 \mu \mathrm{g} / \mathrm{mL}(\mathrm{MIC})$ & - & [94] \\
\hline Viriditoxin (183) & & Broth dilution assay /S. aureus & $\begin{array}{l}0.015 \mu \mathrm{g} / \mathrm{mL}(\mathrm{MIC}) \\
0.023 \mu \mathrm{M}(\mathrm{MIC})\end{array}$ & - & {$[94]$} \\
\hline Viriditoxin derivative 1 (184) & & Broth dilution assay/S. aureus & $2.0 \mu \mathrm{g} / \mathrm{mL}(\mathrm{MIC})$ & - & {$[94]$} \\
\hline Viriditoxin derivative 2 (185) & & Broth dilution assay/S. aureus & $16.0 \mu \mathrm{g} / \mathrm{mL}(\mathrm{MIC})$ & - & [94] \\
\hline $\begin{array}{l}\text { (2S,4S)-4-Methoxy-2-methylchroman-5- } \\
\text { ol } \\
\text { (201) }\end{array}$ & Antioxidant & DPPH assay & $5.66 \mu \mathrm{M}\left(\mathrm{IC}_{50}\right)$ & Ascorbic acid $3.29 \mu \mathrm{M}\left(\mathrm{IC}_{50}\right)$ & [95] \\
\hline (2S,4S)-2-Methylchroman-4,5-diol (203) & Antioxidant & DPPH assay & $6.67 \mu \mathrm{M}\left(\mathrm{IC}_{50}\right)$ & Ascorbic acid $3.29 \mu \mathrm{M}\left(\mathrm{IC}_{50}\right)$ & [95] \\
\hline \multirow[t]{4}{*}{ Citrinin H1 (206) } & Insecticidal & Measuring the corrected mortality $(\mathrm{CM})$ & $100.0 \mu \mathrm{g} / \mathrm{mL}\left(\mathrm{IC}_{50}\right)$ & Azadirachtin $25.0 \mu \mathrm{g} / \mathrm{mL}\left(\mathrm{IC}_{50}\right)$ & {$[71]$} \\
\hline & Antimicrobial & Microplate assay/S. aureus & $6.25 \mu \mathrm{g} / \mathrm{mL}(\mathrm{MIC})$ & Ciprofloxacin $0.39 \mu \mathrm{g} / \mathrm{mL}$ (MIC) & {$[71]$} \\
\hline & & Microplate assay/E. coli & $12.5 \mu \mathrm{g} / \mathrm{mL}$ (MIC) & Ciprofloxacin $0.19 \mu \mathrm{g} / \mathrm{mL}$ (MIC) & [71] \\
\hline & & Microplate assay/B. cereus & $12.5 \mu \mathrm{g} / \mathrm{mL}$ (MIC) & Ciprofloxacin $0.19 \mu \mathrm{g} / \mathrm{mL}$ (MIC) & {$[71]$} \\
\hline \multirow[t]{3}{*}{ Vermistatin (214) } & Insecticidal & CM/Helicoverpa armigera Hubner larvae & $150.0 \mu \mathrm{g} / \mathrm{mL}\left(\mathrm{IC}_{50}\right)$ & Azadirachtin $25.0 \mu \mathrm{g} / \mathrm{mL}\left(\mathrm{IC}_{50}\right)$ & [71] \\
\hline & Antimicrobial & Microplate assay/S. aureus & $25.0 \mu \mathrm{g} / \mathrm{mL}(\mathrm{MIC})$ & Ciprofloxacin $0.39 \mu \mathrm{g} / \mathrm{mL}$ (MIC) & {$[71]$} \\
\hline & & Microplate assay/B. cereus & $25.0 \mu \mathrm{g} / \mathrm{mL}(\mathrm{MIC})$ & Ciprofloxacin $0.39 \mu \mathrm{g} / \mathrm{mL}$ (MIC) & [71] \\
\hline 3-Phenyl-2-propenoic acid (232) & Insecticidal & Inhibitinon $50 \%$ / B. amphitrite & $84.28 \mu \mathrm{g} / \mathrm{mL}\left(\mathrm{EC}_{50}\right)$ & FSW with DMSO & {$[60]$} \\
\hline \multirow[t]{5}{*}{ Cladosporinone (182) } & & Broth dilution assay/S. aureus & $64.0 \mu \mathrm{g} / \mathrm{mL}(\mathrm{MIC})$ & - & {$[94]$} \\
\hline & & Lethality $50 \% / B$. amphitrite & $>200 \mu \mathrm{g} / \mathrm{mL}\left(\mathrm{LC}_{50}\right)$ & FSW with DMSO & {$[60]$} \\
\hline & & Inhibitinon 50\%/B. neritina & $11.15 \mu \mathrm{g} / \mathrm{mL}\left(\mathrm{EC}_{50}\right)$ & FSW with DMSO & {$[60]$} \\
\hline & & Lethality $50 \% / B$. neritina & $>200 \mu \mathrm{g} / \mathrm{mL}\left(\mathrm{LC}_{50}\right)$ & FSW with DMSO & {$[60]$} \\
\hline & Antimicrobial & Serial dilution/L. hongkongensis & $80 \mu \mathrm{g} / \mathrm{mL}(\mathrm{MIC})$ & $\begin{array}{l}\text { Streptomycin } 250 \mu \mathrm{g} / \mathrm{mL} \text { (MIC) } \\
\text { Penicillin } 0.25 \mu \mathrm{g} / \mathrm{mL} \text { (MIC) }\end{array}$ & {$[60]$} \\
\hline $\begin{array}{l}\text { 3-(2,3-Dihydroxy phenoxy) butanoic } \\
\text { acid (233) }\end{array}$ & Antioxidant & DPPH assay & $0.24 \mu \mathrm{M}\left(\mathrm{IC}_{50}\right)$ & Ascorbic acid $3.29 \mu \mathrm{M}\left(\mathrm{IC}_{50}\right)$ & [95] \\
\hline $\begin{array}{l}\text { Methyl (3S)-3-(2,3-dihydroxy } \\
\text { phenyloxy)butanoate (235) }\end{array}$ & Antioxidant & DPPH assay & $2.65 \mu \mathrm{M}\left(\mathrm{IC}_{50}\right)$ & Ascorbic acid $3.29 \mu \mathrm{M}\left(\mathrm{IC}_{50}\right)$ & [95] \\
\hline \multirow[t]{4}{*}{ 2-Phenylethanol (238) } & Insecticidal & Inhibitinon $50 \% / B$. amphitrite & $53.65 \mu \mathrm{g} / \mathrm{mL}\left(\mathrm{EC}_{50}\right)$ & FSW with DMSO & {$[60]$} \\
\hline & & Lethality $50 \% /$ B. amphitrite & $>200 \mu \mathrm{g} / \mathrm{mL}\left(\mathrm{LC}_{50}\right)$ & FSW with DMSO & {$[60]$} \\
\hline & & Inhibitinon 50\%/B. neritina & $102.23 \mu \mathrm{g} / \mathrm{mL}\left(\mathrm{EC}_{50}\right)$ & FSW with DMSO & {$[60]$} \\
\hline & & Lethality $50 \%$ / B. neritina & $>200 \mu \mathrm{g} / \mathrm{mL}\left(\mathrm{LC}_{50}\right)$ & FSW with DMSO & {$[60]$} \\
\hline
\end{tabular}


Table 2. Cont.

\begin{tabular}{|c|c|c|c|c|c|}
\hline Compound Name & Biological Activity & Assay, Organism, or Cell Line & Biological Results & Positive Control & Ref. \\
\hline $\begin{array}{l}\text { 4-O- } \alpha \text {-D-Ribofuranose-2-pentyl-3- } \\
\text { phemethylol } \\
(\mathbf{2 4 0 )}\end{array}$ & $\alpha$-Glucosidase inhibitory & Colorimetric assay & $2.05 \mu \mathrm{M}\left(\mathrm{IC}_{50}\right)$ & Acarbose $2.35 \mu \mathrm{M}\left(\mathrm{IC}_{50}\right)$ & [97] \\
\hline Cladosporin D (247) & Antioxidant & DPPH assay & $16.4 \mu \mathrm{M}\left(\mathrm{IC}_{50}\right)$ & Ascorbic acid $4.9 \mu \mathrm{M}\left(\mathrm{IC}_{50}\right)$ & [36] \\
\hline \multirow[t]{2}{*}{$\begin{array}{l}\text { (2S)-7,4'-dihydroxy-5-methoxy-8- }(\gamma, \gamma- \\
\text { dimethylallyl)-flavanone } \\
\text { (248) }\end{array}$} & $\begin{array}{l}\text { Protein tyrosine } \\
\text { phosphatase 1B } \\
\text { inhibitory }\end{array}$ & PTP1B/Spectrophotometry & $11.0 \mu \mathrm{M}\left(\mathrm{IC}_{50}\right)$ & Oleanolic acid $0.9 \mu \mathrm{M}\left(\mathrm{IC}_{50}\right)$ & [68] \\
\hline & & TCPTP/Spectrophotometry & $27.0 \mu \mathrm{M}\left(\mathrm{IC}_{50}\right)$ & Oleanolic acid $0.8 \mu \mathrm{M}\left(\mathrm{IC}_{50}\right)$ & [68] \\
\hline \multirow{3}{*}{ Bis(2-ethylhexyl)phthalate (249) } & & Lethality $50 \% /$ B. amphitrite & $>200 \mu \mathrm{g} / \mathrm{mL}\left(\mathrm{LC}_{50}\right)$ & FSW with DMSO & [60] \\
\hline & & Inhibitinon 50\%/B. neritina & $77.85 \mu \mathrm{g} / \mathrm{mL}\left(\mathrm{EC}_{50}\right)$ & FSW with DMSO & [60] \\
\hline & & Lethality $50 \% / B$. neritina & $>200 \mu \mathrm{g} / \mathrm{mL}\left(\mathrm{LC}_{50}\right)$ & FSW with DMSO & [60] \\
\hline \multirow[t]{4}{*}{ 1,1'-Dioxine-2,2'-dipropionic acid (252) } & Insecticidal & $\begin{array}{l}\text { Measuring the corrected mortality } \\
\text { (CM)/Helicoverpa armigera Hubner larvae }\end{array}$ & $150.0 \mu \mathrm{g} / \mathrm{mL}\left(\mathrm{IC}_{50}\right)$ & Azadirachtin 25.0 rg/mL ( $\left.\mathrm{IC}_{50}\right)$ & {$[70,71]$} \\
\hline & Antimicrobial & Microplate assay/S. aureus & $25.0 \mu \mathrm{g} / \mathrm{mL}(\mathrm{MIC})$ & Ciprofloxacin $0.39 \mu \mathrm{g} / \mathrm{mL}$ (MIC) & [71] \\
\hline & & Microplate assay/E. coli & $25.0 \mu \mathrm{g} / \mathrm{mL}(\mathrm{MIC})$ & Ciprofloxacin $0.19 \mu \mathrm{g} / \mathrm{mL}$ (MIC) & [71] \\
\hline & & Microplate assay/B. cereus & $12.5 \mu \mathrm{g} / \mathrm{mL}(\mathrm{MIC})$ & Ciprofloxacin $0.39 \mu \mathrm{g} / \mathrm{mL}$ (MIC) & [71] \\
\hline $\begin{array}{l}3 \beta, 5 \alpha \text {-Dihydroxy- } 6 \beta \text {-methoxyergosta- } \\
\text { 7,22-diene } \\
\text { (262) }\end{array}$ & Antiviral & Neuraminidase inhibition assay/RSV & $0.11 \mu \mathrm{M}\left(\mathrm{IC}_{50}\right)$ & Ribavirin $0.08 \mu \mathrm{M}\left(\mathrm{IC}_{50}\right)$ & [100] \\
\hline $\begin{array}{l}5 \alpha, 8 \alpha \text {-Epidioxy-ergosta-6,9,22E-triene- } \\
3 \beta \text {-ol } \\
(258)\end{array}$ & Antiviral & Neuraminidase inhibition assay/RSV & $0.17 \mu \mathrm{M}\left(\mathrm{IC}_{50}\right)$ & Ribavirin $0.08 \mu \mathrm{M}\left(\mathrm{IC}_{50}\right)$ & [100] \\
\hline \multirow[t]{2}{*}{$\begin{array}{l}\text { 3 } \alpha \text {-Hydroxy-pregna-7-ene-6,20-dione }= \\
\text { Cladosporisteroid B (268) }\end{array}$} & Antiviral & Neuraminidase inhibition assay/RSV & $0.12 \mu \mathrm{M}\left(\mathrm{IC}_{50}\right)$ & Ribavirin $0.08 \mu \mathrm{M}\left(\mathrm{IC}_{50}\right)$ & [100] \\
\hline & & CPE inhibition assay/ Influenza A H3N3 virus & $16.2 \mu \mathrm{M}\left(\mathrm{IC}_{50}\right)$ & Oseltamivir $34.0 \mathrm{nM}\left(\mathrm{IC}_{50}\right)$ & [61] \\
\hline $\begin{array}{l}\text { (3E,8E,6S)-Undeca-3,8,10-trien-1,6-diol } \\
(\mathbf{2 8 6})\end{array}$ & Cytotoxicity & SRB/H1975 & $10.0 \mu \mathrm{M}\left(\mathrm{IC}_{50}\right)$ & Adriamycin $0.38 \mu \mathrm{M}\left(\mathrm{IC}_{50}\right)$ & [95] \\
\hline
\end{tabular}


Pectinases are hydrolytic enzymes that are accountable for the hydrolysis of pectins. They are commonly found in fungi, bacteria, and plants. They have remarkable importance in the food industry such as vegetables and fruits processing, wine production, and olive oil extraction, as well as coffee, cocoa, and tea fermentation. They are utilized in the beverage industry to produce high yields due to improving clarification and pressing of concentrated fruit juices [103]. Bastos et al. purified pectinase enzymes PG and PME from C. cladosporioides using the Buescher and Furmanski procedure after 10-day incubation and precipitation with $\left(\mathrm{NH}_{4}\right)_{2} \mathrm{SO}_{4}$ and benzoate buffer at $\mathrm{pH} 4.0$ [49].

Agarases and carrageenases can decompose algal biomass, producing carrageenans and agars that are the major components of the red algae cell wall. Furthermore, agarases hydrolyze agar, resulting in oligosaccharides that are employed as food additives with beneficial influences on human health $[104,105]$. Additionally, carrageenases are used to obtain carrageenans that have varied industrial applications as emulsifying, thickening, and gelling agents in the preparation of food, as well as bioactivities such as anti-tumor, antiviral, antithrombotic, immunomodulatory, anticoagulant, and antioxidant [106]. Cladosporium sp. isolated from the Antarctic macroalgae Ascoseira mirabilis and Georgiella confuens produced agarase that may have industrial importance in the extraction of agar or its byproducts such as bioactive galactose and oligosaccharides exist in the algal biomass to be utilized as substrates of 3rd generation bioethanol [11].

Xylan, the main component of hemicelluloses in the plant cell walls, represents about one-third of all renewable organic carbon on earth. Xylanases hydrolyze xylan to oligosaccharides that are further degraded to xylose. The latter is utilized for xylitol and bioethanol production. Xylanases have remarkable biotechnological influence in developing ecofriendly technologies in the pulp and paper industry and in food and feed industries, and for generating chemicals and liquid fuels from lignocellulose [107-109]. The coldactive xylanases have notable applications in bioremediation and food and textile and industries [110]. Cladosporium sp. isolated from Antarctic marine sponge had high xylanase potential when grown on wheat bran and pure xylans at lower temperatures that is a feature of cold-active enzymes [48]. Therefore, cold-active xylanases preparations from Cladosporium sp. could be convenient for many biotechnological processes, utilizing moderate- to low-temperature processes, especially those in food industries [48]. Gil-Durán et al. purified and characterized $X y n A$, a cold-active endo-xylanase from Cladosporium sp. derived from Antarctic sponge. XynA is highly active on xylans with high arabinose content. Moreover, it is the most thermolabile endo-xylanase reported from filamentous fungus. Therefore, it could be a good alternative in some biotechnological operations to avoid heating, thereby reducing the costs [45].

The three main lignin-hydrolyzing enzymes that have great potential for industrial applications are LiP (lignin peroxidase), MnP (manganese-dependent peroxidase), and Lac (laccase) [111]. LiP is a high oxidant heme protein that oxidizes non-phenolic and phenolic substrates. $\mathrm{MnP}$ is a $\mathrm{H}_{2} \mathrm{O}_{2}$-dependent glycoprotein that needs $\mathrm{Mn}^{2+}$ for oxidizing aromatic dyes and mono-aromatic phenols [112]. Laccase is multi-copper oxidase, which oxidizes aromatic amines and catalyzes the $\mathrm{O}_{2}$ reduction to $\mathrm{H}_{2} \mathrm{O}$ [111]. C. cladosporioides CBMAI 857 isolated from the Brazilian cnidarian Palythoa variabilis produced ligninolytic enzymes ( $\mathrm{LiP}, \mathrm{MnP}$, and Lac) with particular response to the various conditions of salinity and carbon sources. It possessed high values of $\mathrm{MnP}$ and laccase activities under salinity ( $12.5 \%$ and $23 \% w / v$, respectively), indicating the potential use of this fungus for industrial applications and bioremediation of high-salt contaminated sites [50].

RBBR (Remazol Brilliant Blue R) and polymeric dyes decolorization has been assigned as an effective screening method for the fungi ability to degrade recalcitrant pollutants, including aromatic compounds such as PAHs. It was demonstrated that marine-derived fungi are often more effective than terrestrial fungi in treating various colored effluents because they are better adapted to perform under extreme conditions such as high salinity [113]. C. cladosporioides CBMAI-857 associated with the coral Palythoa caribaeorum was 
tested for its RBBR decolorizing potential. It had efficient dye decolorization potential (93\%) after 12 days in both liquid and solid media [114]. Further, Cladosporium sp. associated with the seagrass Posidonia oceanica produced tannases and ligninolytic enzymes at high salt concentrations. Its laccase and peroxidase activity was evident by the degradation of RBBR and Amaranth Red dyes [12,115].

Invertase is a $\beta$-fructo-furanosidase that catalyzes sucrose conversion into fructose and glucose, giving invert syrup. This invert syrup is utilized in the beverage and food industries as a humectant in non-crystallizing creams, candies, artificial honey, and jam preparation [116]. Molasses is a sugar solution that is obtained as a co-product of sugar production. Due to its high sucrose content and low cost, it is utilized as an invertase production substrate to produce industrially valuable substances [117]. However, it contains melanoidins, which are dark brown pigments. Its discharge in the soil prohibits seed germination and decreases manganese availability and soil alkalinity. Furthermore, it blocks photosynthesis and sunlight penetration in the aquatic system [118]. Therefore, its removal from molasses-based wastewater is potentially important for environmental safety. Taskin et al. reported that $C$. herbarum ER-25 possessed a high invertase potential and removed melanoidins from molasses through bio-adsorption and biodegradation mechanisms by Lac and $\mathrm{MnP}$ in the non-sterilized medium than in sterilized one at $5.5 \mathrm{pH}$ and $20^{\circ} \mathrm{C}$. Therefore, this cold-adapted fungus can be used for molasses de-colorization [46].

Cellulose is a main component of the plant material that is abundantly utilized for the production of alternative liquid fuels such as bioethanol. C. sphaerospermum obtained from deteriorated seaweed Ulva through SSF (solid-state fermentation) produced cellulase that had saccharification potential of seaweed biomass using green seaweed Ulva fasciata. Therefore, this cellulase can be utilized for saccharification of cellulosic feedstock for bioethanol production from marine macro-algal feedstock [47].

Biocatalysis is an eco-friendly process for renewable raw materials and clean energy production and for the remediation of environmental contaminants [119]. Recently, the synthesis of industrial and chemically interesting complex molecules using biocatalysts, including enzymes and whole-cell systems is a grown-research field. Reductases have been utilized for various substrates reduction such as aldehydes, carboxylic acid derivatives, ketones, nitro compounds, and nitriles [119,120]. Furthermore, it has been reported that microorganisms' whole cells are a potential source for new enzymes used in carbonylated compounds reduction [121]. Knoevenagel condensation is a very useful synthetic tool for functionalization, as well as for increasing the carbon chains that is applied for the synthesis of intermediates polymers and various bioactive organic compounds [122]. Birolli et al. reported that the bio-reduction of Knoevenagel adducts between cyanoacetamide and aromatic aldehydes was achieved in considerable yields with whole-cells of Cladosporium sp. CBMAI 1237 isolated from Dragmacidon reticulatum, revealing the existence of ene-reductases [43]. Additionally, C. cladosporioides CBMAI-857 isolated from the Brazilian cnidarian Palythoa caribaeorum catalyzed the asymmetric bio-reduction of 1-(4-methoxyphenyl)ethanone to 1-(4-methoxyphenyl)ethanol [123]. Moreover, the spongeassociated C. cladosporioides CBMAI-857 catalyzed the enantio-selective bio-reduction of different aromatic ketones at $\mathrm{pH} 7.0$ and $32{ }^{\circ} \mathrm{C}$ [124].

\section{Secondary Metabolites and Bioactivities of Marine-Associated Cladosporium Species}

Marine-associated Cladosporium species are rich with diverse types of metabolites with varied structural features such as macrolides, fatty acids, pyrones, phenolics, alkaloids, diketopiperazines, terpenes, sterols, quinones, lactones, and tetramic acid derivatives. Their classification was carried out here according to the chemical nature. During our search, it was found that some of the reported metabolites had the same structures and molecular formulae with different nomenclature. On the other hand, some metabolites had the same names with different structures. Moreover, some metabolites did not have names, thus they are named here using the AUPAC system for nomenclature. Herein, the reported 
secondary metabolites from Cladosporium species, as well as their bioactivities have been discussed (Tables 1 and 2).

\subsection{Tetramic Acid Derivatives}

Tetramic acids are five-membered heterocycles with a pyrrolidine-2,4-dione core that are formed by the fusion of polyketide units and amino acid [125]. The tertarmic acid moiety is commonly present as 3-acyl or 4-O-alkyl ether derivatives [126]. These structures can be characterized as simple heterocycles or more complex systems possibly containing long chains or fused polycyclic skeletons [127]. They are found in varied natural metabolites and isolated from various terrestrial and marine species, such as bacteria, sponges, and fungi $[127,128]$. They exhibited a wide range of bioactivities: cytotoxic, antimicrobial, antiulcer, and antiviral [125]. Note that 30 tetramic acid derivatives have been reported from marine-derived Cladosporium species, 28 (93.3\%) of them are from C. sphaerospermum.

The tetramic acid derivatives, cladosins A, B, D, and E (1, 2, 4, and 5) biosynthesized by C. sphaerospermum 2005-01-E3 obtained from deep-sea sludge had no activity towards influenza A H1N1 virus (Figure 1). While 3 exhibited anti-H1N1 activity $\left(\mathrm{IC}_{50}\right.$ $276.0 \mu \mathrm{M})$ in comparison to ribavirin $\left(\mathrm{IC}_{50} 131.0 \mu \mathrm{M}\right)$ [42]. Moreover, they showed no NF- $\mathrm{BB}$ inhibitory and no cytotoxic effect towards BGC-823, HL-60, HCT-8, A2780, A549, and Bel-7402 cell lines, as well as no activity towards Mycobacterium tuberculosis in the disk diffusion method [42]. Moreover, cladosins B (2), C (3), F (5), and L (11) separated from C. sphaerospermum SW67 associated with Hydractinia echinat hydroid polyp were assessed for protection towards cisplatin-caused cell damage in LLC-PK1 cells [53]. The co-treatment with compounds 2 and 5 alleviated the LLC-PK1 cells damage induced by cisplatin (Conc. $25 \mu \mathrm{M}$ ). Compound 2 (Conc. $100 \mu \mathrm{M}$ ) recovered cell viability with $90.68 \%$ that was more than NAC ( $N$-acetylcysteine, $88.23 \%$, Conc. $500 \mu \mathrm{M}$ ), whereas 5 (Conc. 50 and $100 \mu \mathrm{M}$ ) increased cell viability by 77.65 and $85.60 \%$, respectively. Thus, 2 may be a candidate for treating cisplatin-produced unwanted effects and/or to prohibited nephrotoxicity induced by anticancer drugs. It was proposed that the existence of the C- 8 hydroxy group may be essential for the reno-protective effect towards cisplatin-produced toxicity in LLC-PK1 cells [53].

In 2015, by OSMAC (one strain many compounds) technique, Yu et al. separated compounds 5 and 6 from C. sphaerospermum 2005-01-E3 that did not have anti-influenza A H1N1, anticancer, and anti-tubercular, as well as no NF-kB inhibitory activities [54]. Note that a tetramic acid derivative named cladosin $\mathrm{L}$ with a different structure was separated in 2020 by Pan et al. from the plant-associated C. sphaerospermum WBS017 isolated from Fritillaria unibracteata var. wabuensis [14]. Cladosins H-K (7-10) and cladodionen (13) were isolated from sediment-derived C. sphaerospermum L3P3 and evaluated for cytotoxic capacity towards PC-3, MGC-803, SH-SY5Y, and HCT-116 cell lines using SRB method and against K562 and HL-60 using MTT method (Figure 2). Compounds 8-10 and 13 had a cytotoxic effect against $\mathrm{HL}-60$ and $\mathrm{K} 562$ cell lines with $\mathrm{IC}_{50}$ ranging from 2.8 to $7.8 \mu \mathrm{M}$, while $7\left(\mathrm{IC}_{50}>10 \mu \mathrm{M}\right)$ was inactive. The results revealed that the $\mathrm{C}-8$ absolute configuration and aniline moiety were essential for activity [55] (Table 2).

C. sphaerospermum SW67 associated with marine invertebrate Hydractinia echinata yielded three new spirocyclic tetramic acid-related metabolites 12, 29, and 30 (Figure 3). Compound 12 has a tetramic acid moiety conjugated with an unprecedented 2,7-diazaspiro[4.5]decane1,4-dione core one, while 29 and 30 are tetramic acid stereoisomers with a C-3 quaternary center, bearing a six-membered lactone ring and a trans-hexylenic alcohol side chain. These metabolites had weak inhibitory effects versus HCC70, Bt549, MDA-MB-468, and MDAMB-231 in the SRB bioassay $\left(\mathrm{IC}_{50}\right.$ ranged from 70 to $\left.85 \mu \mathrm{M}\right)$, compared to etoposide $\left(\mathrm{IC}_{50}\right.$ ranged from 1.76 to $2.27 \mu \mathrm{M}$ ) [38]. 
<smiles>CO[C@@H](C/C(C)=C1\NC(=O)/C(=C(\N)[C@@H](C/C(N)=C2\C(=O)NC(=C(C)C)C2=O)OC)C1=O)C[C@H](C)O</smiles>

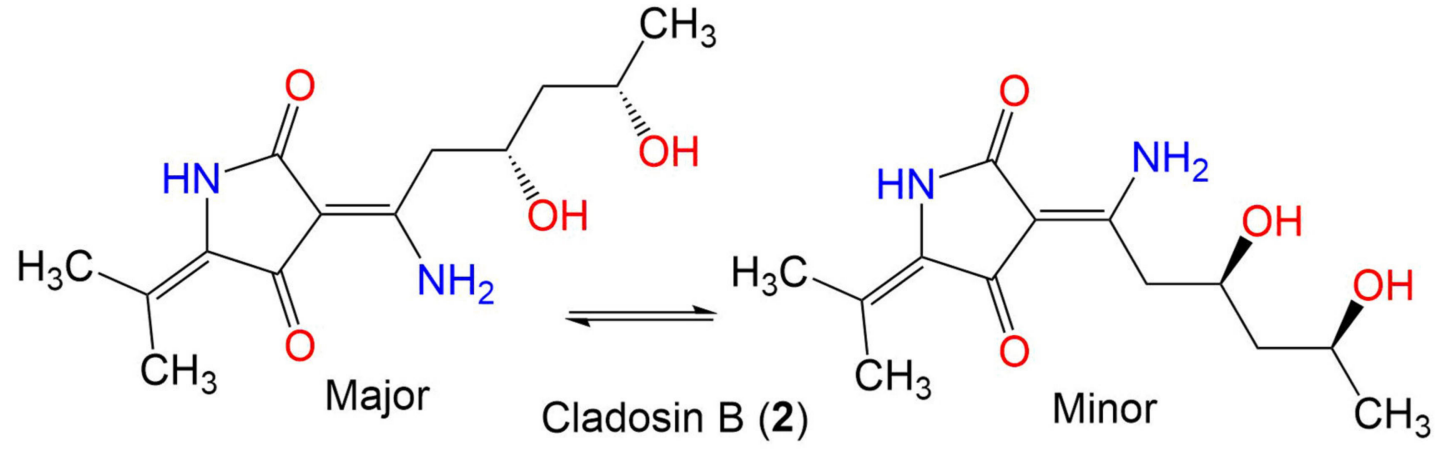<smiles>CC(C)=C1NC(=O)C(=C(N)C=CC[C@@H](C)O)C1=O</smiles><smiles>CC(C)=C1NC(=O)C(=C(N)/C=C\CC(C)O)C1=O</smiles>

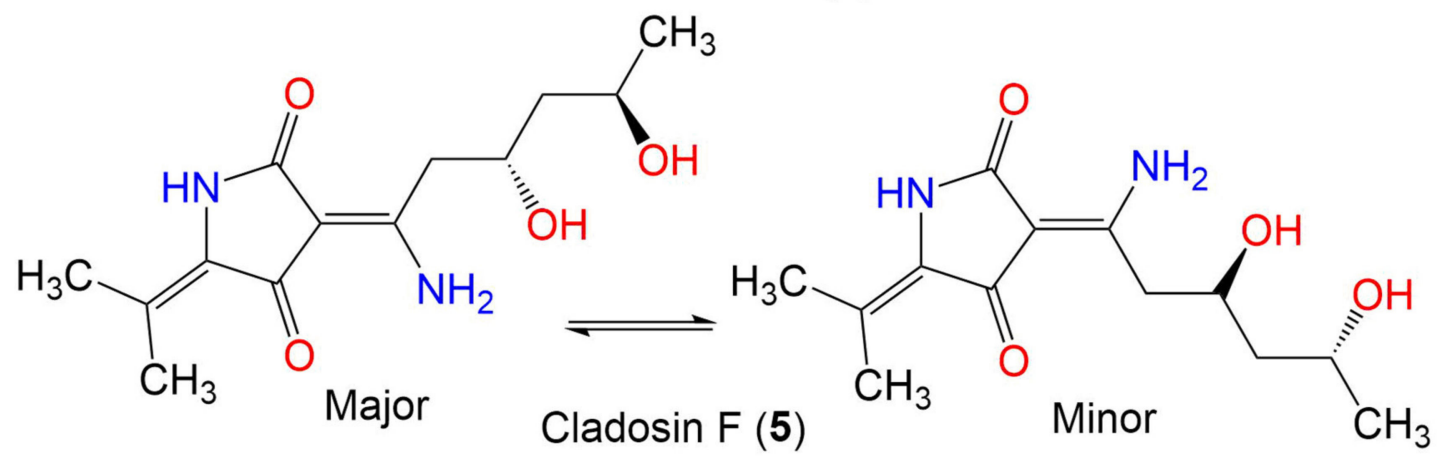

Figure 1. Tetramic acid derivatives 1-5. 
<smiles>CO[C@H](C/C(N)=C1\C(=O)NC(=C(C)C)C1=O)C[C@@H](C)C[C@H](C/C(N)=C1\C(=O)NC(=C(C)C)C1=O)OC</smiles><smiles>CO[C@@H](C[C@@H](C)O)C[C@@H](CC(Nc1ccccc1)=C1C(=O)NC(=C(C)C)C1=O)OC</smiles><smiles>CO[C@@H](C/C(=C1/C(=O)NC(=C(C)C)C1=O)[C@@H](C/C(Nc1ccccc1)=C1\C(=O)NC(=C(C)C)C1=O)OC)C[C@@H](C)O</smiles><smiles>CC(C)=C1NC(=O)C(=C(C[C@@H](C[C@H](C)O)Nc2ccccc2)Nc2ccccc2)C1=O</smiles>

Figure 2. Tetramic acid derivatives 6-9. 
<smiles>CC(C)=C1NC(=O)C(=C(C[C@@H](C[C@H](C)O)Nc2ccccc2)Nc2ccccc2)C1=O</smiles><smiles>CC(C)[C@H]1NC(=O)/C(=C(/N)C[C@@H](O)C[C@@H](C)O)C1=O</smiles>

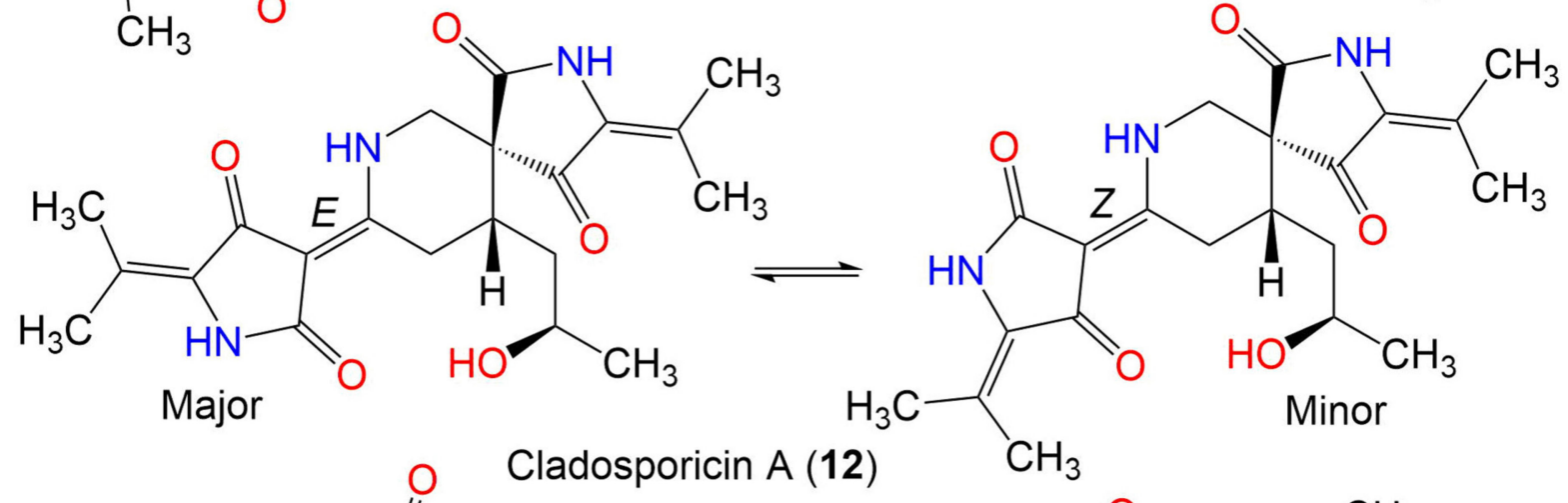

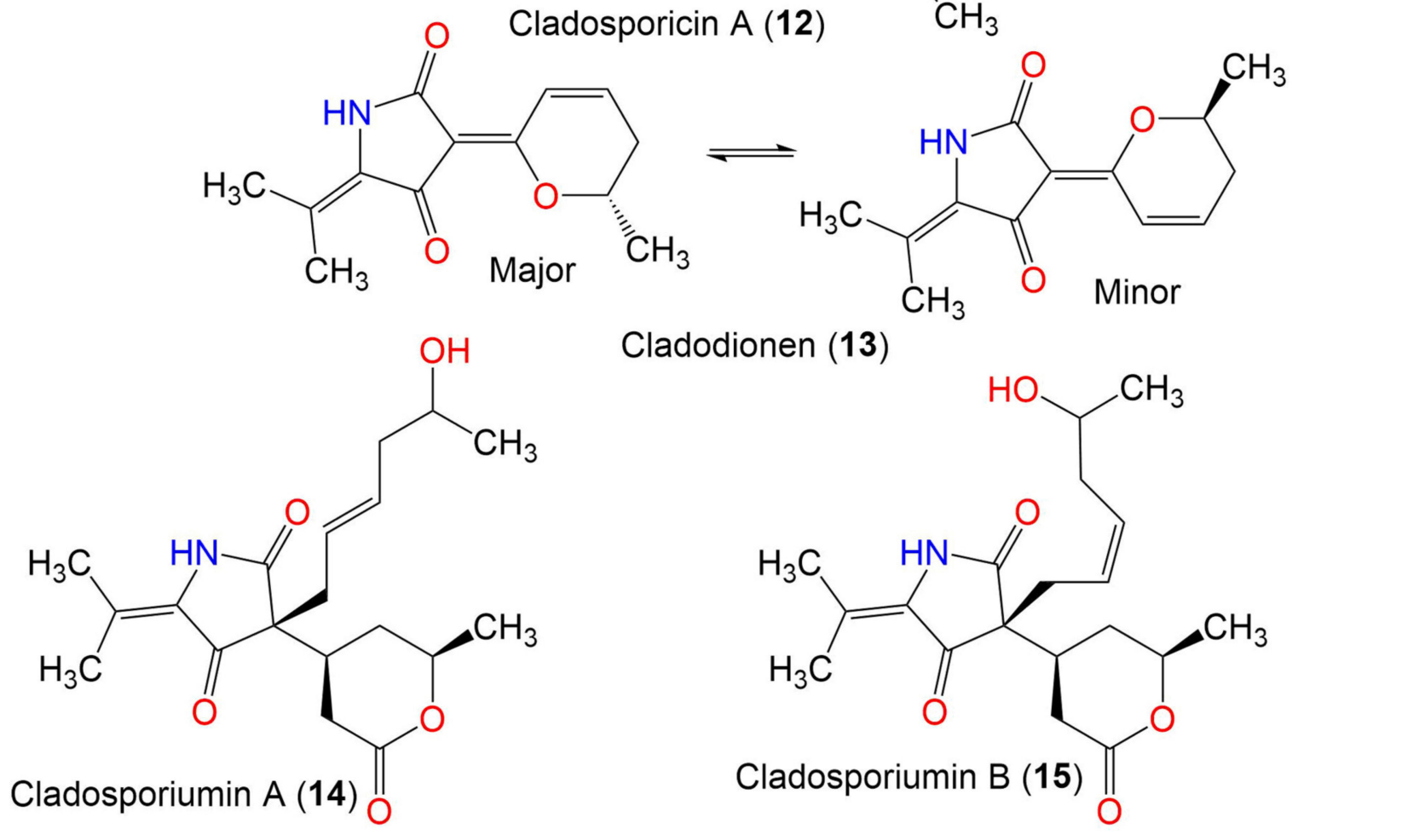

Figure 3. Tetramic acid derivatives 10-15. 
C. sphaerospermum EIODSF 008 isolated from the deep-sea sediment collected from the East Indian Ocean yielded tetramic acid derivatives 13 and 22-28 (Figures 4 and 5). They were assessed for cytotoxicity towards HL-60, HepG2, and MCF-7. Only $\mathbf{1 3}$ had cytotoxicity $\left(\mathrm{IC}_{50} 28.6 \mu \mathrm{M}\right)$ towards the HL-60 cell line [57]. Additionally, they showed no antibacterial potential towards E. coli, M. luteus, and B subtilis [57]. Additionally, $\mathbf{1 3}$ showed cytotoxic capacity towards HL-60, HeLa, HCT-116, and MCF-7 cell lines (IC I0 $_{50}$ ranged from 9.1 to $19.1 \mu \mathrm{M})$, compared to ADR (adriamycin) $\left(\mathrm{IC}_{50}\right.$ ranged from 0.02 to $\left.0.67 \mu \mathrm{M}\right)$. However, it did not have antibacterial activities (conc. $100 \mu \mathrm{g} / \mathrm{mL}$ ) against B. subtilis, P. aeruginosa, C. perfringens, S. aureus, E. coli, and C. albicans [56]. Compounds 14-21, new tetramic acid derivatives, were purified from the sea-sediment derived Cladosporium sp. acetone extract by Huang et al. in 2018. Compounds 14-16 are unusual 3-acyltetramic acids, having at C-3 of the pyrrolidine-2,4-dione core, a six-membered lactone ring, and hexyl-enic alcohol chain. They showed no obvious AchEI activity in the modified Ellman's enzyme assay [58]. Moreover, they displayed no anti-biofilm effect against $C$. albicans and S. aureus in the broth micro-dilution method and no cytotoxic effect towards HL60, HepG-2, and MCF-7 cell lines in the CCK8 assay [58].

\subsection{Diketopiperazines}

Diketopiperazines (DKPs) are cyclic dipeptides, consisting of two amino acids with or without extra structural modifications in the DKPs nucleus [108]. Their main skeleton comprises a six-membered piperazine nucleus produced from the double condensations among two amino acids $[129,130]$. The formation of peptide bonds in DKPs are catalyzed mainly by cyclodipeptide synthases (CDPSs) and non-ribosomal peptide synthetases (NRPSs) [131]. They possessed interesting bioactivities such as anti-Alzheimer, antimicrobial, antiviral, microtubule polymerization inhibitory, antitumor, anti-quorum-sensing, and haemosuppressor $[129,130,132]$.

Cyclo-(Val-Pro) (32) and cyclo-(Phe-Pro) (33) were separated from the EtOAc extract of Cladosporium sp. F14 isolated from seawater and investigated for their anti-larval activity at conc. $50 \mu \mathrm{g} / \mathrm{mL}$ towards Bugula neritina and Balanus amphitrite larvae in the settlement inhibition assays [60] (Figure 6). They inhibited B. neritina settlement $\left(\mathrm{EC}_{50} 70.43\right.$ and $>200 \mu \mathrm{g} / \mathrm{mL}$, respectively) and B. amphitrite settlement $\left(\mathrm{EC}_{50} 68.57\right.$ and $37.82 \mu \mathrm{g} / \mathrm{mL}$, respectively). Furthermore, 32 and 33 obviously prohibited L. hongkongensis growth (IZDs $8 \mathrm{~mm}$ and MICs 200 and $200 \mu \mathrm{g} / \mathrm{mL}$, respectively), compared to streptomycin (MIC $250 \mu \mathrm{g} / \mathrm{mL}$ ). The MICs of 33 towards Ruegeria sp. and M. luteus were 200 and $100 \mu \mathrm{g} / \mathrm{mL}$, respectively, compared to streptomycin (MIC 500 and $250 \mu \mathrm{g} / \mathrm{mL}$, respectively) [60]. On the other hand, thio-diketopiperazine derivatives, cladosporins A (36) and B (37), and haematocin (38) purified from the sediment-derived Cladosporium sp. were moderately cytotoxic towards HepG2 cell line ( $\mathrm{IC}_{50} 48,21$, and $42 \mu \mathrm{g} / \mathrm{mL}$, respectively) [62].

\subsection{Alkaloids}

Fungal alkaloids are nitrogen-containing metabolites that are derived from amino acid metabolism and the mevalonate pathway [133]. Many studies reported the detection of various classeses of alkaloids from marine-derived fungi such as pyrrolidine, indole, pyrrolizidine, quinazoline, quinoline, and purine classes [134-136]. These metabolites have shown broad biological activities: cytotoxic, anti-inflammatory, antioxidant, antibacterial, antifungal, antiviral, protease inhibitory. Therefore, they could have a potential for the development of innovative therapies [134-136]. In the current work, 49 alkaloids, belonging to different classes have been reported. Among them, 27 alkaloids were reported from unidentified Cladosporium species. 


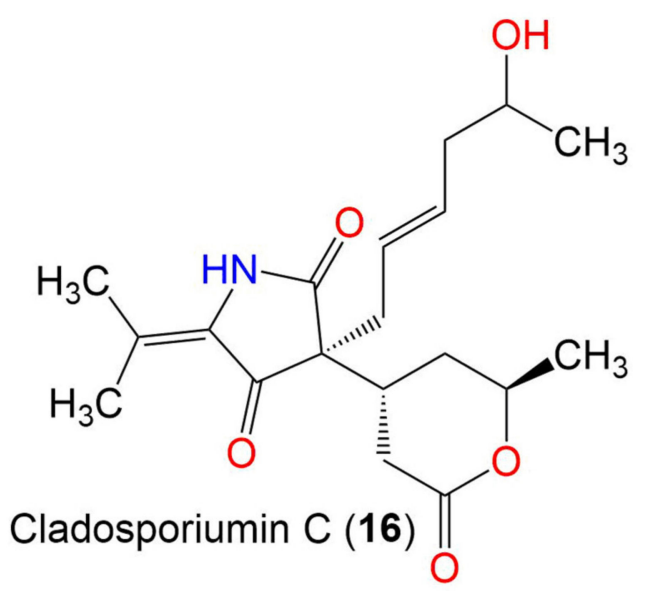<smiles>CC(C)=C1NC(=O)[C@](O)(/C=C/CCC(C)O)C1=O</smiles><smiles>CC(C)=C1NC(=O)C(=C(O)C=CC[C@@H](C)O)C1=O</smiles><smiles>CC(O)C/C=C/C(O)=C1/C(=O)N[C@@H](C(C)C)C1=O</smiles><smiles>CC(C)=C1NC(=O)C(=C(O)CC(O)CC(C)O)C1=O</smiles><smiles>CO[C@H](CC(O)=C1C(=O)N[C@@H](C(C)C)C1=O)CC(C)O</smiles>

Cladosporiumin Ha: anti C-11, C-13 (21) Cladosporiumin $\mathrm{Hb}$ : syn $\mathrm{C}-11, \mathrm{C}-13$<smiles>CC(C)[C@H]1NC(=O)/C(=C2/C=CC[C@H](C)O2)C1=O</smiles><smiles>[Z]C1=C(C(C)C)[C@@H](C(C)C)NC1=O</smiles><smiles>CC(C)=C1NC(=O)C2=C1O[C@H](C[C@@H](C)O)CC2=O</smiles>

Cladosporiumin $\mathrm{J}(\mathbf{2 3})$<smiles>CC(C)=C1NC(=O)C2=C1O[C@H](CC(C)O)CC2=O</smiles>

Cladosporiumin K (24)

Figure 4. Tetramic acid derivatives 16-24. 


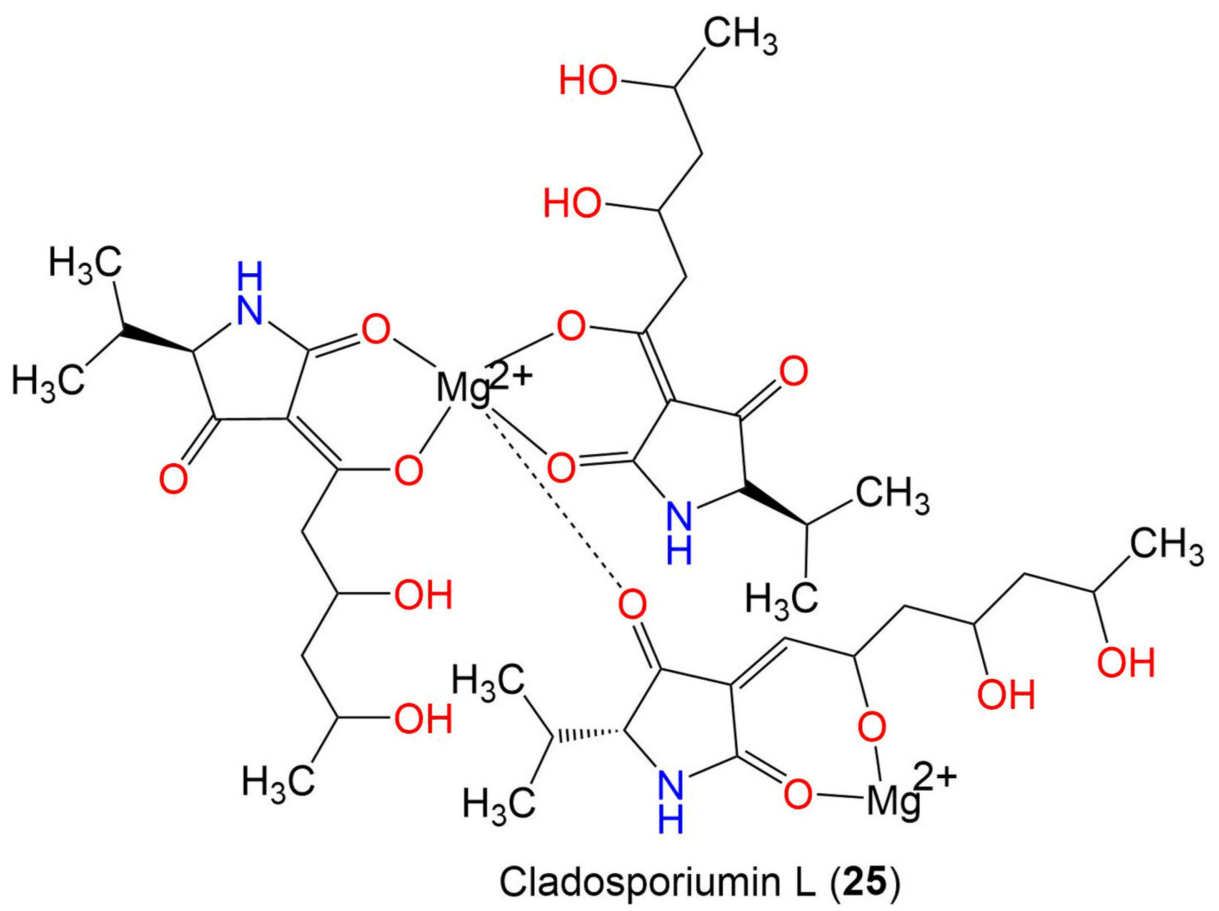<smiles>C/C=C/C=C/C(O)=C1/C(=O)NC(=C(C)C)C1=O</smiles><smiles>CC(C)[C@H]1NC(=O)/C(=C(O)\C=C\C[C@H](C)O)C1=O</smiles><smiles>CC(C)=C1NC(=O)C(=C(O)C=CC[C@@H](C)O)C1=O</smiles><smiles>CC(C)=C1NC(=O)[C@H]([C@]2(C/C=C/C[C@H](C)O)CC(=O)O[C@H](C)C2)C1=O</smiles>

Cladosporiumin I (29)<smiles>CC(C)=C1NC(=O)[C@](C/C=C/C[C@H](C)O)([C@H]2CC(=O)O[C@H](C)C2)C1=O</smiles>

Figure 5. Tetramic acid derivatives 25-30. 
<smiles>O=C1N[C@H](Cc2c[nH]c3ccccc23)C(=O)N2CCC[C@H]12</smiles><smiles>CC(C)[C@H]1NC(=O)[C@@H]2CCCN2C1=O</smiles><smiles>O=C1N[C@H](Cc2ccccc2)C(=O)N2CCC[C@H]12</smiles><smiles>CC(C)[C@H]1NC(=O)[C@H](Cc2ccccc2)NC1=O</smiles><smiles>CC(C)CC1NC(=O)CNC1=O</smiles>

Cyclo-(Gly-Leu) (35)<smiles>CC(=O)O[C@H]1C=CC=C2C[C@@]3(C)C(=O)N4[C@@H]5C(=CC=C[C@H]5O)C[C@]4(C)C(=O)N3[C@H]2[C@H]1C(C)(C)C</smiles><smiles></smiles>

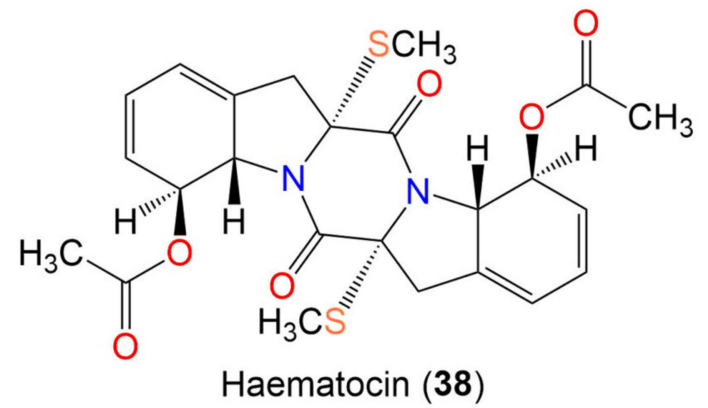

Figure 6. Diketopiperazine derivatives 31-38.

The glyantrypine-type alkaloids, 42-55, were separated from Cladosporium sp. PJX-41 isolated from mangrove and assessed for anti-H1N1 activity using CPE (cytopathic effect) inhibition assay (Figures 7 and 8). Compounds 45, 49, 51-53, and 55 displayed remarkable anti-H1N1 activities $\left(\mathrm{IC}_{50}\right.$ values ranged from 82 to $89 \mu \mathrm{M}$ ), compared to ribavirin $\left(\mathrm{IC}_{50}\right.$

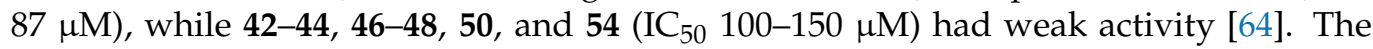
mycelium extract of the marine-derived Cladosporium sp. associated with Chondria crassicualis red alga afforded 56 that exhibited antioxidant potential $\left(\mathrm{ED}_{50} 82.0 \mu \mathrm{M}\right)$ more than oxybenzone (sunscreen agent, $\mathrm{ED}_{50} 350 \mu \mathrm{M}$ ) as evident by their UV-A protecting potential [65]. Furthermore, it had a moderate antibacterial effect towards multidrug-resistant and methicillin-resistant S. aureus and S. aureus with MICs 31.0, 62.5, and 62.5, $\mu \mathrm{g} / \mathrm{mL}$, respectively [65]. The quinolactacins and citrinadins alkaloids 58, 68, and 70 separated from C. oxysporum were assessed for anti-plasmodial potential towards chloroquine-sensitive Plasmodium falciparum 3D7 [66] (Figure 9). Only 58 (conc. $3.13 \mu \mathrm{g}$ to $25.0 \mu \mathrm{g}$ ) had an antiplasmodial effect $\left(\mathrm{EC}_{50} 24.8 \mu \mathrm{M}\right)$, while 68 and 70 displayed no activity $\left(\mathrm{EC}_{50}>25.0 \mu \mathrm{M}\right)$, compared to artesunate $\left(\mathrm{EC}_{50} 0.074 \mu \mathrm{M}\right)$ in the SYBR Green I assay. Further, 58 (conc. ranged from $6.25 \mu \mathrm{M}$ to $50.0 \mu \mathrm{M}$ for $24 \mathrm{~h}$ ) was investigated for apoptotic effect on 3D7plasmodia strain by measuring the parasite $\Delta \Psi \mathrm{m}$ (mitochondrial membrane potential). It induced loss of $\Delta \Psi \mathrm{m}$, leading to the release of cytochrome $\mathrm{C}$ from mitochondria to the cytosol resulted in parasite apoptosis. Therefore, it may provide a scaffold to apoptotic death in the stages of P. falciparum development [66]. Moreover, 58, 68, and $\mathbf{7 0}$ had no antiburuli ulcer activity against Mycobacterium ulcerans $\left(\mathrm{IC}_{50}>10 \mu \mathrm{M}\right.$ ), compared to rifampicin $\left(\mathrm{IC}_{50}<1 \mu \mathrm{M}\right)$ in the Resazurin microtiter assay [66]. 


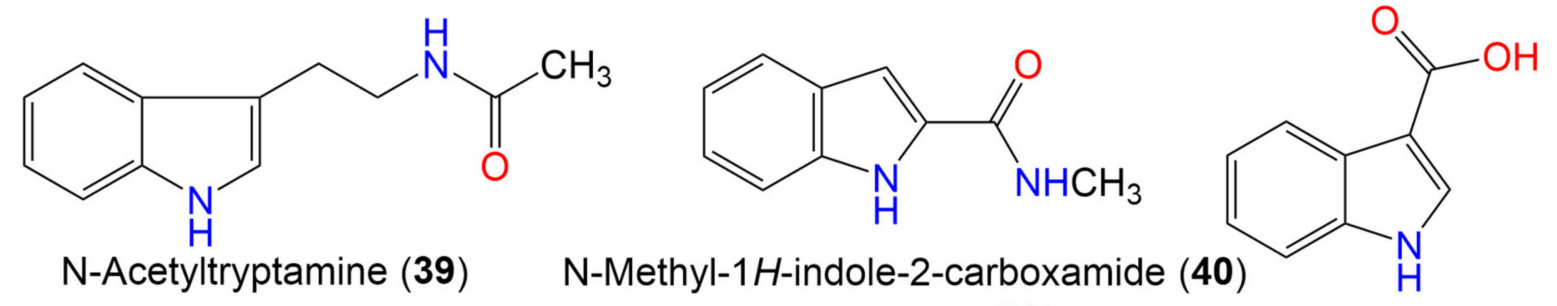<smiles>N#CCC[C@H]1C(=O)NCc2nc3ccccc3c(=O)n21</smiles><smiles>CCC[C@H]1C(=O)N[C@@H](O)c2nc3ccccc3c(=O)n21</smiles>

Indole-3-carboxylic acid (41)<smiles>Cc1c[nH]c2ccccc12</smiles><smiles></smiles>

14R-Oxoglyantrypine (44)<smiles>O=C1NC(=O)[C@H](Cc2c[nH]c3ccccc23)n2c1nc1ccccc1c2=O</smiles>

14S-Oxoglyantrypine (45)<smiles>O=C1N[C@@]2(CC3c4ccccc4N[C@H]32)c2nc3ccccc3c(=O)n21</smiles>

Prelapatin B (46)<smiles>C/C=C/C=C\C(=O)[C@@]1(O)C(=O)Nc2ccccc21</smiles>

Cladoquinazoline (47)<smiles>CC(C)[C@@H]1NC(=O)[C@H](C[C@]2(O)C(=O)Nc3ccccc32)n2c1nc1ccccc1c2=O</smiles>

Epi-Cladoquinazoline (48)<smiles>CC(C)=C1NC(=O)[C@@H](C[C@]2(O)c3ccccc3N3C(=O)[C@H](C)NC32)n2c1nc1ccccc1c2=O</smiles>

Norquinadoline A (49)

Figure 7. Alkaloids 39-49. 
<smiles>CC(C)=C1NC(=O)[C@H](C[C@]2(O)c3ccccc3N3C(=O)C(C)(C)NC32)n2c1nc1ccccc1c2=O</smiles>

Quinadoline A (50)<smiles>CC(=O)O[C@H](c1nc2ccccc2c(=O)n1[C@@H]1C[C@@]2(OC1=O)c1ccccc1N1C(=O)C(C)(C)N(O)[C@H]12)C(C)C</smiles>

Tryptoquivaline (53)<smiles>CC(=O)OC(c1nc2ccccc2c(=O)n1[C@@H]1C[C@@]2(OC1=O)c1ccccc1N1C(=O)[C@@H](C)N[C@H]12)C(C)C</smiles>

Deoxynortryptoquivaline (51)<smiles>CC(=O)O[C@H](c1nc2ccccc2c(=O)n1[C@@H]1C[C@@]2(OC1=O)c1ccccc1N1C(=O)C(C)(C)N[C@H]12)C(C)C</smiles>

Deoxytryptoquivaline (52)<smiles>CC(=O)OC(c1nc2ccccc2c(=O)n1[C@@H]1C[C@@]2(OC1=O)c1ccccc1N1C(=O)C(C)(C)N(O)[C@H]12)C(C)C</smiles>

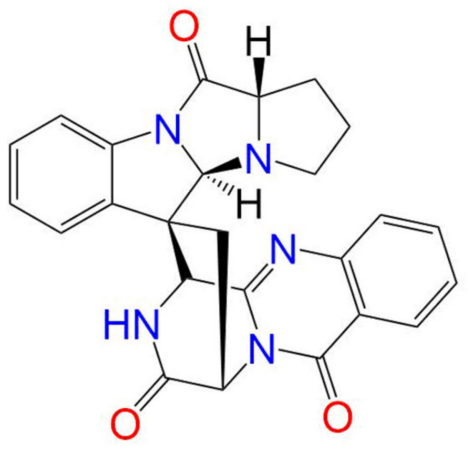

Quinadoline B (55)<smiles>CCC(C)[C@H]1NC(=O)c2c1n(C)c1ccccc1c2=O</smiles>

Circumdatin A (56)

Quinolactacin A1 (57)

Quinolactacin A2 (58)<smiles>CC(C)[C@H]1NC(=O)c2c1n(C)c1ccccc1c2=O</smiles>

Quinolactacin B1 (59)<smiles>CC(C)[C@H]1NC(=O)c2c1n(C)c1ccccc1c2=O</smiles>

Quinolactacin B2 (60)<smiles>CCC(C)[C@]1(O)NC(=O)c2c1n(C)c1ccccc1c2=O</smiles>

Quinolactacin C1 (61)

Figure 8. Alkaloids 50-61. 
<smiles>CC[C@H](C)[C@]1(O)NC(=O)c2c1n(C)c1ccccc1c2=O</smiles>

Quinolactacin C2 (62)<smiles>CC(C)C[C@@]1(O)NC(=O)c2c1n(C)c1ccccc1c2=O</smiles>

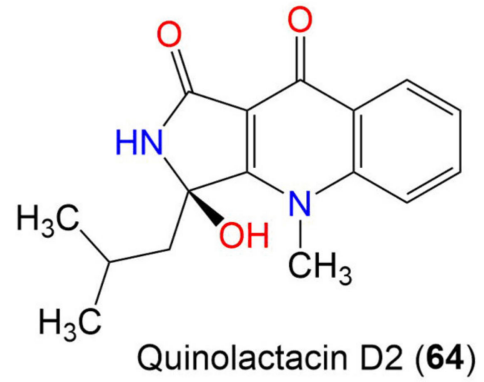<smiles>CC[C@H](C)[C@H]1NC(=O)c2cc3ccc(O)cc3[n+](C)c21</smiles>

Quinolactacin D1 (63)<smiles>O=C1c2c([nH]c3ccccc3c2=O)-c2cccn21</smiles><smiles>CC[C@@H]1CCC2=[N+](C)CCC2C1</smiles>

$\mathrm{CH}_{3}$ Quinocitrinine B (66) Quinolactacide (67)

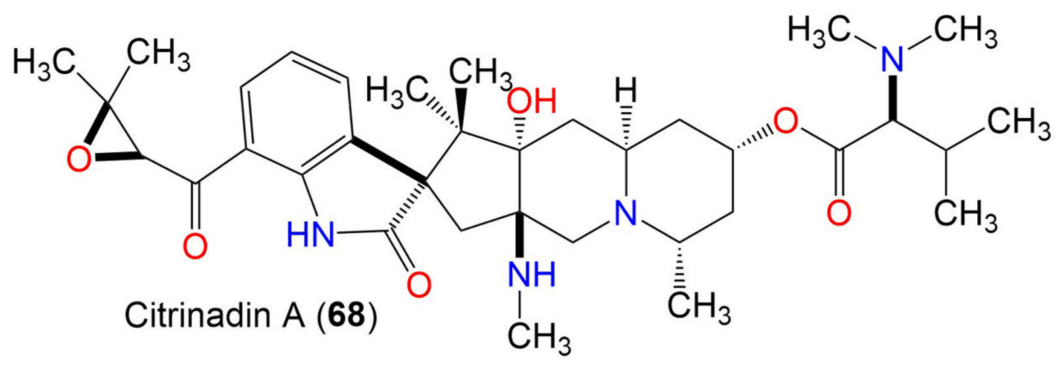<smiles></smiles><smiles>CN[C@@]12C[NH+]3[C@@H](C)C[C@H](OC(=O)C(C(C)C)N(C)C)C[C@H]3C[C@]1(C)[C@@](C)(OCC(C)=O)[C@@]1(C2)C(=O)Nc2c(C(=O)O)cccc21</smiles>

Figure 9. Alkaloids 62-70.

They had significant activity towards HepG-2 and MCF-7 ( $\mathrm{IC}_{50}$ ranging from 78.57 to $96.54 \mu \mathrm{M}$ and from 51.32 to $94.49 \mu \mathrm{M}$, respectively), compared to curcumin ( IC $_{50} 61.38$ and $20.68 \mu \mathrm{M}$, respectively). However, they showed moderate activity versus LNCap and LNCap ( $\mathrm{IC}_{50}$ ranging from 32.94 to $45.71 \mu \mathrm{M}$ and from 54.47 to $60.31 \mu \mathrm{M}$, respectively), in comparison to curcumin ( $\mathrm{IC}_{50} 6.15$ and $13.78 \mu \mathrm{M}$, respectively) in the MTT assay [66]. Cladosporium sp. HNWSW-1 associated with the mangrove plant Ceriops tagal biosynthesized compounds 74-76 that were assessed for their cytotoxic and $\alpha$-glycosidase inhibitory effects (Figure 10). Compound 75 had cytotoxicity versus SGC-7901, K562, and BEL-7042 
cell lines ( $\mathrm{IC}_{50} 41.7,25.6$, and $29.4 \mu \mathrm{M}$, respectively), whereas 76 revealed cytotoxic potential towards BEL-7042 and Hela cell lines ( $\mathrm{IC}_{50} 26.7$ and $14.9 \mu \mathrm{M} \mu \mathrm{M}$, respectively) in the MTT assay.<smiles>[H][R17](=O)OC(=O)CCC</smiles><smiles>[R16][R7]#P</smiles><smiles>CN[C@@]12CN3[C@H](C)C[C@@H](OC(C)=O)C[C@H]3C[C@@H]1[C@@H](C=O)[C@]1(C2)C(=O)Nc2c(C(=O)[C@@H]3OC3(C)C)cccc21</smiles>

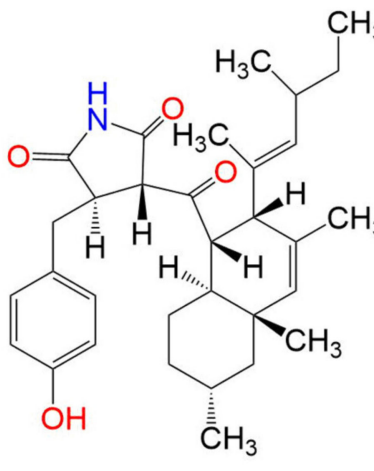

Cladosporitin A (74)<smiles>CC(C)=C1NC(=O)c2c1oc(=O)c1ccc(O)c(O)c21</smiles><smiles>C/C1=C/C(=O)N2CCC[C@H]2OCC1</smiles><smiles>CC(=O)N[C@@H](CCCNC(=O)/C=C(/C)CCOC(=O)C(C)C)C(C)=O</smiles>

Cladosporilactam A (78)

Figure 10. Alkaloids 71-79.

Additionally, 76 exhibited $\alpha$-glucosidase inhibitory activity ( $\left.\mathrm{IC}_{50} 78.2 \mu \mathrm{M}\right)$, compared to acarbose $\left(\mathrm{IC}_{50} 275.7 \mu \mathrm{M}\right)$ in the glucose oxidase method [67]. Cladosporamide A (77) separated from Cladosporium sp. TPU1507 derived from marine sponge was assessed for its inhibitory effect towards PTP1B (protein tyrosine phosphatase) and TCPTP (T-cell PTP), using an enzyme-based assay [68]. It had mostly equivalent inhibition towards TCPTP and PTP1B (IC 5048 and $54 \mu \mathrm{M}$, respectively), in comparison to oleanolic acid (IC 50 $0.9 \mu \mathrm{M}$ ) [68]. Cao et al. purified a new 7-oxabicyclic[6.3.0]lactam, 78, from a gorgonian- 
derived Cladosporium sp. collected from the South China Sea. It $\left(\mathrm{IC}_{50}\right.$ 0.76-3.11 $\left.\mu \mathrm{M}\right)$ exhibited significant cytotoxicity towards HeLa, P388, HT-29, and A549 cell lines [69]. On the other hand, it had weak antibacterial activity (MIC $>25.0 \mu \mathrm{M}$ ) in broth dilution assay towards B. cereus, T. halophilus, S. epidermidis, S. aureus, E. coli, P. putida, N. brasiliensis, and V. parahaemolyticus [69]. Cladosporium sp. SCNU-F0001 isolated from a mangrove plant yielded a novel lactam macrolide named cladospamide A (79) that was evaluated for cytotoxic effect (conc. $50 \mu \mathrm{M}$ ) versus MDA-MB-435, A549, HCT116, HepG2, and BT549 in the MTT method and for antimicrobial potential (conc. $100 \mu \mathrm{g} / \mathrm{mL}$ ) towards S. aureus, B. subtilis, E. coli, Salmonella ATCC 14028, and P. aeruginosa. Unfortunately, it exhibited no noticeable activity [70].

The new cyano-containing alkaloids, cladosporins A (80) and B (81) purified from Cladosporium sp. SCSIO z015 broth did not have an obvious anti-biofilm activity towards S. aureus, E. coli, and B. subtilis [36] (Figure 11).<smiles>Cc1c(O)cc(O)c2c1[C@H](C)[C@H](C)O[C@H]2C#N</smiles>

Cladosporin A (80)<smiles>Cc1c(O)cc(O)c2c1[C@H](C)[C@H](C)O[C@@H]2C#N</smiles>

Cladosporin B (81)<smiles>CC1=CC(C2CC(O)C(CO)O2)C(=O)NC1=O</smiles><smiles>O=C(O)c1cccnc1</smiles>

Nicotinic acid (83)<smiles>CC(=O)OCc1nc(C)c(C)nc1C</smiles>

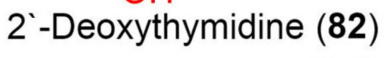

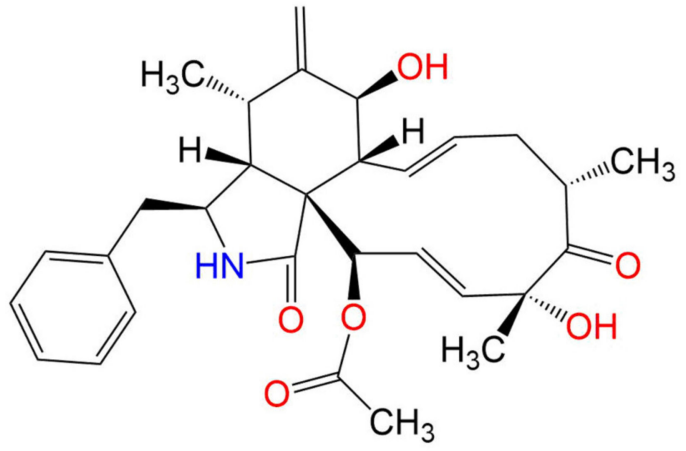

Cytochalasin D (85)<smiles>Cc1cccc(O)c1C(=O)N[C@H](C(=O)O)C(C)C</smiles><smiles>CC(=O)NCCc1ccc(O)cc1</smiles>

Figure 11. Alkaloids 80-87.

In the DPPH assay, they also had no activity $\left(\mathrm{IC}_{50}>100 \mu \mathrm{M}\right)$, compared to ascorbic acid $\left(\mathrm{IC}_{50} 4.9 \mu \mathrm{M}\right)$. Besides, they showed moderate toxicity towards brine shrine naupalii ( $\mathrm{LC}_{50} \mathrm{~s} 72.0$ and $81.7 \mu \mathrm{M}$, respectively), compared with toosendanin ( $\mathrm{LC}_{50} 21.2 \mu \mathrm{M}$ ) in the brine shrimp lethality assay [36]. In 2019, Bai et al. purified 84 and 85 from Cladosporium sp. JS1-2 isolated from the mangrove Ceriops tagal collected in the South China Sea. Compound $\mathbf{8 4}$ moderately prohibited the growth of Helicoverpa armigera Hubner newly hatched larvae ( $\mathrm{IC}_{50} 100 \mu \mathrm{g} / \mathrm{mL}$ ), compared to azadirachtin $\left(\mathrm{IC}_{50} 25 \mu \mathrm{g} / \mathrm{mL}\right.$ ). Further, they showed moderate antibacterial potential versus S. aureus with MICs 12.5 and $25.0 \mu \mathrm{g} / \mathrm{mL}$, respectively, compared with ciprofloxacin (MIC $0.39 \mu \mathrm{g} / \mathrm{mL}$ ) [71]. 


\subsection{Macrolides}

The term "macrolides" was first used to describe the natural antibiotics that have 12-16-membered macrocyclic lactone ring, functionalized by double bonds, and carrying different aminosaccharide and saccharide components [137]. Among these macrolides are 14-membered lactones (erythromycin and clarithromycin), 15-membered macrolides (azithromycin and spiramycin), and the 16-membered (avermectin B1a) that are clinically used macrolide antibiotics [138]. Members of this group possess a wide range of bioactivities such as antibacterial, anti-inflammatory, antiviral, antimalarial, antimitotic, and anticancer activity. They have been reported from various marine organisms $[138,139]$. The new 12-membered macrolide, cladospolide D (91), together with 88 and 89 were separated from Cladosporium sp. FT-0012 was obtained from Pohnpei Island, Federated State of Micronesia, and assessed for antimicrobial activity using paper disks at conc. $10 \mu \mathrm{g} /$ disk (Figure 12). Compound 91 exhibited activity versus M. racemosus KF223, B. subtilis KB27, and P. oryzae KB110 (IZDs 11.5, 16.0, and $14.0 \mathrm{~mm}$, respectively), while 88 was active (IZD $14.0 \mathrm{~mm}$ and $\mathrm{IC}_{50} 17.0 \mu \mathrm{g} / \mathrm{mL}$ ) towards X. campestris pv. oryzae. Moreover, 91 prohibited P. oryzae and M. racemosus growth ( $\mathrm{IC}_{50} \mathrm{~s} 29.0$ and $0.15 \mu \mathrm{g} / \mathrm{mL}$, respectively) [72].

Cladosporium sp. F14 isolated from seawater yielded a nine-membered macrolide, 92 that had weak antibacterial potential towards M. smegmatis, E. coli, B. thuringiensis, S. aureus, and B. subtilis and weak cytotoxic potential toward A435, HeLa, K562, and A549 in the MTT method [76]. C. herbarum isolated from Callyspongia aerizusa sponge yielded cladospolide B (89) and pandangolides 2-4 (95-97) that showed no antimicrobial potential versus S. aureus ATCC 25923, B. subtilis 168, E. coli ATCC 25922, and C. albicans in the agar plate diffusion assay [74]. Moreover, the EtOAc extract of Cladosporium sp. IFB3lp-2 isolated from the mangrove forest of Hainan province of China yielded 88, 89, 93-96, 100, and 116 that had no significant activity against HCT-116, Coxsachievirus A16, A549, MD-MBA-231, HepG2, human enterovirus 71, A375, and SW1116 cell lines (conc. $20 \mu \mathrm{M}$ ) in the MTT assay [73] (Figure 13).

Additionally, Cladosporium sp. SCNU-F0001 isolated from a mangrove plant biosynthesized a new macrolide thiocladospolide $\mathrm{E}$ (105), along with 89 that were evaluated for cytotoxic effect (conc. $50 \mu \mathrm{M}$ ) versus MDA-MB-435, A549, HCT116, HepG2, and BT549 in the MTT method and for antimicrobial potential (conc. $100 \mu \mathrm{g} / \mathrm{mL}$ ) towards S. aureus, B. subtilis, E. coli, Salmonella ATCC 14028, and P. aeruginosa. Unfortunately, none of them exhibited noticeable activity [70]. Cao et al. purified 12-membered macrolides 89 and 117-120 from a gorgonian-derived Cladosporium sp. collected from the South China Sea. They showed no cytotoxicity towards HeLa, P388, HT-29, and A549 cell lines [69]. Furthermore, they were evaluated for antibacterial activity in broth dilution assay towards B. cereus, T. halophilus, S. epidermidis, S. aureus, E. coli, P. putida, N. brasiliensis, and V. parahaemolyticus. Compounds 117-119 exhibited antibacterial potential against all tested bacteria (MIC values ranging from 3.13 to $25.0 \mu \mathrm{M}$ ), however $\mathbf{8 9}$ and $\mathbf{1 2 0}$ had weak activity (MIC $>25.0 \mu \mathrm{M}$ ) [69]. The metabolites 93 and 115 separated from Cladosporium sp. F14 at conc. $50 \mu \mathrm{g} / \mathrm{mL}$ had no anti-larval activity towards both B. neritina and B. amphitrite larvae in the settlement inhibition assays [60]. In 2019, Zhang et al. separated the new polyketides 98 and 99 and a known analog 93 from the rice culture EtOAc extract of $C$. cladosporioides associated with Bruguiera gymnorrhiza. Their configuration was established using ECD, modified Mosher's, and X-ray diffraction methods, as well as optical rotations to be $5 \mathrm{R}, 11 \mathrm{R}$ for 98 ; $11 \mathrm{R}$ for 99 ; and $3 R, 5 S, 11 S$ for 93 . They had weak AChEI activity $\left(\mathrm{IC}_{50}>50 \mu \mathrm{M}\right)$, in comparison to tacrine in the modified Ellman's method [40]. C. cladosporioides MA-299 obtained from the mangrove plant B. gymnorrhiza yielded 12-membered thio-macrolides 96 and 101-104 that were assessed for antimicrobial potential against E. tarda QDIO-2 and E. ictarda QDIO-9 (aquatic pathogens) and C. glecosporioides QDAU-2, B. sorokiniana QDAU-5, P. piricola Nose QDAU-15, and F. oxysporum f. sp. cucumerinum QDAU-8 (plant pathogenic fungi) in the microtiter plates assay. All metabolites revealed activity against $C$. glecosporioides (MIC 1 or $2 \mu \mathrm{g} / \mathrm{mL}$ ), compared to amphotericin B (MIC $0.5 \mu \mathrm{g} / \mathrm{mL}$ ). Moreover, 101 and 104 showed noticeable activity (MIC $1.0 \mu \mathrm{g} / \mathrm{mL}$ ) towards with E. tarda and E. ictarda, respec- 
tively, compared to chloramphenicol (MIC $0.5 \mu \mathrm{g} / \mathrm{mL}$ ), while 102 and 104 exerted obvious effectiveness (MIC $1.0 \mu \mathrm{g} / \mathrm{mL}$ ) versus F. oxysporum f. sp. cucumerinum, compared to amphotericin B (MIC $0.5 \mu \mathrm{g} / \mathrm{mL}$ ). The data revealed that sulfur substituent may influence the macrolides' bioactivities [39]. The newly reported 12-membered macrolides having thioethers 107-112 and the related formerly reported 93 and 101 isolated from mangrovederived C. oxysporum HDN13-314 had no cytotoxic activity versus HCT-116, BEL-7402, HL-60, A549, L-02, HeLa, K562, MGC-803, MCF-7, PC-3, SH-SY5Y, and MDA-MB-231 $\left(\mathrm{IC}_{50}>50 \mu \mathrm{M}\right)[78]$ (Figure 14).

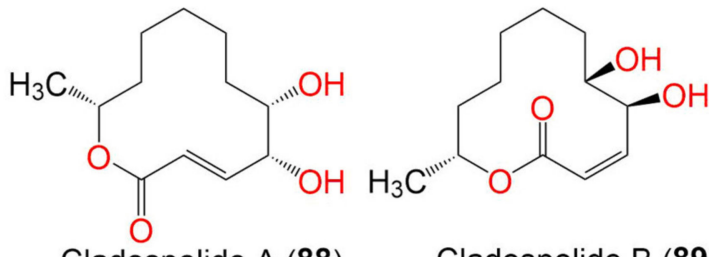

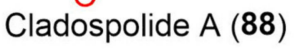
Cladospolide B (89)<smiles>C[C@@H]1CCCCC[C@@H](O)[C@@H](O)/C=C/C(=O)O1</smiles>

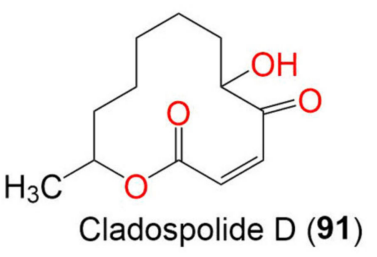<smiles>CC1OC(=O)C(O)CCCOC1=O</smiles>

Cladospolide C (90)<smiles>CC1CCCCCC[C@H](O)C(=O)[C@H](O)CC1=O</smiles>

Pandangolide 1a (94)

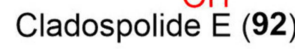<smiles>C[C@@H]1CCCCC[C@H](O)C(=O)[C@H](O)CC(=O)O1</smiles>

Pandangolide 1 (93)

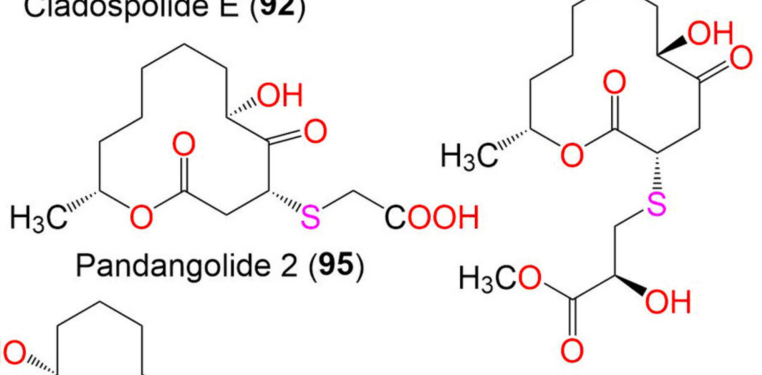

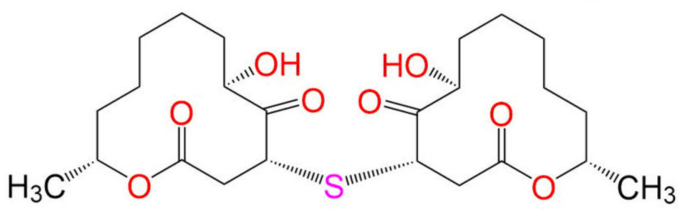

Pandangolide 4 (97)<smiles>C[C@H]1/C=C/CC[C@@H](O)CCCCC1=O</smiles><smiles>C[C@H]1/C=C/CC[C@@H](O)CCCCC(=O)O1</smiles>

$5 R$-Hydroxyrecifeiolide (98)

5S-Hydroxyrecifeiolide (99)

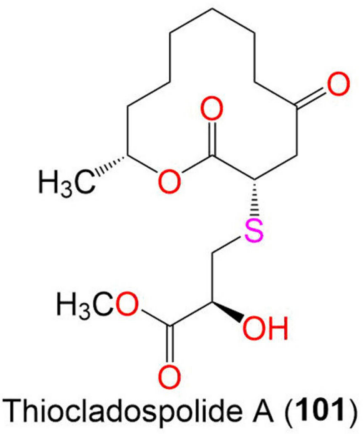<smiles>COC(=O)CS[C@H]1CC(=O)O[C@@H](C)CCCCCC[C@H]1O</smiles>

Methyl 2-(((4R,6R,12R)6-hydroxy-12-methyl-2,5dioxooxacyclododecan-4yl)thio)acetate (100)

Figure 12. Macrolides 88-101. 
<smiles>COC(=O)[C@H](O)CS[C@H]1CC(=O)C(=O)CCCCC[C@@H](C)OC1=O</smiles>

Thiocladospolide B (102)<smiles>C[C@H]1CCCCC[C@H](O)[C@@H](O)C[C@H](SCCO)C(=O)O1</smiles>

Thiocladospolide E (105)<smiles>COC(=O)[C@H](O)CS[C@H]1CC(=O)O[C@@H](C)CCCCCC[C@@H]1O</smiles>

Thiocladospolide G (108)

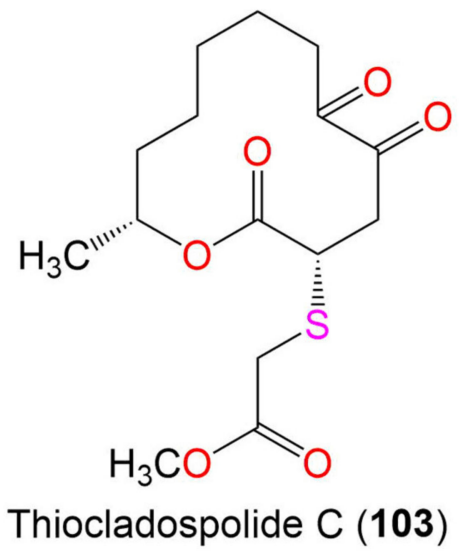<smiles>COC(=O)[C@H](O)CS[C@H]1CCCCCCC[C@@H](C)OC(=O)C1</smiles><smiles>C[C@H]1CCCCCC[C@H](O)C(=O)[C@H](S[C@@H]2C(=O)O[C@H](C)CCCCCC[C@H]2O)C(=O)O1</smiles>

Thiocladospolide F (107)

Thiocladospolide F (106)<smiles>C[C@H]1CCCCCC(O)C(=O)C[C@@H](SC[C@H](O)C(=O)O)C(=O)O[C@H](C)CCCCCCC(=O)C[C@@H]1SC[C@@H](O)C(=O)O</smiles>

Thiocladospolide G (109)<smiles>C[C@@H](CCCCCC[C@H](O)/C=C/C(=O)O)OC(=O)[C@@H](O)CS[C@@H]1CC(=O)[C@H](O)CCCCC[C@H](C)OC1=O</smiles>

Figure 13. Macrolides 102-111. 
<smiles>C[C@H]1CCCCC[C@@H](O)C(=O)C[C@H](SC[C@H](O)C(=O)O[C@@H](C)CCCCC[C@@H](O)[C@H]2C=CC(=O)O2)C(=O)O1</smiles>

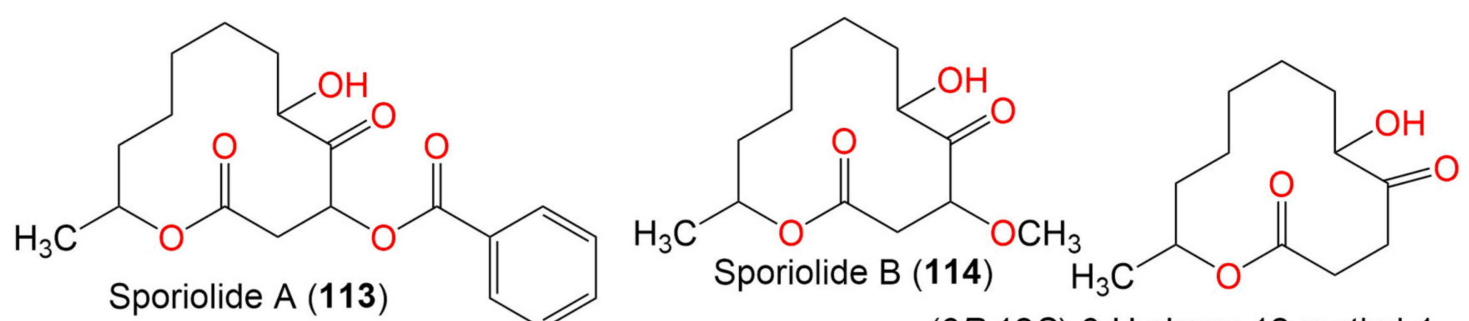

$(6 R, 12 S)-6$-Hydroxy-12-methyl-1-<smiles>CC(O)CCCCCCC(O)/C=C/C(=O)O[C@@H](CC(=O)[C@@H](O)CCCCCCC(C)O)C(=O)O[Mg]</smilesoxacyclododecane-2,5-dione (115) dioxooxacyclododecan-3-yl(E)-4,11 dihydroxydodec-2-enoate (116)<smiles>CO[C@H](CC(=O)C/C=C\[C@H](O)CC(=O)O)C[C@@H](C)OC</smiles>

Dendrodolide A (117)<smiles>C[C@H]1C[C@@H](O)CC(=O)C/C=C\[C@H](O)CC(=O)O1</smiles><smiles>C[C@H]1CCCC(=O)CCC[C@H](O)CC1=O</smiles>

$\mathrm{H}_{3} \mathrm{C}_{1 /}$<smiles>CO[C@@H](CC(=O)/C=C\CC(C)O)C[C@@H](O)CC(=O)O</smiles>

Dendrodolide C (118)

Dendrodolide L (119)

Dendrodolide M (120)<smiles>C[C@@H]1CCCCC2=C(O)C(=O)C[C@H]2C1C(=O)O[Mg]</smiles>

Figure 14. Macrolides 112-121.

Additionally, they exerted antibacterial activities versus the aquatic pathogens E. ictarda and E. tarda (MICs ranging from 4 to $32 \mu \mathrm{g} / \mathrm{mL}$ ), whereas 108 had the best effect (MIC 4 $\mu \mathrm{g} / \mathrm{mL}$ ) versus E. tarda [78]. In 2020, new thiomacrolides thiocladospolides $\mathrm{F}$ (106) and G (108) and cladocladosin A (121), a macrolide with bicyclo 5/9-ring, were purified from C. cladosporioides MA-299 by Zhang et al. and assessed for antimicrobial effect versus various plant, human, and aquatic pathogenic microbes in the microtiter plates assay. All metabolites revealed activity (MIC ranging from 1.0 to $4.0 \mu \mathrm{g} / \mathrm{mL}$ ) towards $V$. anguillarum and E. tarda (aquatic pathogenic bacteria) [79]. 
Moreover, 108 and 121 exerted activity (MICs $4.0 \mu \mathrm{g} / \mathrm{mL}$ ) towards H. maydis (plantpathogenic fungus) and $P$. aeruginosa (aquatic-pathogenic bacterium), respectively [79]. The new 12-membered macrolides, 113 and 114, purified from Cladosporium sp. L037 isolated from the Okinawan marine brown alga Actinotrichia fragilis exhibited cytotoxic influence ( $\mathrm{IC}_{50} 0.13$ and $0.81 \mu \mathrm{g} / \mathrm{mL}$, respectively) towards L1210 murine lymphoma cells in the MTT assay [80]. Moreover, $\mathbf{1 1 3}$ had antifungal potential against $C$. albicans, C. neoformans, A. niger, and N. crassa (MICs $8.4-16.7 \mu \mathrm{g} / \mathrm{mL}$ ), whereas 114 exhibited antibacterial activity only towards M. luteus and inactive against the other microorganisms [80].

\subsection{Butanolides and Butenolides}

Butanolides and butenolides are five-membered $\gamma$-lactones which may also be regarded as furan derivatives. They are an important class of structural motifs often encountered in various natural metabolites and synthetic targets [140]. They have an impressive range of bioactivities including antibiotic, antitumor, and anticancer that are intimately connected to their relative and absolute configurations [141].

The newly separated C12-macrolide, cladospolide F (122), purified from a soft coralassociated fungus Cladosporium sp. TZP-29, together with the formerly isolated derivative 126 showed no cytotoxic effect towards A-549, SMMC-7721, and HeLa cells in the SRB method [41]. Wuringege et al. reported that the butenolide, $\mathbf{1 2 6}$ isolated from Cladosporium sp. IFB3lp-2 exhibited no significant activity against HCT-116, Coxsachievirus A16, A549, MD-MBA-231, HepG2, human enterovirus 71, A375, and SW1116 cell lines (Conc. $20 \mu \mathrm{M}$ ) in the MTT assay [73]. Moreover, it showed no cytotoxicity towards various cancer cell lines: HeLa, P388, HT-29, HCT-116, BEL-7402, HL-60, A549, L-02, HeLa, K562, MGC803, MCF-7, PC-3, SH-SY5Y, MDA-MB-231, and A549 [69,78]. On the other hand, it had antibacterial activity in broth dilution assay towards B. cereus, T. halophilus, S. epidermidis, S. aureus, E. coli, P. putida, N. brasiliensis, and V. parahaemolyticus (MIC values ranging from 6.25 to $25.0 \mu \mathrm{M}$ ) [46]. Qi et al. stated that $\mathbf{1 2 6}$ displayed no anti-larval activity towards both B. neritina and B. amphitrite larvae in the settlement inhibition assays [60]. Additionally, it exerted antimicrobial activity versus E. ictarda and Cytospora mandshurica Miura (MIC $8 \mu \mathrm{g} / \mathrm{mL}$ ) [78]. The new metabolites 123, 124, and 127 and the known analog 126 separated from C. cladosporioides were assessed for AChEI activity using modified Ellman's method (Figure 15). Only 123 exhibited potent AChEI activity with the $\mathrm{IC}_{50}$ value of $40.26 \mu \mathrm{M}$, in comparison to tacrine, while other metabolites possessed weak activity $\left(\mathrm{IC}_{50}>50 \mu \mathrm{M}\right)$ [40].<smiles>C[C@H](O)CCCCCC[C@H]1OC(=O)C[C@H]1O</smiles><smiles>CCOC(=O)OCCCCCC[C@H]1OC(=O)C[C@H]1O</smiles><smiles>CCCCCCCCCCCCC[C@H](C)OC(C)=O</smiles><smiles>CCOC(=O)OCCCCCCC[C@H](C)O</smiles><smiles>C[C@H](O)CCCCC[C@H](O)[C@H]1C=CC(=O)O1</smiles><smiles>C[C@@H](O)CCCCC/C=C1\C=CC(=O)O1</smiles>

Iso-Cladospolide B (126)

Figure 15. Butenolides and butanolides 122-127. 


\subsection{Seco-Acids}

The seco-acids 128, 130, and $\mathbf{1 4 1}$ isolated from Cladosporium sp. IFB3lp-2 EtOAc extract had no noticeable cytotoxicity versus HCT-116, Coxsachievirus A16, A549, MDMBA-231, HepG2, human enterovirus 71, A375, and SW1116 cell lines (Conc. $20 \mu \mathrm{M}$ ) in the MTT assay [73]. Compound $\mathbf{1 3 1}$ did not show any anti-larval activity towards both B. neritina and B. amphitrite larvae [60]. Moreover, $\mathbf{1 3 2}$ did not have cytotoxic activity towards HCT-116, BEL-7402, HL-60, A549, L-02, HeLa, K562, MGC-803, MCF-7, PC-3, SH-SY5Y, and MDA-MB-231 ( $\left.\mathrm{IC}_{50}>50 \mu \mathrm{M}\right)$ [78], while it exhibited weak activity versus the aquatic pathogens E. ictarda, E. tarda, and Cytospora glecosporioides (MICs ranging from 16 to $32 \mu \mathrm{g} / \mathrm{mL}$ ) [78]. Cladospolide E (129) separated from a soft coral-associated Cladosporium sp. TZP-29, together with the formerly isolated derivatives $\mathbf{1 3 0}$ and $\mathbf{1 3 1}$ had no cytotoxic effect towards A-549, SMMC-7721, and HeLa cells in the SRB method. Moreover, 129-131 with $\mathrm{IC}_{50}$ ranged from 7.1 to $13.1 \mu \mathrm{M}$ remarkably reduced the accumulation of lipid elicited by oleic acid (OA) in the HepG2 liver cells, in comparison to lovastatin as determined by oilred $\mathrm{O}$ staining and intracellular triglyceride (TG) and total cholesterol (TC) quantification (Figure 16).

Further, they exhibited potent lipid-lowering potential in HepG2 hepatocytes, revealing a promising anti-hyperlipidemic capacity [41]. The new fatty acid esters 133, 134, and 138 and new fatty acids 135-137, 139, and 140 isolated from C. cladosporioides OUCMDZ187 obtained from the mangrove plant Rhizophora stylosa collected in Shankou, Guangxi Province of China showed no cytotoxic effects $\left(\mathrm{IC}_{50}>50 \mu \mathrm{M}\right)$ towards K562, A549, and HeLa cells in the SRB method [81]. Additionally, they revealed no antimicrobial activities (MIC $>150 \mu \mathrm{M}$ ) towards S. aureus CGMCC-1.2465, E. coli CGMCC-1.2389, E. aerogenes CGMCC-1.0876, P. aeruginosa CGMCC-1.1785, B. subtilis CGMCC-1.3376, and C. albicans CGMCC-2.2086 in the agar dilution method [81].

\subsection{Tetralones (Napthalenones)}

Tetralones comprise a bicyclic aromatic hydrocarbon and a ketone and are regarded as benzo-fused cyclohexanone derivatives. They played a substantial role as a starting material for the synthesis of a range of synthetic heterocyclic compounds and pharmaceuticals due to their potential reactivity and suitability [142]. Additionally, they are precursors of many natural metabolites and their derivatives. They have been used in the synthesis of therapeutically functional compounds such as antibiotics, acetylcholinesterase inhibitors, antidepressants, and antitumor alkaloids [142,143].

Cladosporone A (152), a new dimeric tetralone bridged via C-C linkage, was separated from Cladosporium sp. KcFL6 derived from the mangrove plant Kandelia candel, together with 142-144 (Figure 17). In anti-COX-2 assay, 144 and 152 displayed COX-2 inhibitory activities ( $\mathrm{IC}_{50} 60.2$ and $49.1 \mu \mathrm{M}$, respectively), in comparison to NS-398 and indomethacin [82]. Moreover, none of these metabolites had antimicrobial activities against A. baumannii ATCC-19606, S. aureus ATCC-29213, E. faecalis ATCC-29212, A. hydrophila ATCC-7966, E. coli ATCC-25922, K. pneumonia ATCC-13883, Fusarium sp., F. oxysporum f. sp. cucumeris, F. oxysporum f. sp. niveum, A. niger, and R. solani in the disc diffusion assay [82]. 
<smiles>CC(=O)/C=C/[C@@H](O)[C@@H](O)CCCCC[C@H](C)O</smiles>

Cladospolide A II (128)<smiles>CCCOC(=O)OC(Cl)=CCCC[C@H](C)O</smiles><smiles>C[C@@H](O)CCCCCCC(=O)/C=C/C(=O)O</smiles><smiles>C[C@@H](O)CCCCCC[C@H](O)/C=C/C(=O)O</smiles><smiles>[R]OC(=O)/C=C\C([R])C([R2])/C=C/CC([R3])CC([R3])O</smiles><smiles>COC(=O)CCCCCC/C=C/C(=O)OCC(O)CO</smiles><smiles>O=C(O)/C=C/C=C/CCCC(O)CO</smiles><smiles>CC(O)CCCCCC(=O)C(=O)CCC(=O)O</smiles>

11-Hydroxy-4,5-dioxododecanoic acid (141) 
<smiles>O=C1CC[C@H](c2ccc(O)c3c2C(=O)[C@H]2O[C@H]2[C@H]3O)c2cccc(O)c21</smiles>

Cladosporol (142)<smiles>O=C1CC[C@H](c2ccc(O)c3c2C(=O)CC[C@H]3O)c2cccc(O)c21</smiles>

Cladosporol C (143)<smiles>C[C@@H]1CC(=O)c2c([C@H]3CCC(=O)c4c(O)cccc43)ccc(O)c2[C@H]1O</smiles>

Cladosporol D (144)<smiles>CO[C@H]1CCC(=O)c2c([C@H]3CCC(=O)c4c(O)cccc43)ccc(O)c21</smiles>

Cladosporol F (146)<smiles>O=C1CC[C@H](c2ccc(O)c3c2C(=O)[C@H](O)[C@@H](O)[C@@H]3O)c2cccc(O)c21</smiles>

Cladosporol E (145)<smiles>CCCCCCC[C@H]1CCC(=O)c2c(O)cccc21</smiles><smiles>CO[C@H]1CCC(=O)c2c([C@H]3CCC(=O)c4c(O)cccc43)ccc(O)c21</smiles><smiles>O=C1CC[C@H](c2ccc(O)c3c2C(=O)CCC3=O)c2cccc(O)c21</smiles>

Cladosporol G (147) Cladosporol G (148)

Cladosporol H (149)<smiles>O=C1CC[C@H](O)[C@@H](c2ccc(O)c3c2[C@@H](O)CCC3=O)c2cccc(O)c21</smiles><smiles>O=C1CC[C@H](c2ccc(O)c3c2C(=O)CC[C@H]3O)c2cccc(O)c21</smiles>

Cladosporol I (150) Cladosporol J (151)<smiles>O=C1CC[C@H](c2ccc(O)c3c2C(=O)[C@H](O)[C@H]2O[C@H]32)c2cccc(O)c21</smiles>

Cladosporone A (152)<smiles>O=C1CCC(O)c2c([C@@H]3CC=Cc4c(O)cccc43)ccc(O)c21</smiles>

Altertoxin XII (153)

Figure 17. Tetralones (napthalenones) 142-153.

Compounds 143 and 152 had moderate cytotoxic activity towards Huh-7, K562, HL-60, MCF-7, H1975, U937, A549, BGC823, MOLT-4, and HeLa cell lines (IC 50 of 143 ranging from 11.4 to $72.5 \mu \mathrm{M}$ and for $\mathbf{1 5 2}$ ranging from 10.1 to $53.7 \mu \mathrm{M})$, compared to trichostatin $\mathrm{A}$ in the trypan blue-cell viability assay [82]. Zurlo et al. reported that $\mathbf{1 4 2}$ had a remarkable anti-proliferative potential towards SW480, HT-29, and CaCo-2, in particular towards HT-29. It was revealed that HT-29 cells exposure to 142 produced G1/S phase cell cycle arrest, assisted by a vigorous p21 waf1/cip1 expression, a significant down-regulation of CDK4, CDK2, cyclin E, and cyclin D1, and repression of CDK4 and CDK2 kinase activity [144]. It was demonstrated that its antiproliferative potential towards HT-29 cells was mediated via activation PPAR $\gamma$, resulting in upregulation of $\mathrm{p} 21^{\text {waf1/cip1 }}$ expression and inducing 
degradation of $\beta$-catenin, as well as impairing TCF/ $\beta$-catenin pathway as evident by reduced cyclin D1 and c-Myc transcription. Finally, it induced the expression of E-cadherin, therefore antagonizing invasion and metastasis [145]. C. cladosporioides HDN14-342 isolated from marine sediments yielded tetralone derivatives 143, 145-147, 154, and 155 that were evaluated for cytotoxic activities towards HCT-116, HeLa, and A549 cell lines by SRB method and towards HL-60 and K562 cell lines by MTT method, in comparison to doxorubicin ( $\left.\mathrm{IC}_{50} 0.2-0.8 \mu \mathrm{M}\right)$. Compounds 146 and 147 were active towards K562, HeLa, and HCT-116 cell lines ( $\mathrm{IC}_{50}$ ranging from 3.9 to $23.0 \mu \mathrm{M}$ ), while other metabolites had no activity $\left(\mathrm{IC}_{50}>50.0 \mu \mathrm{M}\right)$ [83]. In 2020, He et al. reported that 143 possessed no anti-allergic effect (IC50 $>200 \mu \mathrm{M}$ ) on RBL-2H3 cells, in comparison to loratadine (IC50 $35.01 \mu \mathrm{M}$ ) using fluorometric assay [75]. In 2017, Li et al. separated six cladosporol derivatives, cladosporol C (143) and cladosporols F-J (146 and 148-151) from the marine algal-derived C. cladosporioides EN-399 and evaluated their cytotoxic activities towards H446, A549, HeLa, L02, Huh7, LM3, SW1990, and MCF-7 using MTT assay. Note that 143, 148, and 149 displayed cytotoxic activities towards most of the tested cell lines with $\mathrm{IC}_{50}$ ranging from 1.0 to $20.0 \mu \mathrm{M}$. Notably, 149 had cytotoxic effect towards LM3, A549, and Huh7 cell lines ( $\mathrm{IC}_{50} 4.1,5.0$, and $1.0 \mu \mathrm{M}$, respectively), compared to cisplatin ( IC $_{50} 1.3 \mu \mathrm{M}$ for A549 and $9.1 \mu \mathrm{M}$ for LM3) and fluorouracil ( $\mathrm{IC}_{50} 6.2 \mu \mathrm{M}$ for Huh7), whereas 143 exhibited cytotoxic activity ( $\mathrm{IC}_{50} 4.0 \mu \mathrm{M}$ ) towards $\mathrm{H} 446$ cell line, compared to adriamycin $\left(\mathrm{IC}_{50} 4.0 \mu \mathrm{M}\right)$. These results revealed that the existence of dihydro-1,4-naphthoquinone nucleus was important for the activity (149 vs. 146, 148, and 143, 150, and 151) and C-4 methoxyl strengthened the activity (148 vs. 151) [84].

Moreover, their antimicrobial potential was assessed versus E. coli, A. hydrophila, S. aureus, E. tarda, P. aeruginosa, M. luteus, V. alginolyticus, V. parahemolyticus, V. harveyi, A. brassicae, F. oxysporum, G. graminis, C. gloeosporioides, and P. piricolav using micro-plate assay. Compounds 143,146 , and 148-151 showed inhibitory potential towards M. luteus, E. coli, and V. harveyi (MICs 4-128 $\mu \mathrm{g} / \mathrm{mL}$ ). None of them had activity (MIC $>128 \mu \mathrm{g} / \mathrm{mL}$ ) towards other tested microbes [84]. Bai et al. purified $\mathbf{1 4 3}$ and $\mathbf{1 4 5}$ from Cladosporium sp. JS1-2 isolated from the mangrove Ceriops tagal collected in the South China Sea [71]. Compound 145 prohibited the growth of Helicoverpa armigera Hubner newly hatched larvae $\left(\mathrm{IC}_{50} 150 \mu \mathrm{g} / \mathrm{mL}\right.$ ), compared to azadirachtin ( $\mathrm{IC}_{50} 25 \mu \mathrm{g} / \mathrm{mL}$ ) [71]. Further, they showed antibacterial potential versus S. aureus with MIC 6.25 and $1.56 \mu \mathrm{g} / \mathrm{mL}$, respectively, compared with ciprofloxacin (MIC $0.39 \mu \mathrm{g} / \mathrm{mL}$ ) [71]. Cladosporium sp. KFD33 isolated from blood cockle collected from Haikou Bay produced 150 and 153 that exhibited quorum sensing inhibitory potential towards Chromobacterium violaceum CV026 (MICs 30 and $20 \mu \mathrm{g} /$ well, respectively) in the well diffusion assay [85]. Nevertheless, 156 had no observable cytotoxic activity towards SF-268, NCI-H460, MCF-7, and HepG-2 (conc. $100 \mu \mathrm{M}$ ) in the SRB assay [87]. The new naphthalenone derivative 157, in addition to 156, 158, and 159 isolated Cladosporium sp. JJM22 associated with the mangrove plant $C$. tagal had no cytotoxic effect $\left(\mathrm{IC}_{50}>10 \mu \mathrm{M}\right)$ versus HeLa cell line in the MTT assay, compared to epirubicin [88] (Figure 18). In the micro-plate assay, only 158 exhibited noticeable antibacterial potential towards S. aureus, B. cereus, E. coli, V. alginolyticus, $V$. parahemolyticus, and MR S. aureus (conc. $20 \mu \mathrm{M}$ ) [88]. One new tetralone derivative, aladothalen (160) and previously reported (3S,4S)-3,4,8-trihydroxy-1-tetralone (159) were isolated from a sediment-associated Cladosporium sp. HDN17-58 (Figure 17). Note that 160 possessed potent bacteriostatic potential versus Mycobacterium phlei, B. cereus, and MRCNS (methicillin-resistant coagulase-negative Staphylococci) (MIC values of 25, 50, and $25 \mu \mathrm{M}$, respectively), compared to ciprofloxacin [89]. 
<smiles>CC(=O)C[C@]1(O)C(=O)c2c(O)ccc([C@@H]3CCC(=O)c4c(O)cccc43)c2C1=O</smiles>

Clindanone A (154)<smiles>CC(=O)C[C@]1(O)C(=O)c2c(O)ccc([C@H]3CCC(=O)c4c(O)cccc43)c2C1=O</smiles>

Clindanone B (155)<smiles>O=C1CCC(O)c2cccc(O)c21</smiles>

Isosclerone (156)<smiles>O=C1C[C@H](O)[C@H](O)c2cccc(O)c21</smiles><smiles>Cc1cc2c(c(O)c1C)C(=O)C[C@@H](O)[C@H]2O</smiles><smiles>Cc1cc2c(c(O)c1C)C(=O)C[C@H](O)C2</smiles>

(3R,4R)-3,4-Dihydro3,4,8-trihydroxy $-1(2 H)$-napthalenone

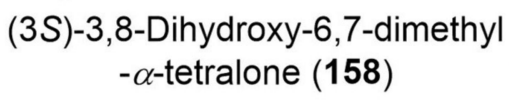
- $\alpha$-tetralone (158)

(-)-trans-(3R,4R)-3,4,8-Trihydroxy-

6,7-dimethyl-3,4-dihydro naphthalen-1(2H)-one (157)<smiles>O=C1c2c(O)cc(O)cc2CC[C@H]1O</smiles>

Figure 18. Tetralones (napthalenones) 154-160.

\subsection{Perylenequinones}

Perylenequinones comprise a class of natural products characterized by an oxidized pentacyclic core. They are dark-colored pigments isolated from diverse sources such as mold species, plants, and aphids [146]. They reported to have anthelmintic, photoactivity, antiviral and antitumor [146].

Four new perylenequinone derivatives, altertoxins VIII-XI (161-164), were isolated from Cladosporium sp. KFD33 (Figure 19). They exhibited quorum sensing inhibitory potential towards C. violaceum CV026 with MICs ranging from 20 to $30 \mu \mathrm{g} /$ well in the well diffusion assay [85]. Structurally, these metabolites related to altertoxins I-III previously were reported from Alternaria alternata [147].<smiles>O=C1CCc2c3c4c(cccc4c4ccc(O)c1c24)CC[C@H]3O</smiles>

Altertoxin VIII (161)<smiles>Oc1ccc2c3c1CCCc1cccc2c1c1c3[C@H](O)CC1</smiles>

Altertoxin IX (162)<smiles>Oc1ccc2c3c1CCC[C@@H](O)c1cccc2c1c1c3CCC1</smiles><smiles>CO[C@H]1CCc2c3c4c(c(O)ccc4c4cccc1c24)CCC3</smiles>

Altertoxin $X(163)$

Figure 19. Perylenequinone 161-164. 


\subsection{Naphthalene Derivatives}

Naphthalenes are a class of arenes containing two ortho-fused benzene rings that have been reported from plants, liverworts, fungi, and insects [148]. Their derivatives exhibited anti-inflammatory, antimicrobial, antioxidant, anti-protozoal, cytotoxic, and anti-platelet aggregation activities [148].

Cladosporium sp. associated with the mangrove $C$. tagal biosynthesized the naphthalene derivatives 166-168 that had anti-inflammatory potential via in-vitro inhibition of induced NO (nitric oxide) production by LPS (lipopolysaccharide) in RAW264.7 cells [91] (Figure 20). The mangrove-associated fungus Cladosporium sp. JJM22 yielded new naphthalene-chromane derivatives, cladonaphchroms A (169) and B (170), and related metabolites 165 and 168 that were assessed for antibacterial effectiveness versus S. albus ATCC-8799, E. coli ATCC25922, B. subtilis ATCC-6633, Micrococcus tetragenus ATCC-13623, and M. luteus ATCC-9341, employing microplate assay. Compound 169 possessed significant potential against $S$. albus (MIC $1.25 \mu \mathrm{g} / \mathrm{mL}$ ), compared to ciprofloxacin (MIC $0.6 \mu \mathrm{g} / \mathrm{mL}$ ). Moreover, 169 and 170 demonstrated broad-spectrum antifungal activities (MICs $25.0-100.0 \mu \mathrm{g} / \mathrm{mL}$ ) towards P. parasitica var. nicotianae, A. brassicicola, B. oryzae, C. capsici, C. paradoxa Moreau, and D. medusaea Nitschke, compared to pochloraz (MICs 12.5-50.0 $\mu \mathrm{g} / \mathrm{mL}$ ) [90]. Wu et al. stated that 166 had no cytotoxic effect $\left(\mathrm{IC}_{50}>10 \mu \mathrm{M}\right)$ versus HeLa cell line in the MTT assay and no antibacterial activity towards S. aureus, B. cereus, E. coli, V. alginolyticus, $V$. parahemolyticus, and MR S. aureus (conc. $20 \mu \mathrm{M}$ ) in the microplate assay [88].<smiles>COc1cccc2cccc(O)c12</smiles>

8-Methoxynaphthalen-1-ol (165)<smiles>COc1cccc2cccc(OC)c12</smiles>

1,8-Dimethoxynaphthalene
(166)<smiles>COc1ccc(O)c2cccc(O)c12</smiles>

4-Methoxynaphthalene-1,5-diol (167)<smiles>COc1c(O)ccc2cccc(O)c12</smiles>

8-Methoxynaphthalene1,7-diol (168)<smiles>COc1cccc2c([C@@H]3C[C@H](C)Oc4cccc(O)c43)ccc(OC)c12</smiles>

Cladonaphchrom A (169)<smiles>COc1cccc2c([C@@H]3C[C@H](C)Oc4cccc(O)c43)ccc(OC)c12</smiles>

Cladonaphchrom B (170)

Figure 20. Naphthalene derivatives 165-170.

\subsection{Xanthones}

Xanthones are secondary metabolites commonly reported from plants, fungi, and lichen [149]. They are heterocyclic metabolites with a xanthene-9-one framework, which is connected to different functional groups: methoxy, hydroxyl, prenyl, and dihydrofuran [150]. These metabolites showed diverse bioactivities: anti-HIV, anti-leishmanial, antitumor, anti-quorum sensing, antimicrobial, anti-inflammatory, antimalarial, advanced glycation end-products inhibitory, antioxidant, antihypertensive, and cytotoxic [150,151].

C. halotolerans GXIMD 02502 associated with the coral Porites lutea yielded compounds 171-177 that were evaluated for their cytotoxicity versus 22RV1 and C4-2B (prostatic cancer cell lines), as well as RWPE-1 (normal prostate epithelial cell). Among them, 171-173, 175, and $\mathbf{1 7 6}$ revealed notable cytotoxicity versus C4-2B and 22RV1 cells (inhibitions ranged 
from $55.8 \%$ to $82.1 \%$ at conc. $10 \mu \mathrm{M}$ ), whereas 176 was the potent one (inhibitions $77.7 \%$ and $82.1 \%$, respectively). On the other hand, they exhibited nearly no cytotoxic effect versus RWPE-1 cell (inhibition $<27 \%$ at conc. $10 \mu \mathrm{M}$ ) [92] (Figure 21).<smiles>Cc1cc(O)c2c(=O)c3c(C(=O)O)cccc3oc2c1</smiles>

8-Hydroxy-6-methylxanthone1-carboxylic acid (171)<smiles>COC(=O)c1cccc2oc3cc(CO)cc(O)c3c(=O)c12</smiles>

Methyl 8-hydroxy-6-(hydroxymethyl)9-oxo-9H-xanthene-1-carboxylate (173)<smiles>COC(=O)c1cccc2oc3cc(C)cc(O)c3c(=O)c12</smiles>

Methyl 8-hydroxy-6-methyl-9-oxo-9H -xanthene-1-carboxylate (172)<smiles>COC(=O)c1cccc2oc3cc(C(=O)O)cc(O)c3c(=O)c12</smiles>

8-(Methoxycarbonyl)-1-hydroxy-9-oxo-9Hxanthene-3-carboxylic acid (175)<smiles>COC(=O)c1cc(O)cc2oc3cc(CO)cc(O)c3c(=O)c12</smiles>

Figure 21. Xanthones 171-177.

\subsection{Tropolones}

Tropolones are natural metabolites with a cyclohepta-2,4,6-trienone moiety [152]. They are known to be produced by fungi, bacteria, and plants. It was reported to display diverse bioactivities, including antimicrobial, antiviral, anti-HIV, hepatitis, anti-inflammatory, and anticancer [152].

Silber et al. reported the isolation of malettinins A-C (178-180), along with the new metabolite, malettinin E (181) from Cladosporium sp. strain KF501 isolated from the German Wadden Sea (Figure 22). These metabolites have dihydropyran/tropolone structures connected to a furan ring. The configuration of 181 was determined by the single-crystal X-ray diffraction method. Interestingly, this was the first report for tropolones isolation from genus Cladosporium. They were evaluated for antimicrobial activity towards X. campestris, B. subtilis, S. epidermidis, C. albicans, and Trichophyton rubrum using the microplate assay. Note that 178-181 exhibited weak antifungal potential towards Trichophyton rubrum ( $\left.\mathrm{IC}_{50} 30.7-83.2 \mu \mathrm{M}\right)$, whereas 179-181 exhibited weak antibacterial effect towards Xanthomonas campestris ( $\left.\mathrm{IC}_{50} 28.3-37.9 \mu \mathrm{M}\right)$, compared to chloramphenicol $\left(\mathrm{IC}_{50} 2.1 \mu \mathrm{M}\right)$ [93]. 
<smiles>CC1=CO[C@]2(Oc3cc(O)c(=O)cc(C)c3C[C@H]2C)[C@@H]1C(=O)[C@H](C)C(C)(C)C</smiles>

Figure 22. Tropolones 178-181.

\subsection{Binaphthopyrones}

Bisnaphthopyrones are dimers, belonging to naphthopyrones. They have $\mathrm{C} 13$ basic skeleton (C6-C4-C3) that consists of naphthalene and pyrone cores [153].

The new binaphthopyrone, cladosporinone (182), and the formerly isolated viriditoxin (183) and viriditoxin derivatives (184 and 185) were separated the sediment associated C. cladosporioides (Figure 23). Note that 183 was firstly reported from Aspergillus viridinutans [154]. They were assayed for their cytotoxic potential versus L5178Y cells in the MTT assay. Compound 183 was the most potent one $\left(\mathrm{IC}_{50} 0.1 \mu \mathrm{M}\right)$, however $\mathbf{1 8 2}$ and $\mathbf{1 8 4}$ had a cytotoxic effect ( $\mathrm{IC}_{50} 0.88$ and $0.25 \mu \mathrm{M}$, respectively). However, 185 was ineffective [94]. Note that all metabolites had selective potential towards S. aureus ATCC-29213, with 183 being the most effective (MIC $0.023 \mu \mathrm{M}$ ) [94].

\subsection{Benzopyranes, Benzopyrones, and Pyrones}

Wang et al. reported the separation of compounds 188-190, 193, 200, 201, and 203 from Cladosporium sp. OUCMDZ-302 isolated from mangrove plant Excoecaria agallocha. They possessed no cytotoxic effect towards BEL-7402, A549, HeLa, K562, HL-60, and H1975 cell lines in the MTT and SRB methods. Whilst 201 and 203 showed radical scavenging activity against DPPH ( $\mathrm{IC}_{50} 5.66$ and $6.67 \mu \mathrm{M}$, respectively). None of these metabolites exhibited antimicrobial activities against E. coli, E. aerogenes, P. aeruginosa, B. subtilis, and C. albicans [95]. The newly isolated benzopyrone, clapone (192), had no $\alpha$-glycosidase inhibitory effect and no cytotoxic activity towards SGC-7901, K562, Hela, and BEL-7042 cell lines in the MTT assay [67]. Furthermore, $\mathbf{1 8 6}$ and 205 displayed no cytotoxic effect $\left(\mathrm{IC}_{50}>10 \mu \mathrm{M}\right)$ versus HeLa cell line in the MTT assay, as well as no antibacterial potential towards S. aureus, B. cereus, E. coli, V. alginolyticus, V. parahemolyticus, and MR S. aureus (conc. $20 \mu \mathrm{M}$ ) in the microplate assay [88]. C. halotolerans GXIMD 02502 associated with the coral Porites lutea yielded a new benzopyranone derivative, coniochaetone K (196) with unusual C-8 carboxyl, along with 194, 195, 197, and 198 that were evaluated for their cytotoxicity versus 22RV1, C4-2B, and RWPE-1 cell lines (Figure 24). 
<smiles>[R]Oc1cc(O)c2c(c1-c1c(OC)cc(O)c3c(O)c4c(cc13)C[C@@H](CC(=O)OC)OC4=O)C(=O)C(=O)C(CC(O)CC(=O)OC)=C2</smiles><smiles>[R]c1cc(O)c2c(c1-c1c(OC)cc(O)c3c(O)c4c(cc13)C[C@@H](CC(=O)OC)OC4=O)C(=O)C1C(=O)O[C@H](CC(=O)OC)CC1=C2</smiles>

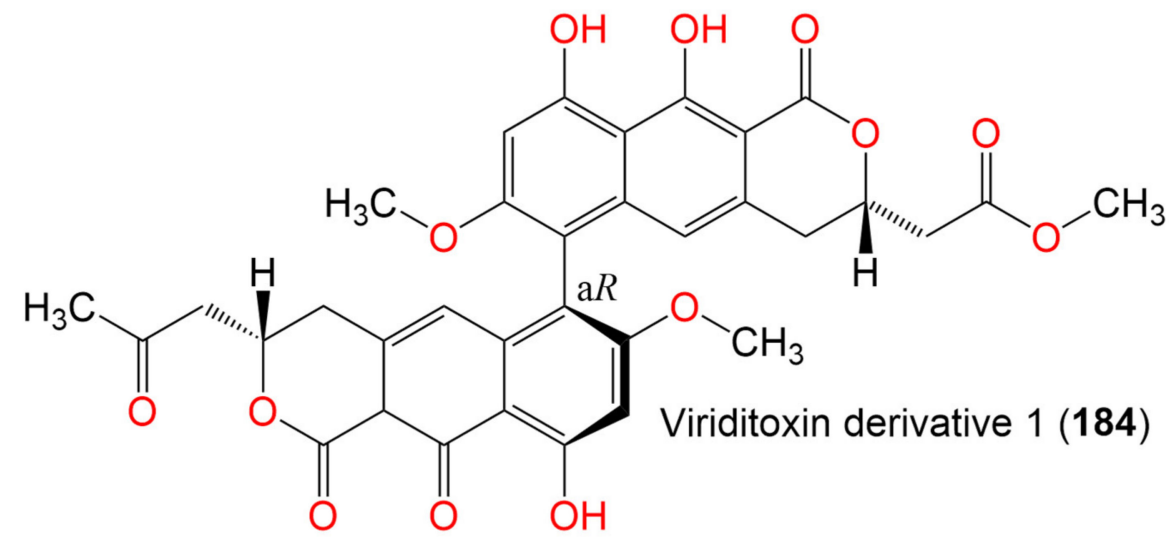<smiles>[R]c1cc(O)c2c(c1-c1c(OC)cc(O)c3c(O)c4c(cc13)C[C@@H](CC(=O)OC)OC4=O)C=C1C[C@@H](CC(C)=O)OC(=O)C1C2=O</smiles>

Figure 23. Binaphthopyrones 182-185. 
<smiles>C[C@H]1CC(=O)c2c(O)cccc2O1</smiles>

(2S)-5-Hydroxy-2methyl-chroman -4-one (186)<smiles>C[C@@H]1CC(=O)c2c(O)cccc2O1</smiles>

(R)-5-Hydroxy-2methylchroman4-one (187)<smiles>CC(CO)OC(C)Oc1cc(O)c2c(c1)O[C@@H](C)CC2=O</smiles>

(2R)-7-O- $\alpha$-D-Ribofuranosyl-5hydroxy-2-methylchroman-4one (188)

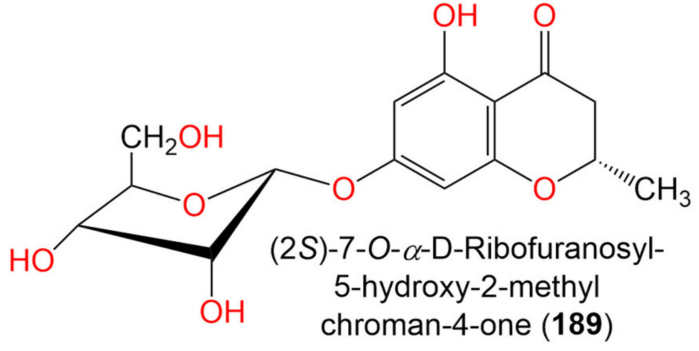<smiles>CCOCC=C(O)O</smiles>

( \pm )-5,7-Dihydroxy-2-methyl chroman-4-one (190)<smiles>Cc1ccc2c(=O)c3c(oc2c1)CCC3=O</smiles><smiles>Cc1cc(=O)c2c(O)cccc2o1</smiles>

5-Hydroxy-2-methyl-4Hchromen-4-one (191)<smiles>C/C=C/c1cc(=O)c2c(C)cc(O)cc2o1</smiles>
Clapone (192)

Coniochaetone B (195)

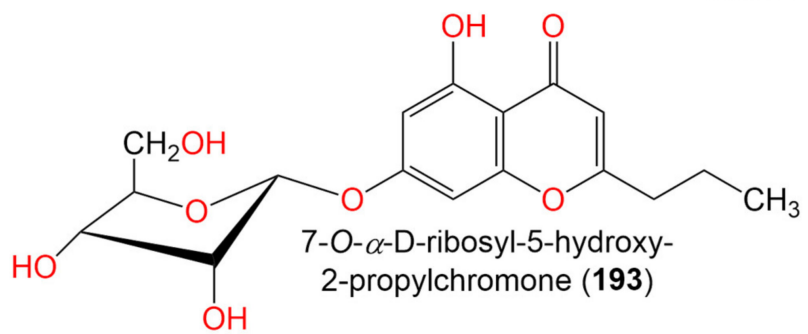<smiles>O=C(O)c1cc(O)c2c(=O)c3c(oc2c1)CC[C@H]3O</smiles><smiles>COC(=O)[C@]1(O)c2c(oc3cc(C)cc(O)c3c2=O)CC[C@H]1O</smiles><smiles>COC(=O)OCCCCC(C)(C)C</smiles>

Figure 24. Benzopyrone derivatives 186-198.

Among them, 194 and 196 revealed notable cytotoxicity versus 22RV1 cells (inhibition $67.4 \%$ and $64.6 \%$, respectively, at conc. $10 \mu \mathrm{M}$ ). On the other hand, they exhibited nearly no cytotoxic effect versus RWPE-1 and C4-2B cells [92]. Bai et al. reported that 206 prohibited the growth of $\mathrm{H}$. armigera Hubner newly hatched larvae ( $\mathrm{IC}_{50} 100 \mu \mathrm{g} / \mathrm{mL}$ ), compared to azadirachtin ( $\mathrm{IC}_{50} 25 \mu \mathrm{g} / \mathrm{mL}$ ) [71]. Further, it showed moderate antibacterial potential versus S. aureus (MIC $6.25 \mu \mathrm{g} / \mathrm{mL}$ ), compared with ciprofloxacin (MIC $0.39 \mu \mathrm{g} / \mathrm{mL}$ ) [71] Cladosporin C (207) did not have obvious anti-biofilm activity towards S. aureus, E. coli, and B. subtilis [36]. On the other hand, it showed moderate toxicity towards brine shrine naupalii ( $\left.\mathrm{LC}_{50} 49.9 \mu \mathrm{M}\right)$, compared to toosendanin $\left(\mathrm{LC}_{50} 21.2 \mu \mathrm{M}\right)$ in the brine shrimp lethality assay [36]. Furthermore, 210 possessed no anti-allergic effect $\left(\mathrm{IC}_{50}>200 \mu \mathrm{M}\right)$ on RBL-2H3 cells, in comparison to loratadine ( $\left.\mathrm{IC}_{50} 35.01 \mu \mathrm{M}\right)$ using fluorometric assay [75]. $\alpha$-Pyrone derivatives 211-213 were separated from $C$. herbarum isolated from the sponge 
Aplysina aerophoba (Figure 25). Compounds $\mathbf{2 1 1}$ and $\mathbf{2 1 2}$ had activity towards Artemia salina (conc. $100 \mu \mathrm{g}$ and $50 \mu \mathrm{g}$ ) with mortality rates 85 and $75 \%$ and 80 and $65 \%$, respectively, while $\mathbf{2 1 3}$ did not have any activity. Besides, $\mathbf{2 1 3}$ showed growth inhibitory activity towards Spodoptera littoralis larvae (7 and 33\% at conc. 250 and 100 ppm, respectively) [96]. However, 211-213 did not show any noticeable antimicrobial activity in the agar plate diffusion assay [96].<smiles>C[C@@H]1Oc2cccc(O)c2[C@@H](O)[C@@H]1O</smiles>

$(2 S, 3 S, 4 R)-2-$ Methylchroman3,4,5-triol (200)<smiles>CO[C@H]1C[C@H](C)Oc2cccc(O)c21</smiles>
$(2 S, 4 S)-4-M e t h o x y-2-$ methylchroman-5-ol (201)<smiles>C[C@@H]1C[C@H](O)c2c(O)cccc2O1</smiles>
$(2 R, 4 S)$-2-methyl chroman-4,5-diol (204)

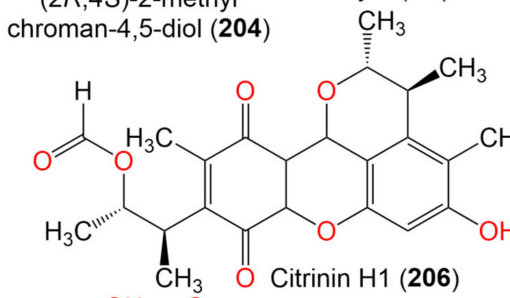<smiles>COc1cc(=O)oc(/C=C/C(=O)O)c1C</smiles>
$\mathrm{H}_{3} \mathrm{C}$<smiles>C[C@@H]1CC(=O)Oc2cccc(O)c21</smiles>
(S)-5-Hydroxy-4-methy chroman-2-one (208) Isoochracinic acid (209)<smiles>CO[C@H]1C[C@@H](C)Oc2cccc(O)c21</smiles>

$(2 R, 4 R)-3,4-$ Dihydro-4-methoxy-<smiles>C[C@H]1C[C@@H](O)c2ccccc2O1</smilesOH

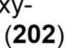

$$
\text { chroman-4,5-diol (203) }
$$<smiles>Cc1c(O)cc2c3c1C(C)C(C)OC3CC(=O)O2</smiles>

Cladosporin C (207)<smiles>CCCCOc1oc(=O)cc(OC)c1/C=C/C(=O)O</smiles><smiles></smiles>

Figure 25. Benzopyrone 199-210 and pyrone (211-214) derivatives.

\subsection{Lactones, Cyclohexene, and Azaphilone Derivatives}

In 2020, He et al. purified 216 from C. cladosporioides that possessed no anti-allergic effect $\left(\mathrm{IC}_{50}>200 \mu \mathrm{M}\right)$ on RBL-2H3 cells, in comparison to loratadine ( $\left.\mathrm{IC}_{50} 35.01 \mu \mathrm{M}\right)$ using fluorometeric assay [75]. The mangrove plant $C$. tagal associated-fungus Cladosporium sp. JJM22 produced new cyclohexene derivatives, cladoscyclitols A-D (218-221) (Figure 26). Compound $219\left(\mathrm{IC}_{50} 2.95 \mu \mathrm{M}\right)$ revealed potent $\alpha$-glucosidase inhibitory activity, compared to acarbose ( $\left.\mathrm{IC}_{50} 2.35 \mu \mathrm{M}\right)$ in the colorimetric assay [97]. On the other hand, it had no antimicrobial potential towards S. aureus ATCC-6538, E. coli ATCC-25922, B. cereu ATCC6633, V. alginolyticus ATCC-3787, V. Parahemolyticus ATCC-17802, or MRSA CMCC-B-63303 
in the micro-plate assay [97]. Perangustols A (223) and B (224), representing new azaphilone epimers, together with bicyclic diol (225) were separated from sea sediment-associated C. perangustum FS62 fungus. They had no observable cytotoxic activity towards SF-268, NCI-H460, MCF-7, and HepG-2 (Conc. $100 \mu \mathrm{M}$ ) in the SRB assay [87].<smiles>C[C@]1(O)CCOC(=O)C1</smiles>

(R)-Mevalonolactone (215)<smiles>CCC/C=C/C1=C(CO)[C@H](O)[C@@H](O)[C@H](O)[C@H]1OC(=O)[O-]</smiles><smiles>CCCCCC1=C(CO)[C@H](O)[C@H](O)C[C@H]1O</smiles><smiles>CCCC(=O)C1=C(O)CC(O)CC1=O</smiles>

2-Butyryl-3,5-dihydroxycyclo hex-2-enone (222)<smiles>CC1=CC2=CC(=O)[C@](C)(O)[C@H](O)[C@H]2CO1</smiles>

Perangustol B (224)<smiles>C/C=C(\C)[C@H]1OC(=O)C(C)(C)[C@H](O)[C@@H]1C</smiles>

Helicascolide A (217)<smiles>CCCCCC1=C(CO)[C@H](O)[C@@H](OC(=O)O)[C@H](O)[C@H]1O</smiles><smiles>CCCCCC1=C(CO)[C@H](O)[C@@H](O)[C@H](O)[C@H]1O</smiles><smiles>CC1=CC2=CC(=O)[C@](C)(O)[C@H](O)[C@H]2CO1</smiles>

Perangustol A (223)<smiles>CC1=CC2=C(CO1)C(=O)[C@](C)(O)[C@H](O)C2</smiles>

Figure 26. Lactone (215-217), cyclohexene (218-222), and azaphilones (223-225) derivatives.

\subsection{Phenolics and Other Aromatic Compounds}

In the DPPH assay, 233 and 235 showed $\mathrm{DPPH}$ radical scavenging activity $\left(\mathrm{IC}_{50} \mathrm{~s}\right.$ 0.24 and $2.65 \mu \mathrm{M}$, respectively), in comparison to ascorbic acid ( $\left.\mathrm{IC}_{50} 3.29 \mu \mathrm{M}\right)$. Further, none of these compounds had antimicrobial potential versus $P$. aeruginosa, E. aerogenes, B. subtilis, E. coli, and C. albicans [95]. The metabolites 232, 238, and 249 were separated from EtOAc extract of Cladosporium sp. F14 isolated from seawater and investigated for their anti-larval activity (conc. $50 \mu \mathrm{g} / \mathrm{mL}$ ) towards B. neritina and B. amphitrite larvae in the settlement inhibition assays [60] (Figure 27). Compound 232 had weak larvae settlement inhibition towards $B$. neritina and B. Amphitrite, respectively, whereas 238 and 249 showed weak inhibitory effects towards $B$. amphitrite and B. neritina larvae, respectively. In another larval settlement bioassay, 232, 238, and 249 inhibited $B$. neritina larval settlement $\left(\mathrm{EC}_{50}\right.$ $11.51,102.23$, and $77.85 \mu \mathrm{g} / \mathrm{mL}$, respectively) and $B$. amphitrite larval settlement $\left(\mathrm{EC}_{50}\right.$ 84.28, 53.65, and $9.18 \mu \mathrm{g} / \mathrm{mL}$, respectively). The larval settlement $\mathrm{EC}_{50}$ values of 249 towards B. amphitrite and 232 towards B. neritina were less than the US Navy program established standard requirement $\left(\mathrm{EC}_{50} 25.0 \mu \mathrm{g} / \mathrm{mL}\right)$, revealing the potential of 232 and 249 as antifouling agents [60]. Furthermore, 232 obviously prohibited L. hongkongensis 
growth (IZD $8 \mathrm{~mm}$ and MIC $80 \mu \mathrm{g} / \mathrm{mL}$ ), compared to streptomycin (MIC $250 \mu \mathrm{g} / \mathrm{mL}$ ) [60]. The ribofuranose phenol derivative, 239 isolated Cladosporium sp. JJM22 associated with the mangrove plant $C$. tagal had no cytotoxic effect $\left(\mathrm{IC}_{50}>10 \mu \mathrm{M}\right)$ versus HeLa cell line in the MTT assay, compared to epirubicin [88].<smiles>O=C(O)CCc1ccccc1P(O)OC(=O)[O-]</smiles><smiles>O=C(O)c1cc(O)cc(O)c1</smiles>

$\alpha$-Resorcylic acid (229)<smiles>Cc1ccc(C(=O)O)cc1</smiles>

$P$-Toluic acid (227)<smiles>O=C(O)Cc1ccccc1</smiles><smiles>O=C(O)[C@H](O)Cc1ccccc1</smiles>

L- $\beta$-Phenyllactic acid (228)<smiles>O=C(O)Cc1ccc(O)cc1</smiles>

P-Hydroxyphenylacetic<smiles>O=C(O)/C=C/c1ccccc1</smiles>

3-Phenyl-2-propenoic acid (232)<smiles>C[C@H](CC(=O)O)Oc1cccc(O)c1O</smiles>

3-(2,3-Dihydroxy phenoxy) butanoic acid (233)<smiles>OCCc1ccc(O)cc1</smiles>

$P$-Hydroxyphenylethyl alcohol (236)<smiles>OCc1ccc(O)cc1</smiles><smiles>[124IH]</smiles>

$$
\text { acid (231) }
$$<smiles>COC(=O)c1ccc(O)cc1</smiles><smiles>COC(=O)C[C@H](C)Oc1cccc(O)c1O</smiles>

$P$-Hydroxy benzoic acid methyl ester (234) $\mathrm{H}_{3}$

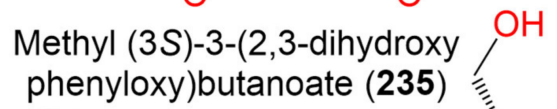

phenyloxy)butanoate (235)<smiles>OC[C@H]1O[C@@H](O)[C@@H](O)[C@@H]1O</smiles>

O'

4-O- $\alpha$-D-Ribofuranose-3-hydroxymethyl -2-pentyl- phenol (239)

P-Hydroxybenzyl alcohol (237)<smiles>CCCCCc1c(O)ccc(Cl)c1CO</smiles>

\section{4-O- $\alpha$-D-Ribofuranose-2-} pentyl-3-phemethylol (240)<smiles>CC(=O)c1cc(C)c(O)c(C)c1O</smiles>

Clavatol (241)

Figure 27. Phenolics 226-241.

Additionally, it exhibited no noticeable antibacterial potential towards S. aureus, B. cereus, E. coli, V. alginolyticus, V. parahemolyticus, and MR S. aureus (conc. $20 \mu \mathrm{M}$ ) in the microplate assay [88]. The new ribofuranose phenol derivative, $240\left(\mathrm{IC}_{50} 2.05 \mu \mathrm{M}\right)$ 
revealed potent $\alpha$-glucosidase inhibitory activity, compared to acarbose $\left(\mathrm{IC}_{50} 2.35 \mu \mathrm{M}\right)$ in the colorimetric assay [97]. On the other hand, it had no antimicrobial potential towards S. aureus ATCC-6538, E. coli ATCC-25922, B. cereus ATCC-6633, V. alginolyticus ATCC-3787, V. Parahemolyticus ATCC-17802, and MRSA CMCC-B-63303 in the microplate assay [97]. Phytochemical investigation of the mycelium extract of the marine-derived fungus Cladosporium sp. associated with Chondria crassicualis red alga resulted in the separation of a phenol derivative, clavatol (241) that exhibited antioxidant capacity $\left(E_{50} 227.0 \mu \mathrm{M}\right)$ more than oxybenzone (sunscreen agent, $\mathrm{ED}_{50} 350 \mu \mathrm{M}$ ) as evident by their UV-A protecting potential [65]. On the other hand, it was inactive towards MDRSA, MRSA, and S. aureus [65]. Fan et al. stated that compounds 242 and 243 exhibited no observable cytotoxic activity towards SF-268, NCI-H460, MCF-7, and HepG-2 (Conc. $100 \mu \mathrm{M}$ ) in the SRB assay [87] (Figure 28). Cladosporin D (247) did not have obvious anti-biofilm activity towards $S$. aureus, E. coli, and B. subtilis [36], while it exhibited significant antioxidant activity ( $\left.\mathrm{IC}_{50} 16.4 \mu \mathrm{M}\right)$, compared with ascorbic acid $\left(\mathrm{IC}_{50} 4.9 \mu \mathrm{M}\right)$. Besides, it showed moderate toxicity towards brine shrine naupalii $\left(\mathrm{LC}_{50} 81.4 \mu \mathrm{M}\right)$, comparing with toosendanin $\left(\mathrm{LC}_{50} 21.2 \mu \mathrm{M}\right)$ in the brine shrimp lethality assay [13].

Compound 248 separated from Cladosporium sp. TPU1507 derived from marine sponge and assessed for inhibitory effect towards PTP1B and TCPTP using enzyme-based assay [68]. It showed an inhibitory effect on TCPTP $\left(\mathrm{IC}_{50} 27 \mu \mathrm{M}\right)$ that was 2 -fold weaker than on PTP1B ( $\left.\mathrm{IC}_{50} 11 \mu \mathrm{M}\right)$ [68]. The new phthalide, herbaric acid (250), separated from $C$. herbarum isolated from Callyspongia aerizusa had no activity towards $A$. salina and HL-60 human leukemia cell line [96]. In addition, the newly separated abscisic acid analog 251 from Cladosporium sp. OUCMDZ-1635 possessed no cytotoxic effect towards MCF-7, HeLa, HCT-116, HeLa, HCT-116, K562, and HL-60. Furthermore, it did not show antibacterial activity (conc. $100 \mu \mathrm{g} / \mathrm{mL}$ ) against B. subtilis, P. aeruginosa, C. perfringens, S. aureus, E. coli, and C. albicans [56]. The new pentenoic acid derivative, 1,1'-dioxine2,2'-dipropionic acid (252) prohibited the growth of $H$. armigera Hubner newly hatched larvae $\left(\mathrm{IC}_{50} 150 \mu \mathrm{g} / \mathrm{mL}\right)$, compared to azadirachtin $\left(\mathrm{IC}_{50} 25 \mu \mathrm{g} / \mathrm{mL}\right)$ [71]. Further, it showed moderate antibacterial potential versus $S$. aureus (MIC $25.0 \mu \mathrm{g} / \mathrm{mL}$ ), compared with ciprofloxacin (MIC $0.39 \mu \mathrm{g} / \mathrm{mL}$ ) [71]. The furan carboxylic acid metabolites, Sumiki's acid (253) and acetyl Sumiki's acid (254) exerted activity towards S. aureus and B. subtilis (IZDs $7 \mathrm{~mm}$ at conc. $5 \mu \mathrm{g} /$ disk), whereas they had no activity towards C. albicans and E. coli [74].

\subsection{Sterols and Terpenes}

A study conducted by $\mathrm{Yu}$ et al. in 2018 led to the separation of a new pregnane; $3 \alpha$-hydroxy-7-ene-6,20-dione (268) and six sterol derivatives: 256, 258, 260, 262, 263, and 267 from gorgonian-associated Cladosporium sp. WZ-2008-0042 [100]. Note that 268 was reported in the same year by Pang et al. as new metabolites with the name cladosporisteroid B from Cladosporium sp. SCSIO41007 associated with Callyspongia sp. [61]. These metabolites $\left(\mathrm{IC}_{50}\right.$ values ranging from 0.11 to $\left.0.17 \mu \mathrm{M}\right)$ revealed antiviral activity against RSV (respiratory syncytial virus) with therapeutic ratio $\left(\mathrm{TC}_{50} / \mathrm{IC}_{50}\right)$ values ranging from 5.18 to 9.92 , in comparison to ribavirin in the neuraminidase inhibition assay. This could be due to their binding to RSV GREs (glucocorticoid response elements) [100]. Moreover, they (conc. $0.1 \mathrm{mg} / \mathrm{mL}$ ) displayed weak to moderate AChEI potential, in comparison to huperzine A and galanthamine using the modified Ellman's method [100]. Further, 268 had no noticeable antibacterial potential towards B. cereus, M. luteus, S. aureus, V. anguillarum E. coli, Shigella dysenteriae, B. subtilis, and V. Parahemolyticus, while 263 was moderately active (MIC $3.13 \mu \mathrm{M}$ ) towards S. dysenteriae [100]. In 2020, He et al. reported that 256, 261, 265, 266, and 268 separated from C. cladosporioides sea sediment-derived fungus possessed no anti-allergic effect on RBL-2H3 cells, in comparison to loratadine using fluorometeric assay [75] (Figures 29 and 30). In 2018, Pang et al. separated new sterol cladosporisteroid A (264) and new pregnanes, cladosporisteroid B (268) and cladosporisteroid C (269), along with 259, 265, and 270 from Cladosporium sp. SCSIO41007 isolated from Callyspongia sp. 
and assessed their antiviral activity towards EV71 and H3N2 using CCK-8 and CPE assays, respectively. Only, 268 ( $\mathrm{IC}_{50} 16.2 \mu \mathrm{M}$ ) had weak activity towards $\mathrm{H} 3 \mathrm{~N} 2$ compared to oseltamivir $\left(\mathrm{IC}_{50} 34.0 \mathrm{nM}\right.$ ). Moreover, they revealed no cytotoxic effect towards K562, MCF-7, and SGC-7901 in the CCK-8 assay [61]. Additionally, 268 was purified from C. sphaerospermum EtOAc fraction by HPLC with the aid of LCMS and assessed for its influence on adipogenesis and lipid metabolism during maturation of adipocyte (Conc. 1.25, 2.5, 5, and $10 \mu \mathrm{M}$ ) using 3T3-L1 preadipocytes [101]. It substantially prohibited lipid accumulation and differentiation of 3T3-L1-preadipocytes into adipocytes, leading to reducing Adipsin (adipocyte marker gene) expression. Further, it significantly upregulated ATGL (lipolytic gene, Conc. 5 and $10 \mu \mathrm{M}$ ) and reduced FASN and SREBP1 (lipogenic genes, conc. 1.25, $2.5,5$, and $10 \mu \mathrm{M})$ expression. Collectively, 268 facilitated lipid metabolism and disrupted adipogenesis via promoting lipolysis and prohibiting lipogenesis [101].<smiles>CC(=O)Cc1cc(O)cc(O)c1</smiles><smiles>CC(=O)c1c(O)cccc1O</smiles><smiles>CC(=O)Cc1cc(O)c(C)c(O)c1</smiles>

$\alpha$-Acetylorcinol (243) 1-(2,6-Dihydroxyphenyl) 1-(3,5-Dihydroxy-4-methylphenyl) ethanone (244) propan-2-one (242)

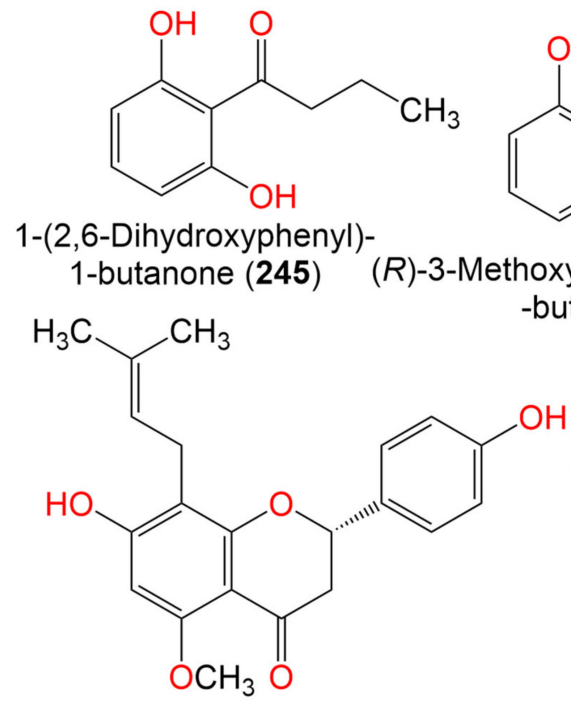

(2S)-7,4'-dihydroxy-5-methoxy-8-<smiles>CO[C@@H](C)CC(=O)c1c(O)cccc1O</smiles><smiles>Cc1c([C@@H](C)[C@H](C)O)cc(O)c(C=O)c1O</smilesbutan-1-one (246)<smiles>CCCCC(CC)COC(=O)c1ccccc1C(=O)OCC(CC)CCCC</smiles>

Bis(2-Ethylhexyl)phthalate (249) $(\gamma, \gamma$-dimethylallyl)-flavanone (248)<smiles>O=C(O)CCC1=COC(CCC(=O)O)=CO1</smiles><smiles>CC1=CC(=O)CC(C)(C)[C@H]1CC/C(C)=C/C(=O)O</smiles>

Cladosacid (251)<smiles>O=C1O[C@H](CO)c2cc(O)cc(O)c21</smiles><smiles>O=C(O)c1ccc(CO)o1</smiles>

Sumiki's acid (253)<smiles>CC(=O)OCc1ccc(C(=O)O)o1</smiles>

Herbaric acid (250)

Figure 28. Phenolics 242-248 and others 249-254. 


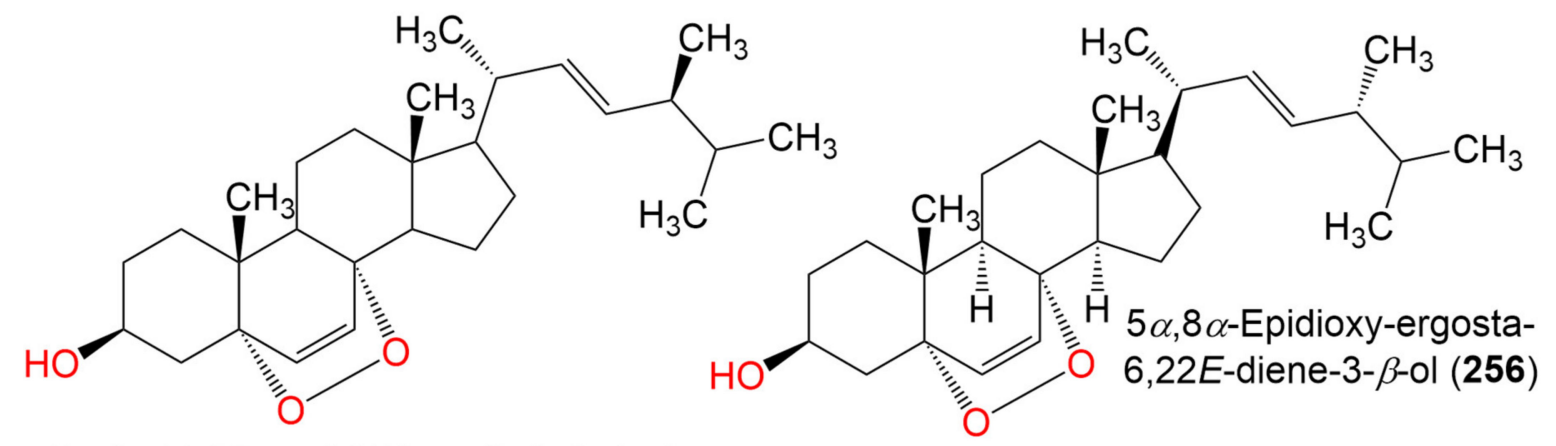

$5 \alpha, 8 \alpha$-Epidioxy-24(R)-methyl-cholesta-

6,22 -diene-3- $\beta$-ol (255)

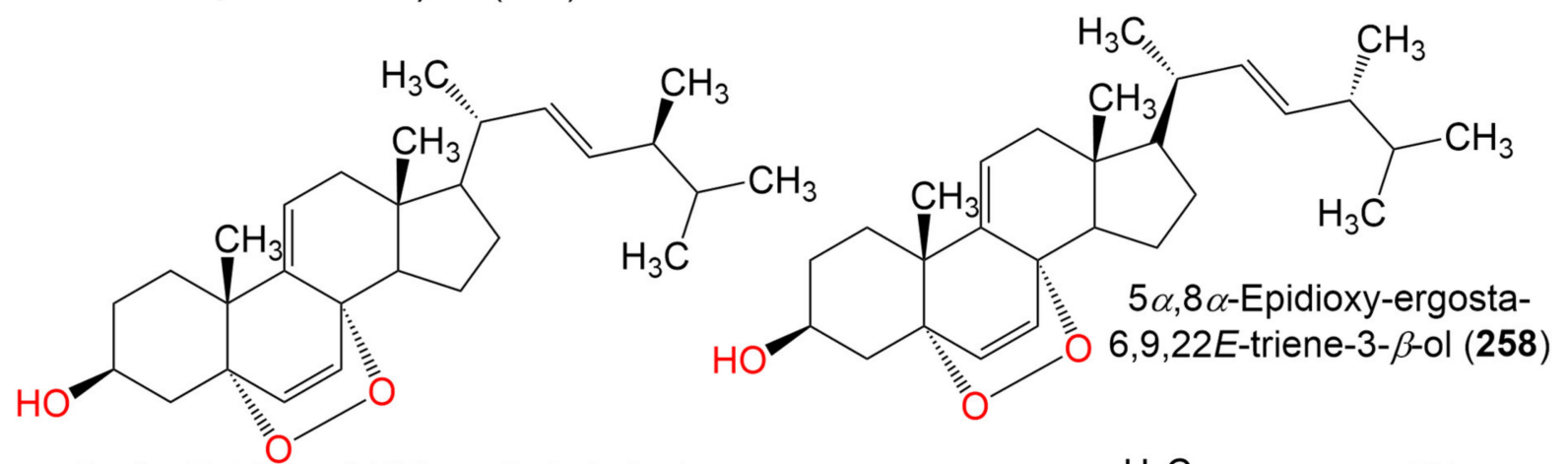

$5 \alpha, 8 \alpha$-Epidioxy-24(R)-methyl-cholesta6,9(11),22-triene-3- $\beta$-ol (257)

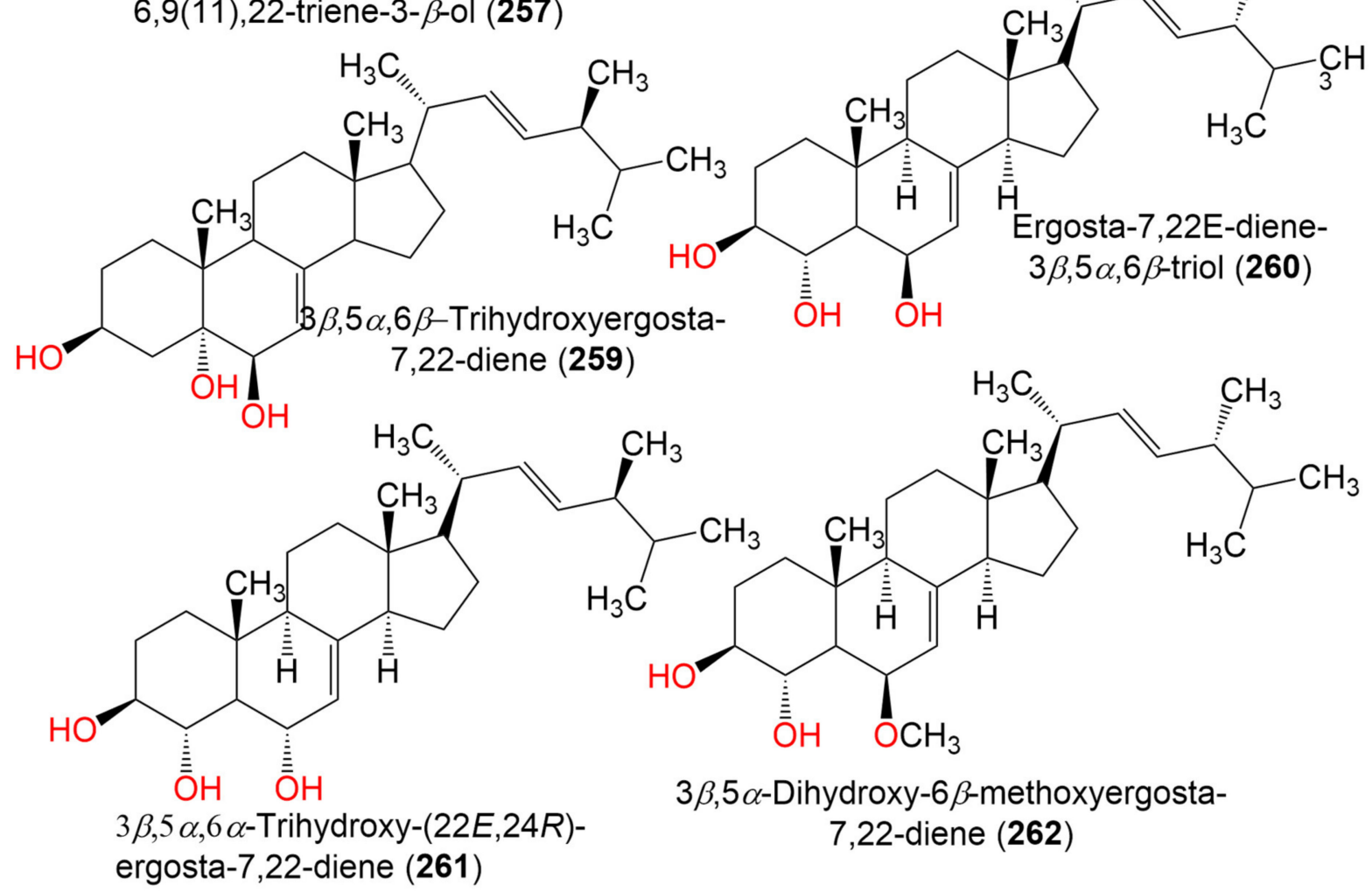

Figure 29. Sterols 255-262. 


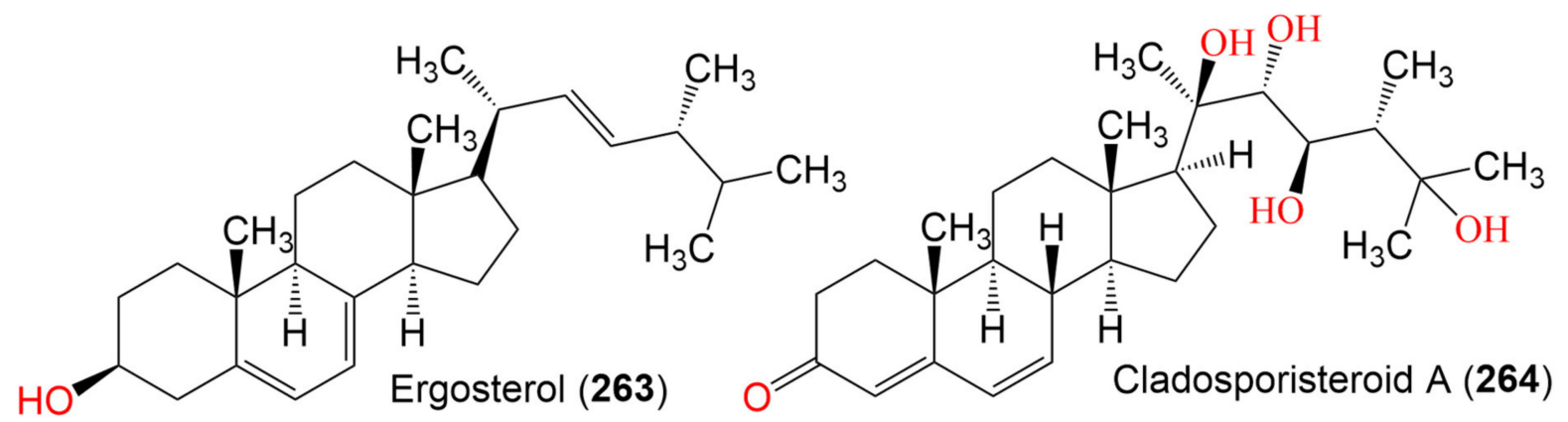<smiles>CC(C)[C@H](C)/C=C/[C@H](C)[C@H]1CC[C@H]2C3=CC(=O)[C@]4(O)C[C@@H](O)CC[C@]4(C)[C@@H]3CC[C@]2(C)[C@H]1C</smiles>

$3 \beta, 5 \alpha, 9 \alpha$-Trihydroxy-(22E,24R)ergosta-7,22-diene-6-one (265)

$3 \beta, 5 \alpha$-Dihydroxy-(22E,24R)ergosta-7,22-diene-6-one (266)<smiles>CC(=O)[C@H]1CC[C@H]2C3=CC(=O)[C@@H]4C[C@@H](O)CC[C@]4(C)[C@H]3CC[C@]12C</smiles>

Stigma-5-en-3-O- $\beta$ glucopyranoside (267)<smiles>COC1(OC)CC[C@@]2(C)[C@H]3CC[C@@]4(C)C(=CC(=O)[C@H]4[C@@H]1C(C)=O)[C@@H]3CC[C@H]2C(C)=O</smiles>

Cladosporisteroid C (296)
$3 \alpha$-Hydroxy-pregna-7-ene-6,20-dione (268)<smiles>CC(=O)[C@H]1CC[C@H]2C3=CC(=O)[C@@H]4CC(=O)CC[C@]4(C)[C@H]3CC[C@]12C</smiles>

Pregn-7-dien-3,6,20-trione (270)

Figure 30. Sterols 263-267 and terpenes 268-270.

\subsection{Alcohols and Aldehydes}

Gallo et al. reported for the first time from fungi the isolation of $\alpha, \beta$-unsaturated aldehydes (271-284) from the culture of Cladosporium sp. isolated from intertidal marine sediment [102] (Figure 31). They exerted antimicrobial activity towards E. coli ATCC-25922, 
B. subtilis ATCC-6633, and C. albicans ATCC-18804 in the agar diffusion method. It is noteworthy that this class of metabolites had been reported formerly from red algae (e.g., Corallina mediterranea and Laurencia papillosa, L. spectabilis, and L. undulata) $[155,156]$. The new aliphatic alcohols, $(2 S, 3 S, 4 E)$-hepta-4,6-diene-2,3-diol (285) and (3E, $8 E, 6 S)$-Undeca3,8,10-trien-1,6-diol (286) were assessed for cytotoxic potential versus HeLa, BEL-7402, HL-60, A549, K562, and H1975 cell lines. Compound 286 had a cytotoxic effect versus H1975 cell line $\left(\mathrm{IC}_{50} 10.0 \mu \mathrm{M}\right)$, compared to ADR $\left(\mathrm{IC}_{50} 0.38 \mu \mathrm{M}\right)$. While both metabolites revealed no antioxidant and antimicrobial capacities [95].

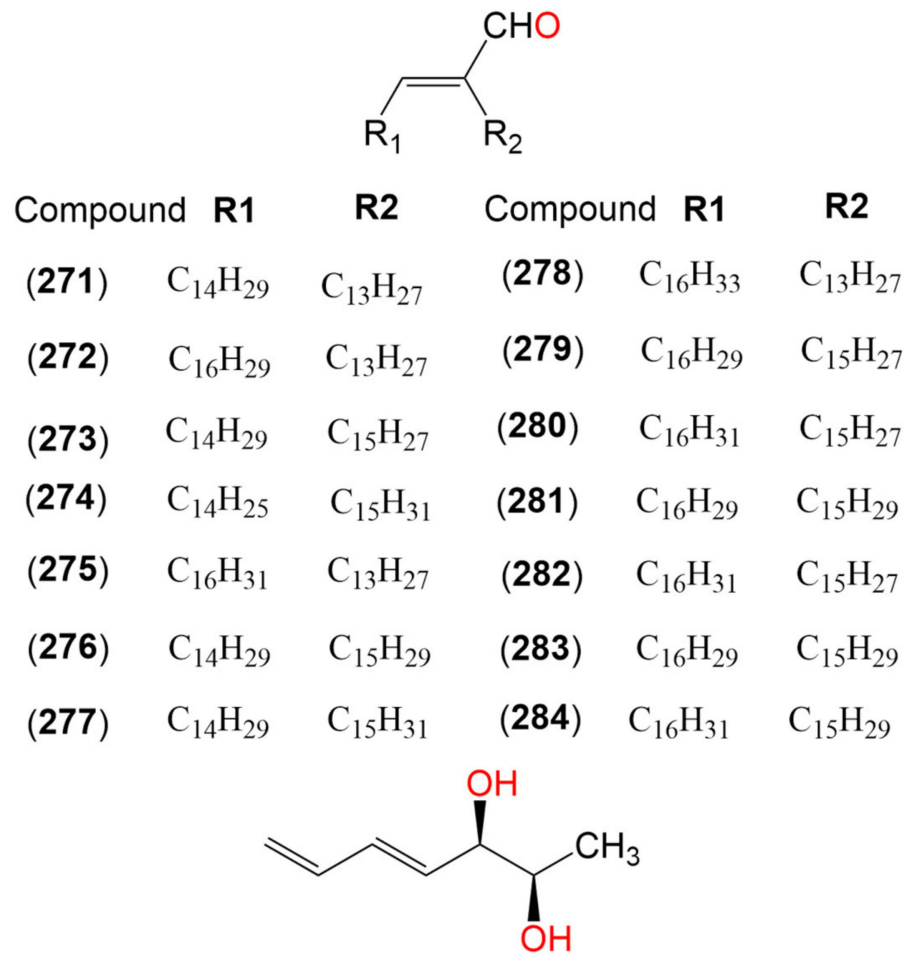

(2S,3S,4E)-Hepta-4,6-diene-2,3-diol (285)<smiles>C=C/C=C/C[C@@H](O)C/C=C/CCO</smiles>

(3E,8E,6S)-Undeca-3,8,10-trien-1,6-diol (286)

Figure 31. Aldehydes (271-284) and alcohols (285 and 286).

\subsection{Bioactivities of Cladosporium Species Extracts}

Ding et al. stated that Cladosporium sp. isolate N5 associated with Porphyra yezoensis red alga did not produce any pathogenic symptoms in the reinfection assay. Further, its EtOAc extract displayed no lethality to $A$. salina and had a moderate antimicrobial activity which indicated that Cladosporium sp. had no toxicity to the aquatic ecosystem and could be applied as a biocontrol agent [59]. In the disc diffusion method, Cladosporium sp. EIODSF 008 EtOAc extract exhibited significant antibacterial potential towards E. coli, M. luteus, and B. subtilis (conc. $100 \mu \mathrm{g} /$ disc) [57]. The EtOAc extract of Cladosporium sp. EN-S01 isolated from Sargassum cinereum brown algae showed anticancer activity towards MCF-7, HeLa, and DU-145 cell lines ( $\mathrm{IC}_{50} 8.46,9.87$, and $98.03 \mu \mathrm{g} / \mathrm{mL}$, respectively). The extract had greater cytotoxic activity and anti-proliferative towards MCF-7 and HeLa cell lines than towards DU-145 [157]. Moreover, the EtOAc extract of C. cladosporioides KT384175 isolated from the seaweed Sargassum wightii possessed remarkable antioxidant potential 
that was comparable to ascorbic acid, as well as significant $\mathrm{Fe}^{3+}$ reducing power that could be referred to its phenolic contents. Moreover, it revealed anti-angiogenic potential as evidenced by the decrease in the number and length of blood vessel branches on CAM (chick chorioallantoic membrane) in-vivo in the CAM assay. Further, C. cladosporioides extract (conc. $1.0 \mathrm{mg} / \mathrm{mL}$ ) had lower wound healing potential than thalidomide (conc. $1.0 \mu \mathrm{g} / \mathrm{mL}$ ) in the in vitro scratch assay using MCF-7 cells [158]. The sea water-derived fungus Cladosporium sp. F14 can produce antifouling and antibiotic metabolites in the existence of xylose or glucose. Significantly, it showed higher antibiotic activity towards M. luteus, P. piscida, Rhodovulum sp., Ruegeria sp., V. fluvialis, and $V$. harveyi in the existence of a sugar carbon source than in its absence in the disc diffusion assay, even though the fungal cells were well-grown under both conditions. Moreover, it possessed antifouling potential as it reduced the attachment of $B$. neritina (bryozoan larvae) in the larval settlement assay [159]. The gold nanoparticles synthesized from C. cladosporioides isolated from the seaweed S. wightii possessed noticeable antimicrobial potential towards E. coli MTCC-118, B. subtilis MTCC-441, S. aureus MTCC-7443, P. aeruginosa MTCC-424, and A. niger MTCC-281 with the highest growth inhibition towards $S$. aureus (IZD $12 \mathrm{~mm}$ ) and least activity against B. subtilis (IZD $9.5 \mathrm{~mm}$ ), compared to ampicillin (IZDs 15 and $12 \mathrm{~mm}$, respectively) in the well diffusion method. Furthermore, they also had significant antioxidant potential comparable to ascorbic acid in the DPPH assay and moderate effectiveness in reducing power assay [160]. Ameen et al. reported that the AgNPs synthesized from C. halotolerans biomass isolated from the marine debris collected around Tarout Island showed a significant free radical scavenging effect (\%inhibition $78 \%$ within 30 min incubation) in the DPPH assay. Moreover, it exhibited cytotoxic potential towards MCF-7 ( $\mathrm{IC}_{50} 34.27 \mu \mathrm{L} / \mathrm{mL}$ ), compared to cisplatin ( $\mathrm{IC}_{50} 17.69 \mu \mathrm{L} / \mathrm{mL}$ ) in the MTT assay, as well as an antifungal effect against $A$. niger (\%inhibition 70 and $45 \%$ at conc. 1000 and 500 ppm, respectively) in the broth dilution method [161].

From the comprehensive review of the available literature, it was noticed that $C$. phlei (causal agent of Timothy leaf spot disease) and C. cucumerinum (causal agent of scab disease of many Cucurbitaceae plants) were isolated mainly from plant sources [162-165]. These species produced perylenequinone derivatives as major metabolites which are responsible for pigmentation and discolorations of the leaves [162,165]. Additionally, cotylenins, plant growth regulators were isolated from an unidentified Cladosporium species [166-170]. However, tetralones, seco-acids, macrolides, diketopiperazines, alkaloids, and tetramic acid derivatives were reported mainly from marine-associated Cladosporium species.

\section{Conclusions}

Numerous structurally diverse biometabolites are discovered from marine-derived fungi that represent a rich library for the development of drug lead. Marine-associated Cladosporium species are of biotechnological and industrial relevance and could be considered as substantial enzyme producers. Their enzymes are active in harsh conditions such as extremely low temperatures and high salinity. Therefore, they can be utilized in various industrial and biotechnological applications. Besides, these species were found to be a wealthy pool covering a wide array of metabolites with various bioactivities. Over the past 22 years, 286 metabolites have been separated from marine-associated Cladosporium species isolated from various marine samples, including mangrove, sediment, sponges, corals, gorgonians, algae, bivalves, hydroids, and others (Figure 32).

More than $75 \%$ of these metabolites have been reported from unidentified Cladosporium sp. (175 metabolites, 61\%) and C. cladosporioides (53 metabolites, 18.5\%) (Figure 33).

The results revealed that alkaloids, macrolides, tetramic acid and pyrone derivatives, and phenolics are the major metabolites reported from this marine-associated fungal species (Figure 34). They could be privileged and useful candidates for chemists and biologists to design structurally novel and pharmacologically important compounds for various diseases. 


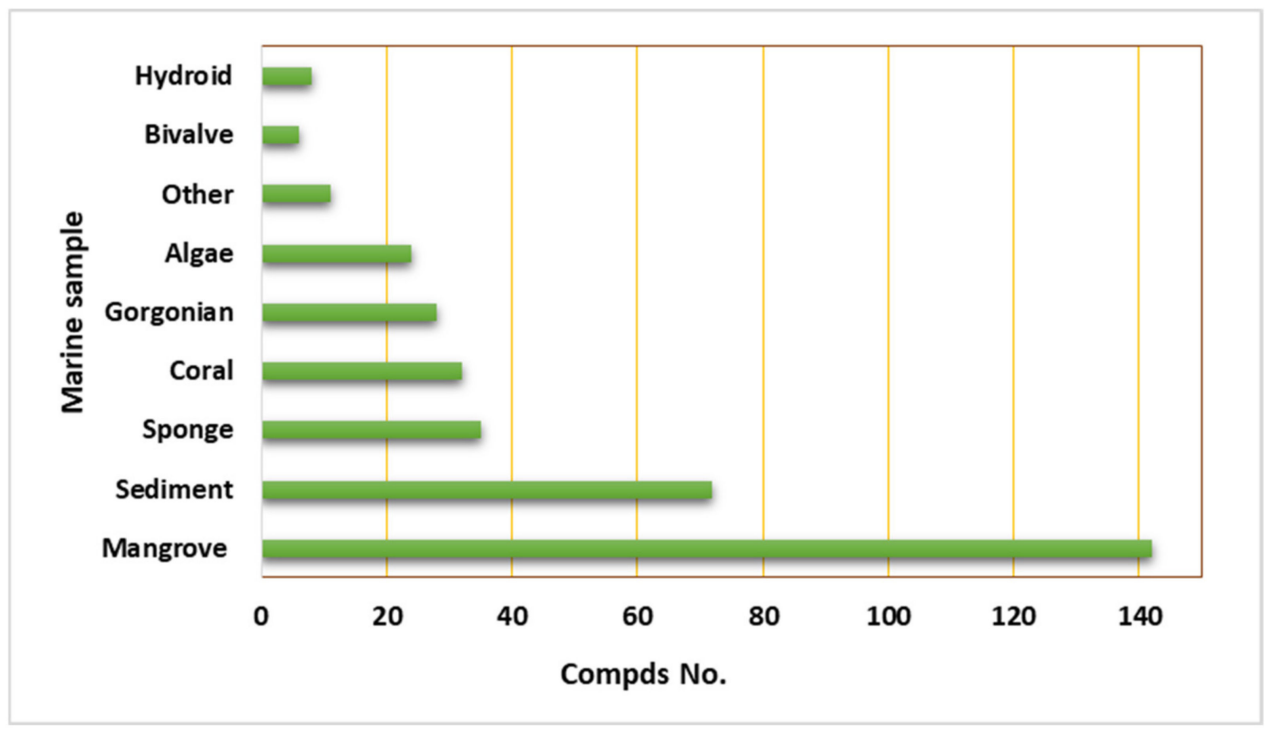

Figure 32. Number of compounds separated from Cladosporium species isolated from various marine samples.

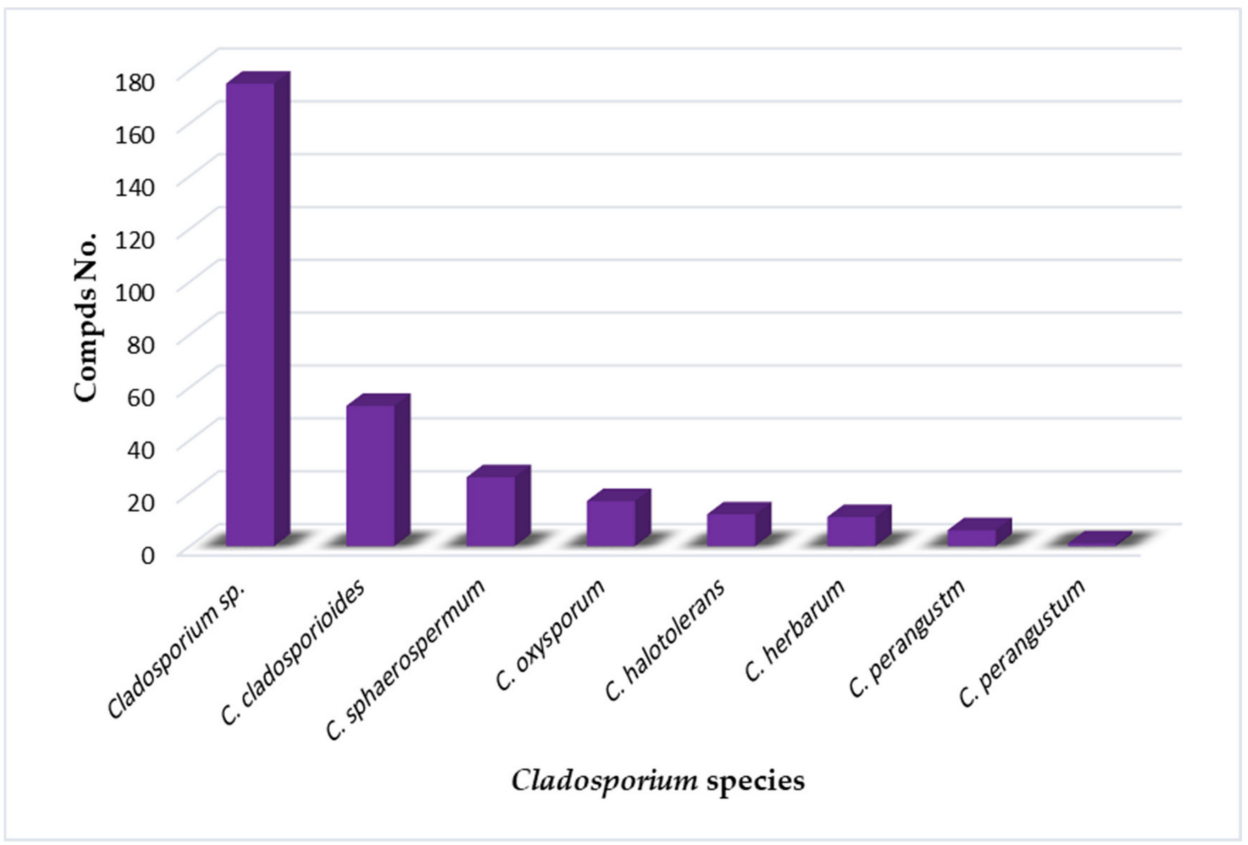

Figure 33. Number of compounds separated from marine-derived Cladosporium species.

Although the structural diversity of these metabolites, they were insufficiently evaluated for their bioactivities. Most of them had been assessed for their antimicrobial, cytotoxicity, antiviral, and insecticidal activities (Figure 35).

Figure 36 illustrated the prominent activities of each class of secondary metabolites.

However, there are limited studies that focus on the mechanism of action of these metabolites. Many of the tested metabolites possessed no noticeable efficacy in some of the tested activities. Therefore, estimation of other potential bioactivities and derivatization of these metabolites, as well as the mechanistic and in vivo studies of the active metabolites should clearly be the target of future research. 


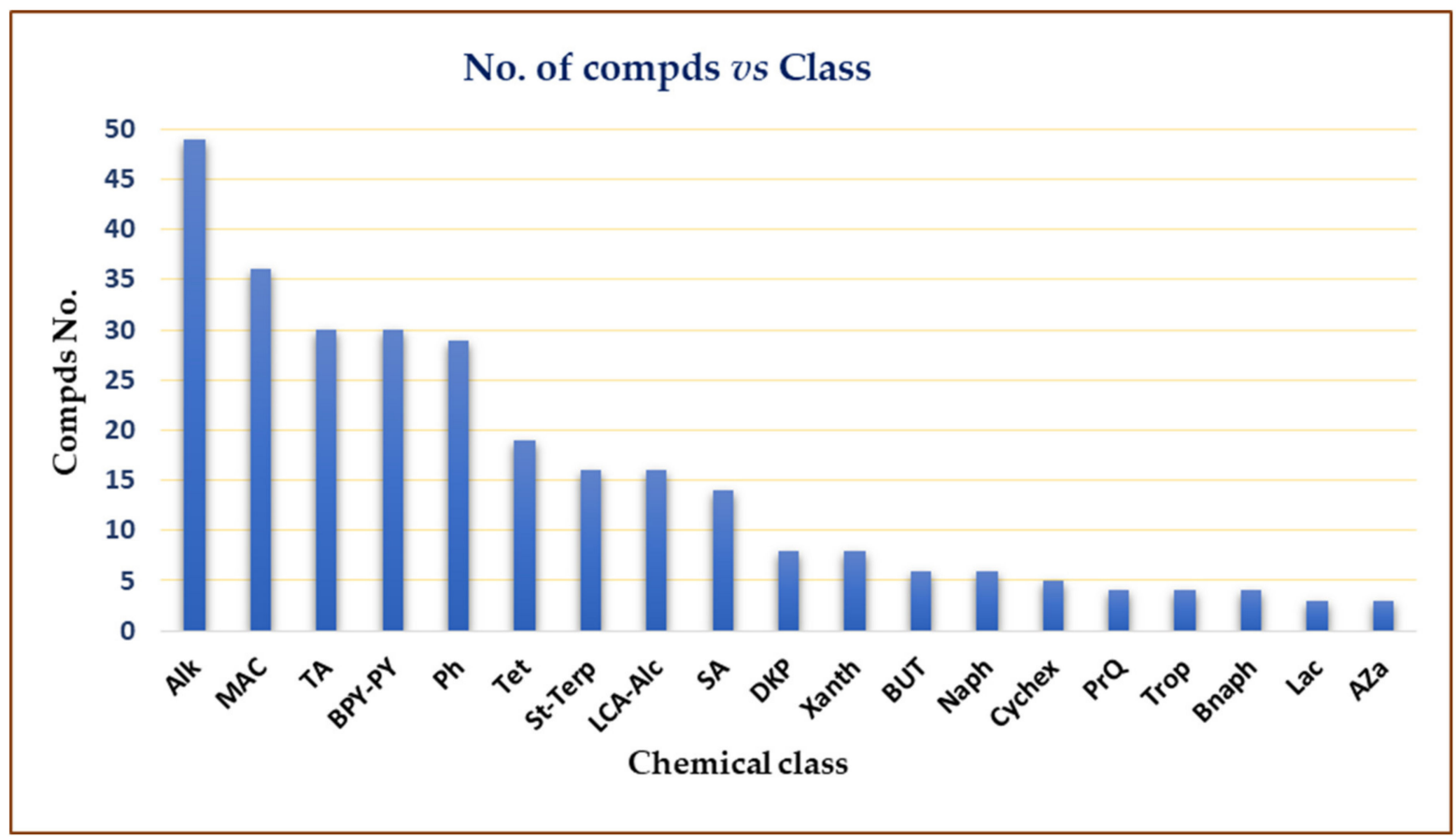

Figure 34. Number of metabolites from each class of natural products.

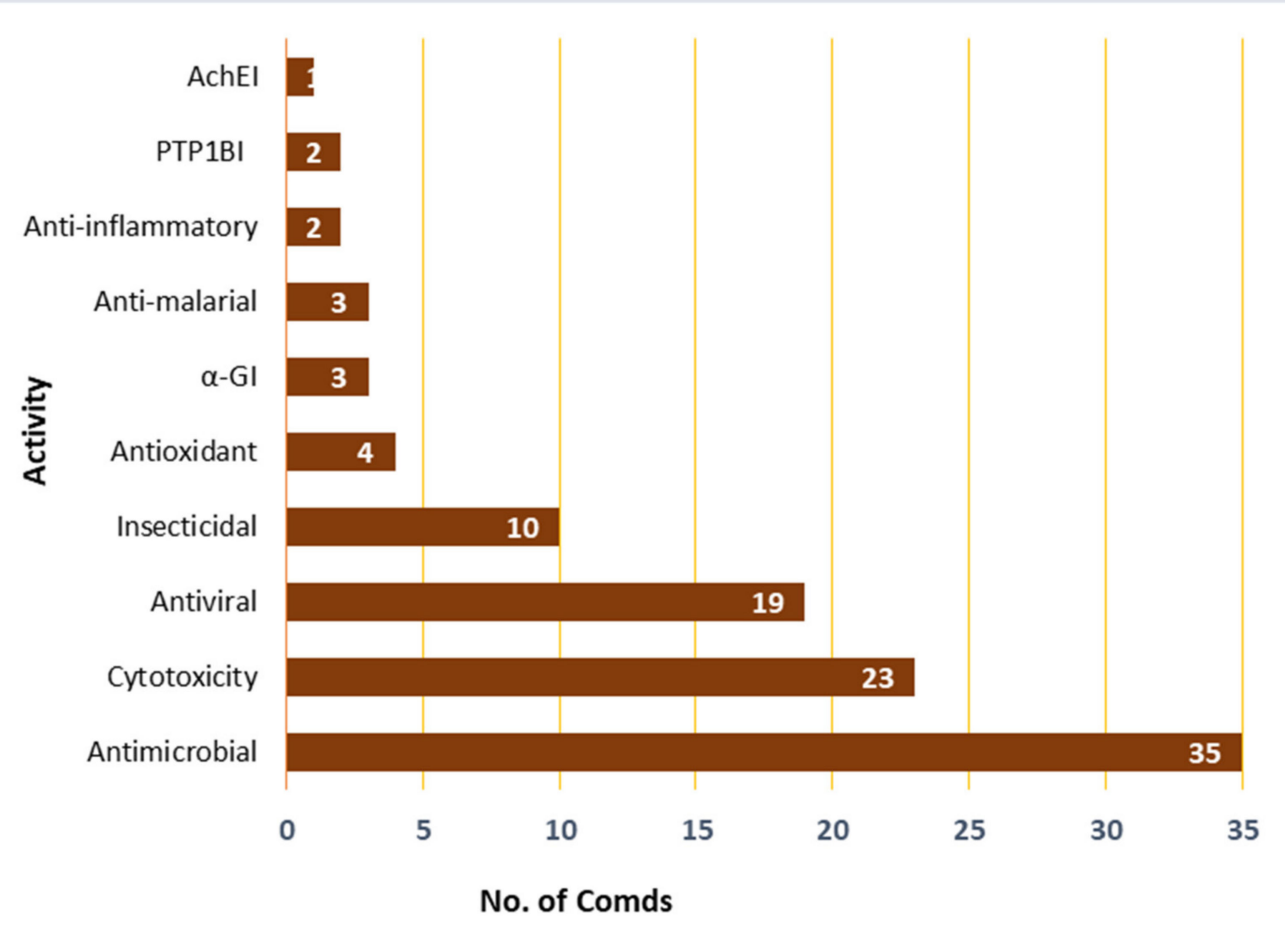

Figure 35. Number of bioactive compounds in each tested activity. 


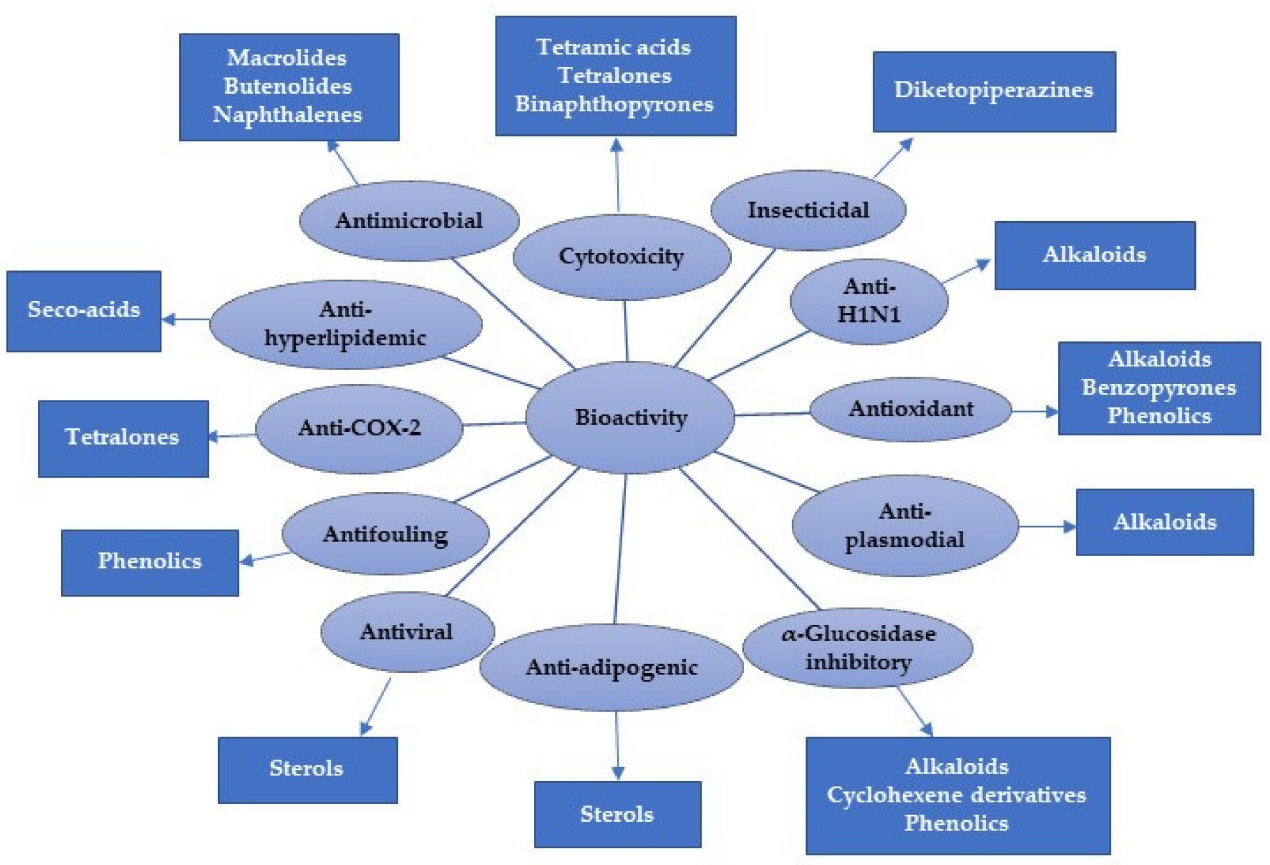

Figure 36. Prominent activities of each class of metabolite from Cladosporium species.

\section{Strategies for Activating Silencing Gene Clusters}

Growing evidence has revealed that the activation of silent gene clusters has the potential to significantly enhance the discovery of new natural metabolites of high-therapeutic leads. Different strategies to awake the silent biosynthetic gene clusters of Cladosporium species such as co-cultivation of organisms and elicitors epigenetic, as well as, modifiers can be applied [171-174]. The production of secondary metabolites (SMs) is affected by cultivation media, environment, and conditions $[171,175]$. Therefore, manipulating the culture conditions can improve the outputs from living organisms. Small changes in the growth media composition can induce not only variation in the amount of SMs, but also the production of a completely different pattern of molecules [171-173]. OSMAC (one strain many compounds) is a form of strain improvement that summarized the ability of single strains to produce different compounds when growing under different conditions e.g., aeration rate, media composition, type of culturing vessel, or a combination of these factors [174-176]. Challenging the fungi with external cues or chemicals has been shown to enhance the SMs production. Antibiotics have been widely reported as elicitors that can activate a broad spectrum of silent BGCs [171-174]. The co-cultivation of strains of the same or different species has been shown to represent a promising strategy for the activation of silent BGCs that enhances the production of SMs and discovery of new bioactive SMs $[171,172,177]$. Activation of silent biosynthetic gene clusters (BGCs) by quorum sensing class of signaling molecule is another strategy that has been shown to dramatically increase SMs production [171-173]. Engineering strains to circumvent the regulatory systems has the potential to free silent BGCs from their locked-in state and result in a significantly enhancement of SMs production. This can be done through various ways such as ribosome and polymerase engineering, an awakening of the genes encoding transcriptional regulatory proteins, and deletion or deactivation of the suppressor proteins. Another approach is the insertion of inducible artificial promoters to drive the expression of the silent genes $[171,172,178]$. Modulating epigenetic control also plays a role in the expression of silent gene clusters linked to natural product expression $[173,179]$.

Author Contributions: G.A.M. and S.R.M.I. contributed equally to this work. All authors have read and agreed to the published version of the manuscript. 
Funding: The authors extend their appreciation to the Deputyship for Research \& Innovation, Ministry of Education in Saudi Arabia for funding this research work through the project number IFPRP: 50-166-1442 and King Abdulaziz University, DSR, Jeddah, Saudi Arabia.

Institutional Review Board Statement: Not applicable.

Informed Consent Statement: Not applicable.

Acknowledgments: The authors extend their appreciation to the Deputyship for Research \& Innovation, Ministry of Education in Saudi Arabia for funding this research work through the project number IFPRP: 50-166-1442 and King Abdulaziz University, DSR, Jeddah, Saudi Arabia.

Conflicts of Interest: The authors declare no conflict of interest.

\section{Abbreviations}

[ $\left.{ }^{3} \mathrm{H}\right] \mathrm{PDBu}:\left[{ }^{3} \mathrm{H}\right]$ Phorbol-12,13-dibutyrate; 22RV1: Human prostatic cancer cell line; 3T3-L1; Mouse adipocyte-like cell line; 786-0: Human renal carcinoma cell line; A2780; Human ovarian cancer cell; A375: Melanoma cell line; A549: Lung adenocarcinoma epithelial cell line; Ab1: Protooncogene 1, non-receptor tyrosine kinase; ABPA: Allergic bronchopulmonary aspergillosis; ADR: Adriamycin; ATGL: Adipose triglyceride lipase; ATP: Adenosine triphosphate; Bax/Bcl-2: Apoptosis regulator BAX; Bcap-37: Human breast carcinoma cell line; Bel-7402: Human hepatocellular carcinoma cell line; BGC-823: Human gastric cancer cell line; BT549: Human breast cancer cells line; C4-2B: Human prostatic cancer cell line; C4-2B: Human prostatic cancer cell line; CAM: Chick chorioallantoic membrane assay; CCK8: Cell Counting kit-8; CD25: Cluster of differentiation 25; CD28: Cluster of differentiation 28; CD3: Cluster of differentiation 3; CDK: Cyclin-dependent kinase; CDKI: Cyclin-dependent kinase Inhibitor; CDPSs: Cyclodipeptide synthases; CM: Corrected mortality; COX-2: Cyclooxygenase-2; CPE: Cytopathic effect; DAPI: 4,6-Diamidino-2-phenylindole; DELFIA: Dissociation-enhanced lanthanide fluorescence immunoassay; DKPs: Diketopiperazines; DMSO: Dimethylsulfoxide; DPPH: 1,1-Diphenyl-2-picrylhydrazyl; DU-145: Human prostate cancer cell line; $\mathrm{ED}_{50}$ : Effective dose 50; EGFR: Epidermal growth factor receptor; ERK: Extracellular signal-regulated protein kinase; EtOAc: Ethyl acetate; EtOH: Ethanol; EV71: Enterovirus 71; FASN: Fatty acid synthase; FRAP: Ferric reducing antioxidant power; FSW: Filtered seawater; Fyn: Protooncogene tyrosine-protein kinase; GREs: Glucocorticoid response elements; GW9662: Selective PPAR antagonist for PPAR $\gamma$; H1974: Human colorectal cancer cell line; H3N2: Influenza A virus subtype H3N2; H446: Human small cell lung carcinoma; HCC70: Human breast cancer cells line; HCT-116: Human colon cancer cell line; HCT-15: Human colon cancer cell line; HCT-8: Human colorectal cancer cell line; HeLa: Human epithelioid cervix carcinoma cell line; HeLa S3: Human cervix carcinoma cell line; HepG-2: Human liver cancer cell line; HL-60: Human promyelocytic leukemia cell; HT-29: Human colorectal adenocarcinoma cell line; HTRF: Homogeneous time-resolved fluorescence assay; Huh-7: Differentiated hepatocyte-derived cellular carcinoma cell line; $\mathrm{IC}_{50}$ : Half-maximal inhibitory concentration; IFN $\gamma$ : Interferon $\gamma$; IL-2: Interleukin-2; IZD: Inhibition zone diameter; JNK: c-Jun NH2-terminal kinase; Jurkat: Human T lymphoblastic leukemia cell lines; K562: Human kidney cancer cell line; L02: Human hepatic cancer cell line; L1210: Mouse lymphocytic leukemia cell line; L5178Y: Murine lymphoma cell line; Lac: Laccase; LBD: Ligand binding domain; LC3I, II: Microtubule-associated proteins 1A/1B light chain 3B; $\mathrm{LC}_{50}$ : Lethal concentration 50; LCK: Lymphocyte-specific protein tyrosine kinase; LiP: Lignin peroxidase; LLC-PK1: Epithelial-like pig kidney cell line; LM3: Human hepatocellular carcinoma cell line; LNCap: Human prostate cancer cell line; LPS: Lipopolysaccharide; MCF-7: Breast cancer cell line; MDA-MB-231: Human breast cancer cell line; MDA-MB-435: Human breast cancer cell line; MDA-MB-468: Human breast cancer cell line; MDCK: Madin-Darby canine kidney; MD-MBA-231: Breast adenocarcinoma cell line; $\mathrm{MeOH}$ : Methanol; MGC-803: Human gastric carcinoma cell line; MIC: Minimum inhibitory concentration; MMP: Mitochondrial membrane potential; MnP: Manganese-dependent peroxidase; MOLT-4: T lymphoblast-acute lymphoblastic leukemia; MRCNS: Methicillin-resistant coagulase-negative Staphylococci; mRNA: Messenger ribonucleic acid; MRSA: Methicillin-resistant Staphylococcus aureus; MTT: 3-(4,5-Dimethylthiazol-2-yl)-2,5-diphenyltetrazolium bromide; NAC: N-acetylcysteine; NCI-H460: Human lung carcinoma cell line; NF-kB: Nuclear factor Kappa B; NO: Nitric oxide; NRPSs: Non- 
ribosomal peptide synthetases; NS-398: N-[2-(cyclohexyloxy)-4-nitrophenyl]methanesulfonamide; OA: Oleic acid; OCI-AML3: Acute myeloid leukemia cell line; OSMAC: One strain many compounds; OVCAR-3: Human ovarian Cancer cell line; P0: PAX6 promoter; P1: PAX6 promoter; P21: N-Myccyclin-dependent kinase inhibitor 1A; p21waf1/cip1: Cyclin kinase inhibitor p21; P388: Mouse lymphocytic leukemia cell line; PANC-1: Human Pancreatic cancer cell line; PAX6: Paired box gene 6; PBMC: Peripheral blood mononuclear cell; PC-3: Human prostate carcinoma cell line; PG: Polygalacturonase; $\mathrm{PGF}_{2 \alpha}$ : Prostaglandin $\mathrm{F}_{2 \alpha} ;$ PKA: cAMP-dependent protein kinase; PKC: Protein kinase C; PMA: Phorbol 12-myristate 13-acetate; PME: Pectin methylesterase; PPAR $\gamma$ : Peroxisome proliferator-activated receptor $\gamma$; PPRE: Peroxisome proliferator-activated receptor response element; PTP: Protein tyrosine phosphatase; P $\alpha$ : PAX6 promoter; RHO: Rhodopsin; RSV: Respiratory syncytial virus; RWPE-1: Human normal prostate epithelial cell; SAR: Structure-activity relationship; SF-268: Human glioblastoma cell line; SGC-7901: Human gastric cancer cell line; SH-SY5Y: Human neuroblastoma cell line; SK-BR-3: Human Breast cancer cell line; SMMC-7721: Human hepatoma carcinoma cell line; SPR: Surface plasmon resonance; SRB: Sulforhodamine B; SREBP1: Sterol regulatory element binding transcription factor 1; SW1116: Human colon cancer cell line; SW1990: Human pancreatic adenocarcinoma cell line; T47D: Human breast cancer cell line; TC: Total cholesterol; $\mathrm{TC}_{50}$ : Half toxic concentration; TCF: T cell factor; TCPTP: T Cell protein tyrosine phosphatase; TG: Total triglyceride; TRF: Time-resolved fluorescence; U937: Human myeloid leukaemia cell line; WERI: Retinoblastoma cell line; WST-1: (4-[3-4-Iodophenyl]-2-(4-nitrophenyl)-2H-5-tetrazolio)-1,3-benzene disulfonate); Y-79: Retinoblastoma cell line.

\section{References}

1. Townsed, M.; Davies, K.; Hanley, N.; Hewitt, J.E.; Lundquist, C.J.; Lohrer, A.M. The challenge of implementing the marine ecosystem service concept. Front. Mar. Sci. 2018, 5, 359. [CrossRef]

2. Boeuf, G. Marine biodiversity characteristics. C. R. Biol. 2011, 334, 435-440. [CrossRef] [PubMed]

3. Donato, P.D.; Buono, A.; Poli, A.; Finore, I.; Abbamondi, G.R.; Nicolaus, B.; Lama, L. Exploring marine environments for the identification of extremophiles and their enzymes for sustainable and green bioprocesses. Sustainability 2019, 11, 149. [CrossRef]

4. Bonugli-Santos, R.C.; Dos Santos Vasconcelos, M.R.; Passarini, M.R.; Vieira, G.A.; Lopes, V.C.; Mainardi, P.H.; Dos Santos, J.A.; de Azevedo Duarte, L.; Otero, I.V.; da Silva Yoshida, A.M.; et al. Marine-derived fungi: Diversity of enzymes and biotechnological applications. Front. Microbiol. 2015, 6, 269. [CrossRef] [PubMed]

5. Varrella, S.; Barone, G.; Tangherlini, M.; Rastelli, E.; Dell'Anno, A.; Corinaldesi, C. Diversity, Ecological Role and Biotechnological Potential of Antarctic Marine Fungi. J. Fungi 2021, 7, 391. [CrossRef] [PubMed]

6. Lindequist, U. Marine-derived pharmaceuticals-challenges and opportunities. Biomol. Ther. 2016, 24, 561-571. [CrossRef] [PubMed]

7. Carroll, A.R.; Copp, B.R.; Davis, R.A.; Keyzers, R.A.; Prinsep, M.R. Marine natural products. Nat. Prod. Rep. 2014, 31, 160-258.

8. Gerwick, W.H.; Moore, B.S. Lessons from the past and charting the future of marine natural products drug discovery and chemical biology. Chem. Biol. 2012, 19, 85-98. [CrossRef]

9. Retter, A.; Nilsson, R.H.; Bourlat, S.J. Exploring the taxonomic composition of two fungal communities on the Swedish west coast through metabarcoding. Biodivers. Data J. 2019, 7, e35332. [CrossRef]

10. Fan, B.; Parrot, D.; Blümel, M.; Labes, A.; Tasdemir, D. Influence of OSMAC-based cultivation in metabolome and anticancer activity of fungi associated with the brown alga Fucus Vesiculosus. Mar. Drugs 2019, 17, 67. [CrossRef]

11. Furbino, L.E.; Pellizzari, F.M.; Neto, P.C.; Rosa, C.A.; Rosa, L.H. Isolation of fungi associated with macroalgae from maritime Antarctica and their production of agarolytic and carrageenolytic activities. Polar Biol. 2018, 41, 527-535. [CrossRef]

12. Panno, L.; Bruno, M.; Voyron, S.; Anastasi, A.; Gnavi, G.; Miserere, L.; Varese, G.C. Diversity, ecological role and potential biotechnological applications of marine fungi associated to the seagrass Posidonia Oceanica. New Biotechnol. 2013, 30, 685-694. [CrossRef]

13. Jones, E.B.G.; Sakayaroj, J.; Suetrong, S.; Somrithipol, S.; Pang, K.L. Classification of marine Ascomycota, anamorphic taxa and Basidiomycota. Fungal Divers. 2009, 35, 187.

14. Jones, E.B.G. Are there more marine fungi to be described? Bot. Mar. 2011, 54, 343-354. [CrossRef]

15. Marinefungi. Available online: https://www.marinefungi.org/ (accessed on 9 November 2021).

16. Hasan, S.; Ansari, M.I.; Ahmad, A.; Mishra, M. Major bioactive metabolites from marine fungi: A Review. Bioinformation 2015, 11, 176-181. [CrossRef]

17. Jayawardena, R.S.; Hyde, K.D.; Chen, Y.J.; Papp, V.; Palla, B.; Papp, D.; Bhunjun, C.S.; Hurdeal, V.G.; Senwanna, C.; Manawasinghe, I.S.; et al. One stop shop IV: Taxonomic update with molecular phylogeny for important phytopathogenic genera: 76-100 (2020). Fungal Divers. 2020, 103, 87-218. [CrossRef]

18. Bensch, K.; Braun, U.; Groenewald, J.Z.; Crous, P.W. The genus Cladosporium. Stud. Mycol. 2012, 72, 1-401. [CrossRef]

19. Levetin, E.; Dorsey, K. Contribution of leaf surface fungi to the air spora. Aerobiologia 2006, 22, 3-12. [CrossRef] 
20. Heuchert, B.; Braun, U.; ScHuBert, K. Morphotaxonomic revision of fungi-colous Cladosporium species (hyphomycetes). Schlechtendalia 2005, 13, 1-78.

21. Bensch, K.; Groenewald, J.Z.; Meijer, M.; Dijksterhuis, J.; Jurjević, Ž.; Andersen, B.; Houbraken, J.; Crous, P.W.; Samson, R.A. Cladosporium species in indoor environments. Stud. Mycol. 2018, 89, 177-301.

22. El-Dawy, E.G.A.E.M.; Gherbawy, Y.A.; Hussein, M.A. Morphological, molecular characterization, plant pathogenicity and biocontrol of Cladosporium complex groups associated with faba beans. Sci. Rep. 2021, 11, 14183. [CrossRef] [PubMed]

23. Denning, D.W.; O'Driscoll, B.R.; Hogaboam, C.M.; Bowyer, P.; Niven, R.M. The link between fungi and severe asthma: A summary of the evidence. Eur. Resp. J. 2006, 27, 615-626. [CrossRef] [PubMed]

24. Van Kan, J.A.; Van den Ackerveken, G.F.J.M.; De Wit, P.J.G.M. Cloning and characterization of cDNA of avirulence gene avr9 of the fungal pathogen Cladosporium fulvum, causal agent of tomato leaf mold. Mol. Plant Microbe Interact. 1991, 4, 52-59. [CrossRef] [PubMed]

25. Batra, N.; Kaur, H.; Mohindra, S.; Singh, S.; Shamanth, A.S.; Rudramurthy, S.M. Cladosporium sphaerospermum causing brain abscess, a saprophyte turning pathogen: Case and review of published reports. J. Mycol. Med. 2019, 29, 180-184. [CrossRef]

26. Sandoval-Denis, M.; Gené, J.; Sutton, D.A.; Wiederhold, N.P.; Cano-Lira, J.F.; Guarro, J. New species of Cladosporium associated with human and animal infections. Persoonia 2016, 36, 281-298. [CrossRef]

27. Simon-Nobbe, B.; Denk, U.; Pöll, V.; Rid, R.; Breitenbach, M. The spectrum of fungal allergy. Int. Arch. Allergy Immunol. 2008, 145, 58-86. [CrossRef]

28. De Hoog, G.S.; Guarro, J.; Gené, J.; Figueras, M.J. Atlas of Clinical Fungi, 2nd ed.; Centraalbureau voor Schimmelcultures (CBS): Utrecht, The Netherlands, 2000.

29. Hamayun, M.; Khan, S.A.; Ahmad, N.; Tang, D.S.; Kang, S.M.; Na, C.I.; Sohn, E.Y.; Hwang, Y.H.; Shin, D.H.; Lee, B.H.; et al. Cladosporium sphaerospermum as a new plant growth-promoting endophyte from the roots of Glycine max (L.) Merr. World J. Microbiol. Biotechnol. 2009, 25, 627-632. [CrossRef]

30. Sellart-Altisent, M.; Torres-Rodríguez, J.M.; Gómez de Ana, S.; Alvarado-Ramírez, E. Microbiota fúngica nasal en sujetos alérgicos y sanos [Nasal fungal microbiota in allergic and healthy subjects]. Rev. Iberoam. Micol. 2007, 24, 125-130. [CrossRef]

31. Sandoval-Denis, M.; Sutton, D.A.; Martin-Vicente, A.; Cano-Lira, J.F.; Wiederhold, N.; Guarro, J.; Gené, J. Cladosporium species recovered from clinical samples in the United States. J. Clin. Microbiol. 2015, 53, 2990-3000. [CrossRef]

32. Sun, J.Z.; Liu, X.Z.; McKenzie, E.H.C.; Jeewon, R.; Liu, J.K.; Zhang, X.L.; Zhao, Q.; Hyde, K.D. Fungicolous fungi: Terminology, diversity, distribution, evolution and species checklist. Fungal Divers. 2019, 95, 337-430. [CrossRef]

33. Torres, D.E.; Rojas-Martínez, R.I.; Zavaleta-Mejia, E.; Guevara-Fefer, P.; Márquez-Guzmán, G.J.; Perez-Martinez, C. Cladosporium cladosporioides and Cladosporium pseudocladosporioides as potential new fungal antagonists of Puccinia horiana Henn., the causal agent of Chrysanthemum white rust. PLoS ONE 2017, 12, e0170782. [CrossRef]

34. Jashni, M.K.; van der Burgt, A.; Battaglia, E.; Mehrabi, R.; Collemare, J.; de Wit, P.J. Transcriptome and proteome analyses of proteases in biotroph fungal pathogen Cladosporium fulvum. J. Plant Pathol. 2020, 102, 377-386. [CrossRef]

35. Ge, J.; Yin, Y.; Jiang, X.; Liu, W.; Yao, B.; Luo, H. Gene cloning and characterization of a novel glucose oxidase from Cladosporium tianshanense SL19. J. Agric. Sci. Technol. 2019, 12, 49-57.

36. Amin, M.; Zhang, X.Y.; Xu, X.Y.; Qi, S.H. New citrinin derivatives from the deep-sea-derived fungus Cladosporium sp. SCSIO z015. Nat. Prod. Res. 2020, 34, 1219-1226. [CrossRef]

37. Pan, F.; El-Kashef, D.H.; Kalscheuer, R.; Müller, W.E.G.; Lee, J.; Feldbrügge, M.; Mándi, A.; Kurtán, T.; Liu, Z.; Wu, W.; et al. New hybrid polyketides from the endophytic fungus Cladosporium sphaerospermum WBS017. Eur. J. Med. Chem. 2020, 191, 112159. [CrossRef]

38. Rischer, M.; Lee, S.R.; Eom, H.J.; Park, H.B.; Vollmers, J.; Kaster, A.-K.; Shin, Y.-H.; Oh, D.-C.; Kim, K.H.; Beemelmanns, C. Spirocyclic cladosporicin A and cladosporiumins I and J from a Hydractinia-associated Cladosporium sphaerospermum SW67. Org. Chem. Front. 2019, 6, 1084-1093. [CrossRef]

39. Zhang, F.Z.; Li, X.M.; Yang, S.Q.; Meng, L.H.; Wang, B.G. Thiocladospolides A-D, 12-membered macrolides from the mangrovederived endophytic fungus Cladosporium cladosporioides MA-299 and structure revision of pandangolide 3. J. Nat. Prod. 2019, 82, 1535-1541. [CrossRef]

40. Zhang, F.Z.; Li, X.M.; Li, X.; Yang, S.Q.; Meng, L.H.; Wang, B.G. Polyketides from the mangrove-derived endophytic fungus Cladosporium cladosporioides. Mar. Drugs 2019, 17, 296. [CrossRef]

41. Zhu, M.; Gao, H.; Wu, C.; Zhu, T.; Che, Q.; Gu, Q.; Guo, P.; Li, D. Lipid-lowering polyketides from a soft coral-derived fungus Cladosporium sp. TZP29. Bioorg. Med. Chem. Lett. 2015, 25, 3606-3609. [CrossRef]

42. Wu, G.; Sun, X.; Yu, G.; Wang, W.; Zhu, T.; Gu, Q.; Li, D. Cladosins A-E, hybrid polyketides from a deep-sea-derived fungus, Cladosporium sphaerospermum. J. Nat. Prod. 2014, 77, 270-275. [CrossRef]

43. Birolli, W.G.; Zanin, L.L.; Jimenez, D.E.Q.; Porto, A.L.M. Synthesis of Knoevenagel adducts under microwave irradiation and biocatalytic ene-reduction by the marine-derived fungus Cladosporium sp. CBMAI 1237 for the production of 2-cyano-3phenylpropanamide derivatives. Mar. Biotechnol. 2020, 22, 317-330. [CrossRef]

44. Birolli, W.G.; de Santos, A.D.; Alvarenga, N.; Garcia, A.C.F.S.; Romão, L.P.C.; Porto, A.L.M. Biodegradation of anthracene and several PAHs by the marine-derived fungus Cladosporium sp. CBMAI 1237. Mar. Pollut. Bull. 2018, 129, 525-533. [CrossRef]

45. Gil-Durán, C.; Ravanal, M.C.; Ubilla, P.; Vaca, I.; Chávez, R. Heterologous expression, purification and characterization of a highly thermolabile endoxylanase from the Antarctic fungus Cladosporium sp. Fungal Biol. 2018, 122, 875-882. [CrossRef] 
46. Taskin, M.; Ortucu, S.; Unver, Y.; Tasar, O.C.; Ozdemir, M.; Kaymak, H.C. Invertase production and molasses decolourization by cold-adapted filamentous fungus Cladosporium herbarum ER-25 in non-sterile molasses medium. Process Saf. Environ. Prot. 2016, 103, 136-143. [CrossRef]

47. Trivedi, N.; Reddy, C.; Radulovich, R.; Jha, B. Solid state fermentation (SSF)-derived cellulase for saccharification of the green seaweed Ulva for bioethanol production. Algal Res. 2015, 9, 48-54. [CrossRef]

48. Del-Cid, A.; Ubilla, P.; Ravanal, M.C.; Medina, E.; Vaca, I.; Levicán, G.; Eyzaguirre, J.; Chávez, R. Cold-active xylanase produced by fungi associated with Antarctic marine sponges. Appl. Biochem. Biotechnol. 2014, 172, 524-532. [CrossRef]

49. Bastos, S.C.; Pimenta, C.J.; Dias, D.R.; Chalfoun, S.M.; Ange'lico, C.L.; Tavares, L.S. Pectinases from a new strain of Cladosporium cladosporioides (Fres.) De Vries isolated from coffee bean. World J. Agric. Sci. 2013, 9, 167-172.

50. Bonugli-Santos, R.C.; Durrant, L.R.; da Silva, M.; Sette, L.D. Production of laccase, manganese peroxidase and lignin peroxidase by Brazilian marine-derived fungi. Enzyme Microb. Technol. 2010, 46, 32-37. [CrossRef]

51. Duarte, A.W.F.; Dayo-Owoyemi, I.; Nobre, F.S.; Pagnocca, F.C.; Chaud, L.C.S.; Pessoa, A.; Felipe, M.G.; Sette, L.D. Taxonomic assessment and enzymes production by yeasts isolated from marine and terrestrial Antarctic samples. Extremophiles 2013, 17, 1023-1035. [CrossRef]

52. Ball, A.; Truskewycz, A. Polyaromatic hydrocarbon exposure: An ecological impact ambiguity. Environ. Sci. Pollut. Res. 2013, 20, 4311-4326. [CrossRef]

53. Lee, S.R.; Lee, D.; Eom, H.J.; Rischer, M.; Ko, Y.-J.; Kang, K.S.; Kim, C.S.; Beemelmanns, C.; Kim, K.H. Hybrid polyketides from a Hydractinia-associated Cladosporium sphaerospermum SW67 and their putative biosynthetic origin. Mar. Drugs 2019, 17, 606. [CrossRef] [PubMed]

54. Yu, G.H.; Wu, G.W.; Zhu, T.J.; Gu, Q.Q.; Li, D.H. Cladosins F and G, two new hybrid polyketides from the deep-sea-derived Cladosporium sphaerospermum 2005-01-E3. J. Asian Nat. Prod. Res. 2015, 17, 120-124. [CrossRef] [PubMed]

55. Zhang, Z.; He, X.; Wu, G.; Liu, C.; Lu, C.; Gu, Q.; Che, Q.; Zhu, T.; Zhang, G.; Li, D. Aniline-tetramic acids from the deep-seaderived fungus Cladosporium sphaerospermum L3P3 cultured with the HDAC inhibitor SAHA. J. Nat. Prod. 2018, 81, 1651-1657. [CrossRef] [PubMed]

56. Zhu, G.; Kong, F.; Wang, Y.; Fu, P.; Zhum, W. Cladodionen, a cytotoxic hybrid polyketide from the marine-derived Cladosporium sp. OUCMDZ-1635. Mar. Drugs 2018, 16, 71. [CrossRef]

57. Liang, X.; Huang, Z.H.; Ma, X.; Qi, S.H. Unstable tetramic acid derivatives from the deep-sea-derived fungus Cladosporium sphaerospermum EIODSF 008. Mar. Drugs 2018, 16, 448. [CrossRef]

58. Huang, Z.-H.; Nong, X.-H.; Liang, X.; Qi, S.-H. New tetramic acid derivatives from the deep-sea-derived fungus Cladosporium sp. SCSIO z0025. Tetrahedron 2018, 74, 2620-2626. [CrossRef]

59. Ding, L.; Qin, S.; Li, F.; Chi, X.; Laatsch, H. Isolation, antimicrobial activity, and metabolites of fungus Cladosporium sp. associated with red alga Porphyra yezoensis. Curr. Microbiol. 2008, 56, 229-235. [CrossRef]

60. Qi, S.-H.; Xu, Y.; Xiong, H.-R.; Qian, P.-Y.; Zhang, S. Antifouling and antibacterial compounds from a marine fungus Cladosporium sp. F14. World J. Microbiol. Biotechnol. 2009, 25, 399-406. [CrossRef]

61. Pang, X.; Lin, X.; Wang, J.; Liang, R.; Tian, Y.; Salendra, L.; Luo, X.; Zhou, X.; Yang, B.; Tu, Z.; et al. Three new highly oxygenated sterols and one new dihydroisocoumarin from the marine sponge-derived fungus Cladosporium sp. SCSIO41007. Steroids 2018, 129, 41-46. [CrossRef]

62. Gu, B.; Zhang, Y.; Ding, L.; He, S.; Wu, B.; Dong, J.; Zhu, P.; Chen, J.; Zhang, J.; Yan, X. Preparative separation of sulfur-containing diketopiperazines from marine fungus Cladosporium sp. using high-speed counter-current chromatography in stepwise elution mode. Mar. Drugs 2015, 13, 354-365. [CrossRef]

63. Manríquez, V.; Galdámeza, A.; Veliz, B.; Rovirosa, J.; Díaz-Marrero, A.R.; Cueto, M.; Darias, J.; Martínez, C.; San-Martín, A. $\mathrm{N}$-methyl-1H-indole-2-carboxamide from the marine fungus Cladosporium cladosporioides. J. Chil. Chem. Soc. 2009, 54, 314-316. [CrossRef]

64. Peng, J.; Lin, T.; Wang, W.; Xin, Z.; Zhu, T.; Gu, Q.; Li, D. Antiviral alkaloids produced by the mangrove-derived fungus Cladosporium sp. PJX-41. J. Nat. Prod. 2013, 76, 1133-1140. [CrossRef]

65. Yang, G.; Nenkep, V.N.; Siwe, X.N.; Leutou, A.S.; Feng, Z.H.; Zhang, D.; Choi, H.D.; Kang, J.S.; Son, B.W. An acetophenone derivative, clavatol, and a benzodiazepine alkaloid, circumdatin A, from the marine-derived fungus Cladosporium. Nat. Prod. Sci. 2009, 15, 130-133.

66. Kyeremeh, K.; Owusu, K.B.-A.; Ofosuhene, M.; Ohashi, M.; Agyapong, J.; Camas, A.S.; Camas, M. Anti-proliferative and antiplasmodia activity of quinolactacin A2, citrinadin A and butrecitrinadin co-isolated from a Ghanaian mangrove endophytic fungus Cladosporium oxysporum strain BRS2A-AR2F. J. Chem. Appl. 2017, 3, 12.

67. Wang, P.; Cui, Y.; Cai, C.; Chen, H.; Dai, Y.; Chen, P.; Kong, F.; Yuan, J.; Song, X.; Mei, W.; et al. Two new succinimide derivatives cladosporitins A and B from the mangrove-derived fungus Cladosporium sp. HNWSW-1. Mar. Drugs 2019, 17, 4. [CrossRef]

68. Rotinsulu, H.; Yamazaki, H.; Sugai, S.; Iwakura, N.; Wewengkang, D.S.; Sumilat, D.A.; Namikoshi, M. Cladosporamide A, a new protein tyrosine phosphatase 1B inhibitor, produced by an Indonesian marine sponge-derived Cladosporium sp. J. Nat. Med. 2018, 72, 779-783. [CrossRef]

69. Cao, F.; Yang, Q.; Shao, C.L.; Kong, C.J.; Zheng, J.J.; Liu, Y.F.; Wang, C.Y. Bioactive 7-oxabicyclic[6.3.0]lactam and 12-membered macrolides from a gorgonian-derived Cladosporium sp. fungus. Mar. Drugs 2015, 13, 4171-4178. [CrossRef] 
70. Huang, C.; Chen, T.; Yan, Z.; Guo, H.; Hou, X.; Jiang, L.; Long, Y. Thiocladospolide E and cladospamide A, novel 12-membered macrolide and macrolide lactam from mangrove endophytic fungus Cladosporium sp. SCNU-F0001. Fitoterapia 2019, $137,104246$. [CrossRef]

71. Bai, M.; Zheng, C.J.; Tang, D.Q.; Zhang, F.; Wang, H.Y.; Chen, G.Y. Two new secondary metabolites from a mangrove-derived fungus Cladosporium sp. JS1-2. J. Antibiot. 2019, 72, 779-782. [CrossRef]

72. Zhang, H.; Tomoda, H.; Tabata, N.; Miura, H.; Namikoshi, M.; Yamaguchi, Y.; Masuma, R.; Omura, S. Cladospolide D, a new 12-membered macrolide antibiotic produced by Cladosporium sp. FT-0012. J. Antibiot. 2001, 54, 635-641. [CrossRef]

73. Wuringege Guo, Z.K.; Wei, W.; Jiao, R.H.; Yan, T.; Zang, L.Y.; Jiang, R.; Tan, R.X.; Ge, H.M. Polyketides from the plant endophytic fungus Cladosporium sp. IFB3lp-2. J. Asian Nat. Prod. Res. 2013, 15, 928-933. [CrossRef]

74. Jadulco, R.; Proksch, P.; Wray, V.; Sudarsono Berg, A.; Gräfe, U. New macrolides and furan carboxylic acid derivative from the sponge-derived fungus Cladosporium herbarum. J. Nat. Prod. 2001, 64, 527-530. [CrossRef]

75. He, Z.H.; Zhang, G.; Yan, Q.X.; Zou, Z.B.; Xiao, H.X.; Xie, C.L.; Tang, X.X.; Luo, L.Z.; Yang, X.W. Cladosporactone A, a unique polyketide with 7-methylisochromen-3-one skeleton from the deep-sea-derived fungus Cladosporium cladosporioides. Chem. Biodivers. 2020, 17, e2000158. [CrossRef]

76. Gao, C.H.; Nong, X.H.; Qi, S.H.; Luo, X.M.; Zhang, S.; Xiong, H.R. A new nine-membdered lactone from a marine fungus Cladosporium sp. F14. Chin. Chem. Lett. 2010, 21, 1355-1357. [CrossRef]

77. Gesner, S.; Cohen, N.; Ilan, M.; Yarden, O.; Carmeli, S. Pandangolide 1a, a metabolite of the sponge-associated fungus Cladosporium sp., and the absolute stereochemistry of pandangolide 1 and iso-cladospolide B. J. Nat. Prod. 2005, 68, 1350-1353. [CrossRef]

78. Wang, W.; Feng, H.; Sun, C.; Che, Q.; Zhang, G.; Zhu, T.; Li, D. Thiocladospolides F-J, antibacterial sulfur containing 12membered macrolides from the mangrove endophytic fungus Cladosporium oxysporum HDN13-314. Phytochemistry 2020, 178, 112462. [CrossRef]

79. Zhang, F.Z.; Li, X.M.; Meng, L.H.; Wang, B.G. Cladocladosin A, an unusual macrolide with bicyclo 5/9 ring system, and two thiomacrolides from the marine mangrove-derived endophytic fungus, Cladosporium cladosporioides MA-299. Bioorg. Chem. 2020, 101, 103950. [CrossRef]

80. Shigemori, H.; Kasai, Y.; Komatsu, K.; Tsuda, M.; Mikami, Y.; Kobayashi, J. Sporiolides A and B, new cytotoxic twelve-membered macrolides from a marine-derived fungus Cladosporium pecies. Mar. Drugs 2004, 2, 164-169. [CrossRef]

81. Peng, X.; Wang, Y.; Zhu, G.; Zhu, W. Fatty acid derivatives from the halotolerant fungus Cladosporium cladosporioides. Magn. Reson. Chem. 2018, 56, 18-24. [CrossRef]

82. Ai, W.; Lin, X.; Wang, Z.; Lu, X.; Mangaladoss, F.; Yang, X.; Zhou, X.; Tu, Z.; Liu, Y. Cladosporone A, a new dimeric tetralone from fungus Cladosporium sp. KcFL6' derived of mangrove plant Kandelia candel. J. Antibiot. 2015, 68, 213-215. [CrossRef]

83. Zhang, Z.; He, X.; Liu, C.; Che, Q.; Zhu, T.; Gu, Q.; Li, D. Clindanones A and B and cladosporols F and G, polyketides from the deep-sea derived fungus Cladosporium cladosporioides HDN14-342. RSC Adv. 2016, 6, 76498-76504. [CrossRef]

84. Li, H.L.; Li, X.M.; Mándi, A.; Antus, S.; Li, X.; Zhang, P.; Liu, Y.; Kurtán, T.; Wang, B.G. Characterization of cladosporols from the marine algal-derived endophytic fungus Cladosporium cladosporioides en-399 and configurational revision of the previously reported cladosporol derivatives. J. Org. Chem. 2017, 82, 9946-9954. [CrossRef] [PubMed]

85. Zhang, F.; Zhou, L.; Kong, F.; Ma, Q.; Xie, Q.; Li, J.; Dai, H.; Guo, L.; Zhao, Y. Altertoxins with quorum sensing inhibitory activities from the marine-derived fungus Cladosporium sp. KFD33. Mar. Drugs 2020, 18, 67. [CrossRef] [PubMed]

86. Zhen, F.; Zhang-hua, S.; Yu-chan, C.; Hao-hua, L.; Wei-min, Z. Cladosperanol A, a new dimeric tetralone from marine-derived fungus cladosporium perangustum. Nat. Prod. Res. Dev. 2016, 28, 486-489.

87. Fan, Z.; Sun, Z.H.; Liu, H.X.; Chen, Y.C.; Li, H.H.; Zhang, W.M. Perangustols A and B, a pair of new azaphilone epimers from a marine sediment-derived fungus Cladosporium perangustm FS62. J. Asian Nat. Prod. Res. 2016, 18, 1024-1029. [CrossRef]

88. Wu, J.T.; Zheng, C.J.; Zhang, B.; Zhou, X.M.; Zhou, Q.; Chen, G.Y.; Zeng, Z.E.; Xie, J.L.; Han, C.R.; Lyu, J.X. Two new secondary metabolites from a mangrove-derived fungus Cladosporium sp. JJM22. Nat. Prod. Res. 2019, 33, 34-40. [CrossRef]

89. Fan, C.; Zhou, G.; Wang, W.; Zhang, G.; Zhu, T.; Che, Q.; Li, D. Tetralone derivatives from a deep-sea derived fungus Cladosporium sp. HDN17-58. Nat. Prod. Commun. 2021, 16, 1934578X211008322. [CrossRef]

90. Ma, R.-Z.; Zheng, C.-J.; Zhang, B.; Yang, J.-Y.; Zhou, X.-M.; Song, X.-M. Two New naphthalene-chroman coupled derivatives from the mangrove-derived fungus Cladosporium sp. JJM22. Phytochem. Lett. 2021, 43, 114-116. [CrossRef]

91. Li, Z.; Yang, J.Y.; Caj, J.; Ouyang, Z.J.; Zhou, C.H.; Chen, G.Y.; Zhou, X.M. Study on secondary metabolites of endophytic fungus Cladosporium sp. JJM22 hosted in Ceriops tagal. Zhongguo Zhong Yao Za Zhi 2021, 46, 2079-2083.

92. Wang, C.N.; Lu, H.M.; Gao, C.H.; Guo, L.; Zhan, Z.Y.; Wang, J.J.; Liu, Y.H.; Xiang, S.T.; Wang, J.; Luo, X.W. Cytotoxic benzopyranone and xanthone derivatives from a coral symbiotic fungus Cladosporium halotolerans GXIMD 02502. Nat. Prod. Res. 2020, 1-8. [CrossRef]

93. Silber, J.; Ohlendorf, B.; Labes, A.; Wenzel-Storjohann, A.; Näther, C.; Imhoff, J.F. Malettinin E, an antibacterial and antifungal tropolone produced by a marine Cladosporium strain. Front. Mar. Sci. 2014, 1, 35. [CrossRef]

94. Liu, Y.; Kurtán, T.; Yun Wang, C.; Han Lin, W.; Orfali, R.; Müller, W.E.; Daletos, G.; Proksch, P. Cladosporinone, a new viriditoxin derivative from the hypersaline lake derived fungus Cladosporium cladosporioides. J. Antibiot. 2016, 69, 702-706. [CrossRef]

95. Wang, L.; Han, X.; Zhu, G.; Wang, Y.; Chairoungdua, A.; Piyachaturawat, P.; Zhum, W. Polyketides from the endophytic fungus Cladosporium sp. isolated from the mangrove plant Excoecaria agallocha. Front. Chem. 2018, 6, 344. [CrossRef] 
96. Jadulco, R.; Brauers, G.; Edrada, R.A.; Ebel, R.; Wray, V.; Sudarsono, S.; Proksch, P. New metabolites from sponge-derived fungi Curvularia lunata and Cladosporium herbarum. J. Nat. Prod. 2002, 65, 730-733. [CrossRef]

97. Zhang, B.; Wu, J.T.; Zheng, C.J.; Zhou, X.M.; Yu, Z.X.; Li, W.S.; Chen, G.Y.; Zhu, G.Y. Bioactive cyclohexene derivatives from a mangrove-derived fungus Cladosporium sp. JJM22. Fitoterapia 2021, 149, 104823. [CrossRef]

98. San-Martin, A.; Painemal, K.; Diaz, Y.; Martinez, C.; Rovirosa, J. Metabolites from the marine fungus Cladosporium cladosporioides. J. Argent. Chem. Soc. 2005, 93, 247-251.

99. Kuznetsova, T.A.; Afiyatullov, S.A.; Denisenko, V.A.; Pivkin, M.V.; Elyakov, G.B. Sterols from a marine isolate of the fungus Cladosporium sphaerospermum Penz. Biochem. Syst. Ecol. 1998, 26, 365-366. [CrossRef]

100. Yu, M.L.; Guan, F.F.; Cao, F.; Jia, Y.L.; Wang, C.Y. A new antiviral pregnane from a gorgonian-derived Cladosporium sp. fungus. Nat. Prod. Res. 2018, 32, 1260-1266. [CrossRef]

101. Lee, S.R.; Kang, H.; Yoo, M.J.; Yu, J.S.; Lee, S.; Yi, S.A.; Beemelmanns, C.; Lee, J.; Kim, K.H. Anti-adipogenic pregnane steroid from a Hydractinia-associated fungus, Cladosporium sphaerospermum SW67. Nat. Prod. Sci. 2020, 26, $230-235$.

102. Gallo, M.L.; Seldes, A.M.; Cabrera, G.M. Antibiotic long-chain and $\alpha, \beta$-unsaturated aldehydes from the culture of the marine fungus Cladosporium sp. Biochem. Syst. Ecol. 2004, 32, 545-551. [CrossRef]

103. AlMatar, M.; Makky, E.A. Cladosporium cladosporioides from the perspectives of medical and biotechnological approaches. 3 Biotech 2016, 6, 4. [CrossRef]

104. Armisén, R.; Galatas, F.; Hispanagar, A.S. Agar. In Handbook of Hydrocolloids; Phillips, G.O., Williams, P.A., Eds.; Woodhead Publishing: Cambridge, UK, 2000; pp. 21-40.

105. Wang, J.; Jiang, X.; Mou, H.; Guan, H. Anti-oxidation of agar oligosaccharides produced by agarase from a marine bacterium. J. Appl. Phycol. 2004, 16, 333-340. [CrossRef]

106. Necas, J.; Bartosikova, L. Carrageenan: A review. Vet. Med. 2013, 58, 187-205. [CrossRef]

107. Ibrahim, S.R.M.; Mohamed, S.G.A.; Altyar, A.E.; Mohamed, G.A. Natural products of the fungal genus Humicola: Diversity, biological activity, and industrial importance. Curr. Microbiol. 2021, 78, 2488-2509. [CrossRef]

108. Ibrahim, S.R.M.; Mohamed, S.G.A.; Sindi, I.A.; Mohamed, G.A. Biologically active secondary metabolites and biotechnological applications of species of the family Chaetomiaceae (Sordariales): An updated review from 2016 to 2021. Mycol. Prog. 2021, 20, 595-639. [CrossRef]

109. Ibrahim, S.R.M.; Mohamed, S.G.A.; Altyar, A.E.; Mohamed, G.A. Genus Thielavia: Phytochemicals, industrial importance and biological relevance. Nat. Prod. Res. 2021, 1-16. [CrossRef]

110. Collins, T.; Gerday, C.; Feller, G. Xylanases, xylanase families and extremophilic xylanases. FEMS Microb. Rev. 2005, 29 , 3-23. [CrossRef] [PubMed]

111. D'Souza, D.T.; Tiwari, R.; Sah, A.K.; Raghukumara, C. Enhanced production of laccase by a marine fungus during treatment of colored effluents and synthetic dyes. Enzym. Microb. Technol. 2006, 38, 504-511. [CrossRef]

112. Kuwahara, M.; Glenn, J.K.; Morgan, M.A.; Gold, M.H. Separation and characterization of two extracellular $\mathrm{H}_{2} \mathrm{O}_{2}$ dependent oxidases from ligninolytic cultures of Phanerochaete chrysosporium. FEBS Lett. 1984, 169, 247-250. [CrossRef]

113. Raghukumar, C.; Muraleedharan, U.; Gaud, V.R.; Mishra, R. Simultaneous detoxification and decolorization of molasses spent wash by the immobilized white-rot fungus Flavodon flavusisolated from a marine habitat. Enzym. Microb. Technol. 2004, 35, 197-202. [CrossRef]

114. Da Silva, M.; Passarini, M.R.Z.; Bonugli, R.C.; Sette, L.D. Cnidarian-derived filamentous fungi from Brazil: Isolation, characterisation and RBBR decolourisation screening. Environ. Technol. 2008, 29, 1331-1339. [CrossRef]

115. Panno, L.; Voyron, S.; Anastasi, A.; Mussat Sarto, R.; Varese, G.C. Biodiversity of marine fungi associated with the seagrass Posidonia oceanica: An ecological and biotechnological perspective. Biol. Mar. Mediterr. 2011, 18, 85-88.

116. Taskin, M.; Esim, N.; Genisel, M.; Ortucu, S.; Hasenekoglu, I.; Canli, O.; Erdal, S. Enhancement of invertase production by Aspergillus niger OZ-3 using low-intensity static magnetic fields. Prep. Biochem. Biotechnol. 2013, 43, 177-188. [CrossRef]

117. Veana, F.; Martínez-Hernández, J.L.; Aguilar, N.; Rodríguez-Herrera, R.; Michelena, G. Utilization of molasses and sugar cane bagasse for production of fungal invertase in solid state fermentation using Aspergillus niger GH1. Braz. J. Microbiol. 2014, 5, 373-377. [CrossRef]

118. Yadav, S.; Chandra, R. Biodegradation of organic compounds of molasses melanoidin (MM) from biomethanated distillery spent wash (BMDS) during the decolourisation by a potential bacterial consortium. Biodegradation 2012, 23, 609-620. [CrossRef]

119. Gavrilescua, M.; Chisti, Y. Biotechnology-a sustainable alternative for chemical industry. Biotechnol. Adv. 2005, 23, 471-499. [CrossRef]

120. Winkler, C.K.; Faber, K.; Hall, M. Biocatalytic reduction of activated C=C-bonds and beyond: Emerging trends. Curr. Opin. Chem. Biol. 2018, 43, 97-105. [CrossRef]

121. Garzon-Posse, F.; Becerra-Figueroa, L.; Hernandez-Arias, J.; Gamba-Sanchez, D. Whole cells as biocatalysts in organic transformations. Molecules 2018, 23, 1265. [CrossRef]

122. Khare, R.; Pandey, J.; Smriti, R.R. The importance and applications of Knoevenagel reaction (brief review). Orient. J. Chem. 2019, 35, 423-429. [CrossRef]

123. Rocha, L.C.; Ferreira, H.V.; Luiz, R.F.; Sette, L.D.; Porto, A.L. Stereoselective bioreduction of 1-(4-methoxyphenyl) ethanone by whole cells of marine-derived fungi. Mar. Biotechnol. 2012, 14, 358-362. [CrossRef] 
124. Rocha, L.C.; Seleghim, M.H.R.; Comasseto, J.V.; Sette, L.D.; Porto, A.L.M. Stereoselective bioreduction of alpha-azido ketones by whole cells of marine-derived fungi. Mar. Biotechnol. 2015, 17, 736-742. [CrossRef] [PubMed]

125. Athanasellis, G.; Igglessi-Markopoulou, O.; Markopoulos, J. Tetramic and tetronic acids as scaffolds in bioinorganic and bioorganic chemistry. Bioinorg. Chem. Appl. 2010, 2010, 315056. [CrossRef] [PubMed]

126. Royles, B.J.L. Naturally occurring tetramic acids: Structure, isolation, and synthesis. Chem. Rev. 1995, 95, 1981-2001. [CrossRef]

127. Mo, X.; Li, Q.; Ju, J. Naturally occurring tetramic acid products: Isolation, structure elucidation and biological activity. RSC Adv. 2014, 4, 50566-50593. [CrossRef]

128. Jiang, M.; Chen, S.; Li, J.; Liu, L. The Biological and Chemical Diversity of Tetramic Acid Compounds from Marine-Derived Microorganisms. Mar. Drugs 2020, 18, 114. [CrossRef]

129. Gomes, N.G.M.; Pereira, R.B.; Andrade, P.B.; Valentao, P. Double the chemistry, double the fun: Structural diversity and biological activity of marine-derived diketopiperazine dimers. Mar. Drugs 2019, 17, 551. [CrossRef]

130. Huang, R.M.; Yi, X.X.; Zhou, Y.Y.; Su, X.D.; Peng, Y.; Gao, C.H. An update on 2,5-diketopiperazines from marine organisms. Mar. Drugs 2014, 12, 6213-6235. [CrossRef]

131. Borgman, P.; Lopez, R.D.; Lane, A.L. The expanding spectrum of diketopiperazine natural product biosynthetic pathways containing cyclodipeptide synthases. Org. Biomol. Chem. 2019, 17, 2305-2314. [CrossRef]

132. Song, Z.; Hou, Y.; Yang, Q.; Li, X.; Wu, S. Structures and Biological Activities of Diketopiperazines from Marine Organisms: A Review. Mar. Drugs 2021, 19, 403. [CrossRef]

133. Urban, S.; Hickford, S.J.H.; Blunt, J.W.; Munro, M.H.G.; Yue, Y.F.; Chang, Q.H.; Zhu, H.J.; Cao, F. Bioactive marine alkaloids. Curr. Org. Chem. 2000, 4, 765-807. [CrossRef]

134. Meng, Z.H.; Sun, T.T.; Zhao, G.Z.; Yue, Y.-F.; Chang, Q.-H.; Zhu, H.-J.; Cao, F. Marine-derived fungi as a source of bioactive indole alkaloids with diversified structures. Mar. Life Sci. Technol. 2021, 3, 44-61. [CrossRef]

135. Islam, M.T.; Mubarak, M.S. Pyrrolidine alkaloids and their promises in pharmacotherapy. Adv. Tradit. Med. 2020, $20,13-22$. [CrossRef]

136. Netz, N.; Opatz, T. Marine indole alkaloids. Mar. Drugs 2015, 13, 4814-4914. [CrossRef]

137. Woodward, R.B. Struktur und biogenese der makrolide. Angew. Chem. 1957, 69, 50-58. [CrossRef]

138. Janas, A.; Przybylski, P. 14- and 15-membered lactone macrolides and their analogues and hybrids: Structure, molecular mechanism of action and biological activity. Eur. J. Med. Chem. 2019, 182, 111662. [CrossRef]

139. Zhang, H.; Zou, J.; Yan, X.; Chen, J.; Cao, X.; Wu, J.; Liu, Y.; Wang, T. Marine-Derived Macrolides 1990-2020: An Overview of Chemical and Biological Diversity. Mar. Drugs 2021, 19, 180. [CrossRef]

140. Ray Choudhury, A.; Mukherjee, S. Deconjugated butenolide: A versatile building block for asymmetric catalysis. Chem. Soc. Rev. 2020, 49, 6755-6788. [CrossRef]

141. Kumar, A.; Singh, V.; Ghosh, S. Butenolide: A Novel Synthesis and Biological Activities, 1st ed.; LAP Lambert Academic Publishing: Saarbrücken, Germany, 2012.

142. Gibson, M.Z.; Nguyen, M.A.; Zingales, S.K. Design, synthesis, and evaluation of (2-(pyridinyl)methylene)-1-tetralone chalcones for anticancer and antimicrobial activity. Med. Chem. 2018, 14, 333-343. [CrossRef]

143. Gauni, B.; Mehariya, K.; Shah, A.; Duggirala, S.M. Tetralone scaffolds and their potential therapeutic applications. Lett. Drug Des. Disc. 2021, 18, 222-238. [CrossRef]

144. Zurlo, D.; Leone, C.; Assante, G.; Salzano, S.; Renzone, G.; Scaloni, A.; Foresta, C.; Colantuoni, V.; Lupo, A. Cladosporol a stimulates G1-phase arrest of the cell cycle by up-regulation of p21 waf1/cip1 expression in human colon carcinoma HT-29 cells. Mol. Carcinog. 2013, 52, 1-17. [CrossRef]

145. Zurlo, D.; Assante, G.; Moricca, S.; Colantuoni, V.; Lupo, A. Cladosporol A, a new peroxisome proliferator-activated receptor $\gamma$ $(\mathrm{PPAR} \gamma)$ ligand, inhibits colorectal cancer cells proliferation through $\beta$-catenin/TCF pathway inactivation. Biochim. Biophys. Acta 2014, 1840, 2361-2372. [CrossRef]

146. Mulrooey, C.A.; O’Brien, E.M.; Morgan, B.J.; Kozlowski, M.C. Perylenequinones: Isolation, synthesis, and biological activity. Eur. J. Org. Chem. 2012, 2012, 3887-3904. [CrossRef]

147. Stack, M.E.; Prival, M.J. Mutagenicity of the Alternaria metabolites altertoxins I., II, and III. Appl. Environ. Microbiol. 1986, 52, 718-722. [CrossRef]

148. Ibrahim, S.R.M.; Mohamed, G.A. Naturally occurring naphthalenes: Chemistry, biosynthesis, structural elucidation, and biological activities. Phytochem. Rev. 2016, 15, 279-295. [CrossRef]

149. Vieira, L.; Kijjoa, A. Naturally-occurring xanthones: Recent developments. Curr. Med. Chem. 2005, 12, 2413-2446. [CrossRef]

150. Ibrahim, S.R.M.; Abdallah, H.M.; El-Halawany, A.M.; Radwan, M.F.; Shehata, I.A.; Al-Harshany, E.M.; Zayed, M.F.; Mohamed, G.A. Garcixanthones B and C, new xanthones from the pericarps of Garcinia mangostana and their cytotoxic activity. Phytochem. Lett. 2018, 25, 12-16. [CrossRef]

151. Mohamed, G.A.; Al-Abd, A.M.; El-Halawany, A.M.; Abdallah, H.M.; Ibrahim, S.R.M. New xanthones and cytotoxic constituents from Garcinia mangostana fruit hulls against human hepatocellular, breast, and colorectal cancer cell lines. J. Ethnopharmacol. 2017, 198, 302-312. [CrossRef]

152. Guo, H.; Roman, D.; Beemelmanns, C. Tropolone natural products. Nat. Prod. Rep. 2019, 36, 1137-1155. [CrossRef]

153. Choque, E.; El Rayess, Y.; Raynal, J.; Mathieu, F. Fungal naphtho- $\gamma$-pyrones-Secondary metabolites of industrial interest. Appl. Microbiol. Biotechnol. 2015, 99, 1081-1096. [CrossRef] [PubMed] 
154. Suzuki, K.; Nozawa, K.; Nakajima, S.; Kawai, K. Structure revision of mycotoxin, viriditoxin, and its derivatives. Chem. Pharm. Bull. 1990, 38, 3180-3181. [CrossRef]

155. De Rosa, S.; De Giulio, A.; Iodice, C.; Alcaraz, M.J.; Paya, M. Long-chain aldehydes from the red alga, Corallina Mediterr. Phytochemistry 1995, 40, 995-996. [CrossRef]

156. Suzuki, M.; Kurosawa, E.; Kurata, K. (E)-2-Tridecyl-2-heptadecenal, an unusual metabolite from the red alga Laurencia sp. Bull. Chem. Soc. Jpn. 1987, 60, 3793-3794. [CrossRef]

157. Wulandari, A.P.; Examinati, R.R.I.N.; Madihah; Huspa, D.H.P.; Andayaningsih, P.; Andayaningsih, P. Cytotoxicity of metabolites produced by endophytic fungus Cladosporium sp. isolated from marine macroalgae on in-vitro MCF-7, HeLa, and DU-145 cell lines. Int. J. Pharm. Pharm. Sci. 2018, 10, 72-76. [CrossRef]

158. Hulikere, M.M.; Joshi, C.G.; Ananda, D.; Poyya, J.; Nivya, T. Antiangiogenic, wound healing and antioxidant activity of Cladosporium cladosporioides (Endophytic Fungus) isolated from seaweed (Sargassum wightii). Mycology 2016, 7, 203-211. [CrossRef]

159. Xiong, H.; Qi, S.; Xu, Y.; Miao, L.; Qian, P.Y. Antibiotic and antifouling compound production by the marine-derived fungus Cladosporium sp. F14. J. Hydro-Environ. Res. 2009, 2, 264-270. [CrossRef]

160. Hulikere, M.M.; Joshi, C.G.; Danagoudar, A.; Poyya, J.; Kudva, A.K.; Dhananjaya, B.L. Biogenic synthesis of gold nanoparticles by marine endophytic fungus Cladosporium cladosporioides isolated from seaweed and evaluation of their antioxidant and antimicrobial properties. Process Biochem. 2017, 63, 137-144.

161. Ameen, F.; Al-Homaidan, A.A.; Al-Sabr, A.; Almansob, A.; AlNAdhari, S. Anti-oxidant, anti-fungal and cytotoxic effects of silver nanoparticles synthesized using marine fungus Cladosporium halotolerans. Appl. Nanosci. 2021. [CrossRef]

162. Arnone, A.; Assante, G.; Modugno, V.D.; Merlini, L.; Nasini, G. Perylenequinones from cucumber seedlings infected with Cladosporium cucumerinum. Phytochemisry 1988, 27, 1675-1678. [CrossRef]

163. Arnone, A.; Camarda, L.; Nasini, G.; Merlini, L. Secondary mould metabolites. Part 13. Fungal perylenequinones: Phleichrome, isophleichrome, and their endoperoxides. J. Chem. Soc. Perkin Trans. 1 1985, 1387-1392. [CrossRef]

164. Arone, A.; Assante, G.; Merlini, L.; Nasini, G. Structure and stereochemistry of cladochrome D and E, novel perylenequinone pigment from Cladosporium cladosporioides. Gazetta Chem. Ital. 1989, 119, 557-559.

165. Mino, Y.; Idonuma, T.; Sakai, R. Effect of Phleichrome Produced by the Timothy Leaf Spot Fungus, Cladosporium phlei on the Invertases from the Host Leaves. Ann. Phytopathol. Soc. Jpn. 1979, 45, 463-467. [CrossRef]

166. Sassa, T.; Negoro, T.; Ueki, H. Production and Characterization of a new fungal metabolite, cotylenol. Agric. Biol. Chem. 1972, 36, 2281-2285. [CrossRef]

167. Sassa, T.; Ooi, T.; Nukina, M.; Ikeda, M.; Kato, N. Structural confirmation of cotylenin A, a novel fusicoccane-diterpene glycoside with potent plant growth-regulating activity from Cladosporium fungus sp. 501-7W. Biosci. Biotechnol. Biochem. 1998, 62, 1815-1818. [CrossRef]

168. Sassa, T.; Togashi, M.; Kitaguchi, T. The structures of cotylenins A., B., C., D and E. Agric. Biol. Chem. 1975, $39,1735-1744$. [CrossRef]

169. Sassa, T. Cotylenins, ${ }^{13}$ C NMR of cotylenins. Agric. Biol. Chem. 1979, 43, 385-387.

170. Sassa, T. Cotylenins, leaf growth substances produced by a fungus. Part, I. Isolation and characterization of cotylenins A and B. Agric. Biol. Chem. 1971, 35, 1415-1418.

171. Baral, B.; Akhgari, A.; Metsä-Ketelä, M. Activation of microbial secondary metabolic pathways: Avenues and challenges. Synth. Syst. Biotechnol. 2018, 3, 163-178. [CrossRef]

172. Reich, M.; Labes, A. How to boost marine fungal research: A first step towards a multidisciplinary approach by combining molecular fungal ecology and natural products chemistry. Mar. Genom. 2017, 36, 57-75. [CrossRef]

173. Scharf, D.H.; Brakhage, A.A. Engineering fungal secondary metabolism: A roadmap to novel compounds. J. Biotechnol. 2013, 163, 179-183. [CrossRef]

174. Reen, F.J.; Romano, S.; Dobson, A.D.W.; O'Gara, F. The sound of silence: Activating silent biosynthetic gene clusters in marine microorganisms. Mar. Drugs 2015, 13, 4754-4783. [CrossRef]

175. Bode, H.B.; Bethe, B.; Höfs, R.; Zeeck, A. Big effects from small changes: Possible ways to explore nature's chemical diversity. ChemBioChem 2002, 3, 619-627. [CrossRef]

176. Scherlach, K.; Hertweck, C. Triggering cryptic natural product biosynthesis in microorganisms. Org. Biomol. Chem. 2009, 7, 1753-1760. [CrossRef] [PubMed]

177. Oh, D.C.; Kauffman, C.A.; Jensen, P.R.; Fenical, W. Induced production of emericellamides A and B from the marine-derived fungus Emericella sp. in competing co-culture. J. Nat. Prod. 2007, 70, 515-520. [CrossRef] [PubMed]

178. Yeh, H.H.; Ahuja, M.; Chiang, Y.M.; Oakley, C.E.; Moore, S.; Yoon, O.; Hajovsky, H.; Bok, J.W.; Keller, N.P.; Wang, C.C.; et al. Resistance Gene-Guided Genome Mining: Serial Promoter Exchanges in Aspergillus nidulans Reveal the Biosynthetic Pathway for Fellutamide B, a Proteasome Inhibitor. ACS Chem. Biol. 2016, 11, 2275-2284. [CrossRef]

179. Williams, R.B.; Henrikson, J.C.; Hoover, A.R.; Lee, A.E.; Cichewicz, R.H. Epigenetic remodeling of the fungal secondary metabolome. Org. Biomol. Chem. 2008, 6, 1895-1897. [CrossRef] 